\title{
WELL POSEDNESS AND FINITE ELEMENT APPROXIMABILITY OF TWO-DIMENSIONAL TIME-HARMONIC ELECTROMAGNETIC PROBLEMS INVOLVING NON-CONDUCTING MOVING OBJECTS WITH STATIONARY BOUNDARIES
}

\author{
Massimo Brignone ${ }^{1}$ AND Mirco RAFFetto ${ }^{1}$
}

\begin{abstract}
A set of sufficient conditions for the well-posedness and the convergence of the finite element approximation of two-dimensional time-harmonic electromagnetic boundary value problems involving non-conducting moving objects with stationary boundaries is provided for the first time to the best of authors's knowledge. The set splits into two parts. The first of these is made up of traditional conditions, which are not restrictive for practical applications and define the usual requirements for the domain, its boundary, its subdomains and their boundaries, the boundary conditions and the constitutive parameters. The second part consists of conditions which are specific for the problems at hand. In particular, these conditions are expressed in terms of the constitutive parameters of the media involved and of the velocity field. It is shown that it is not difficult to check the validity of these conditions and that they hold true for broad classes of practically important problems which involve axially moving media.
\end{abstract}

Mathematics Subject Classification. 65N30, 65N12, 35Q60.

Received June 12, 2014. Revised November 17, 2014.

Published online June 30, 2015.

\section{INTRODUCTION}

Electromagnetic waves interact with moving bodies in many applications [1]. In order to study or simulate such an interaction one has usually to formulate problems in the time domain [1]. In some cases, however, frequency domain formulations are still possible $[1,2]$. This is the case, for example, when the movement takes place in such a way that the boundaries of the moving objects are stationary $[1,2]$.

This class of problems includes those involving axially moving cylinders, which are of particular interest for their important applications in nuclear and plasma physics, astrophysics, and engineering. Just to cite a few of these applications, one could be interested in problems involving axially moving plasma columns [3-5], ionized meteor trails [6], jet exhausts [7] or mass flows in pneumatic pipes [8].

\footnotetext{
Keywords and phrases. Electromagnetic scattering, time-harmonic electromagnetic fields, bianisotropic media, moving media, variational formulation, well posedness, finite element method, convergence of the approximation.

1 Department of Electrical, Electronic, Telecommunications Engineering and Naval Architecture, University of Genoa, Via Opera Pia 11a, 16145, Genoa, Italy. massimo.brignone@unige.it; raffetto@dibe.unige.it
} 
For the importance of these applications several series solutions have been deduced. This was possible for time-harmonic electromagnetic scattering problems involving axially moving cylinders of simple cross-sections like the half space $[9,10]$ or the homogeneous or multilayer circular or elliptical cylinder $[4,5,7,11]$.

In the presence of scatterers moving with a uniform axial speed in vacuum, the scattering problem can be easily managed by transforming the incident field as it is seen from the reference frame in which the scatterers are at rest, by solving the scattering problem with all matter at rest (the vacuum is at rest in any reference frame), and by transforming back the solution to the field in the frame of interest [4].

However, in most cases of practical interest, which could involve multiple cylinders having irregular shapes, constitutive parameters and velocity fields, no result seems to be available and numerical methods are required to try to approximate the solutions of interest [12].

Unfortunately, when media in motion are involved, even in the cases of interest in which the boundaries among different materials are stationary, the electromagnetic problems become quite complex. This is due to the fact that even an isotropic medium appears as a bianisotropic material in any reference frame in which the medium itself is not at rest $[2,13]$.

The available results on the well-posedness of time-harmonic electromagnetic boundary value problems involving moving media or bianisotropic materials and on their numerical approximability are very few. For example, in [14] we provide some of the indicated results on the well-posedness of the problems of interest and the convergence of Galerkin finite element approximations. However, these results are deduced by exploiting in a crucial way the losses of the media involved in the problem and do not provide, for example, any indication in the presence of a lossless medium. Unfortunately, in astrophysics or in plasma physics it is rather common to deal with models involving such media [4]. As it is clearly pointed out in the Introduction of that paper, at that time no other well-posedness result for bianisotropic materials could be found by the authors in the open literature. In the last years the well-posedness of time-harmonic problems in the presence of moving or bianisotropic materials has been tackled just in [15], to the best of the authors knowledge. However, in that paper the results are obtained by assuming hypotheses preventing the presence of dielectric interfaces and cannot be applied to many cases of interest for the present analysis. Finally, apart from the simple case indicated above of cylinders moving with a uniform axial speed in vacuum, we cannot simply deduce the results of well-posedness and finite element approximability for any specific problem of interest from what we know in the absence of motion, even when the values of the velocities are exceedingly small with respect to the velocity of light, as it is often the case in most problems [1]. This is because, on the one hand, even the smallest bianisotropic effect is sufficient to prevent, as far as the authors are aware, the application of all techniques exploited so far to prove well-posedness and convergence of finite element approximations in the presence of motionless anisotropic media. On the other hand, a perturbation technique [16] could easily guarantee that, for any specific configuration of the media in a given domain, there exists a sufficiently small upper bound for the magnitude of the axial speed such that the results of interest hold true, but from such an approach it is extremely difficult to deduce explicit bounds for the velocity field and, then, establish whether a specific problem of interest involving moving media is well posed or not.

Thus the usual approach to tackle the problems of interest and deduce some of their properties is to use numerical methods to approximate their solutions [12]. Unfortunately, as pointed out before, due to the lack of theoretical results, this is done without any guarantee on the existence and uniqueness of the solutions themselves and on the reliability of the numerical methods adopted.

For the indicated reasons, the aim of this work is to provide some results on the well-posedness and the convergence of the finite element approximation for two-dimensional time-harmonic electromagnetic boundary value problems involving non-conducting moving objects with stationary boundaries. In particular, these results will be proved if a given set of sufficient conditions, expressed in terms of the constitutive parameters of the media involved and of the velocity field, are satisfied. These conditions will be shown to enforce very weak limitations on the magnitude of the axial speeds of the media involved. Finally, it will be shown that, with very simple evaluations, anyone will be able to know in advance if a problem of interest is well posed and its solution can be approximated by a converging finite element method. 
The indicated results will be deduced by assuming, in addition to the traditional hypotheses on the domain, its boundary, its subdomains and their boundaries, the boundary conditions and the constitutive parameters, that

- all the media involved are linear, time-invariant and isotropic in their rest frames. In such frames they are characterized by their permittivity, permeability and electric conductivity. This assumption is considered because of the lack of results for the problems of interest even in the simplest situations; it allows, moreover, to avoid many technicalities;

- the velocity field is time-invariant. This assumption together with the linearity of the media and the timeinvariance of the media and the boundaries is considered in order to formulate the problem in the frequency domain [2];

- the velocity field is piecewise analytic. The permittivity and the permeability are piecewise analytic for all media in motion. On the one hand, since piecewise analyticity and not global analyticity is assumed, discontinuities of the constitutive parameters and of the velocity field could be present in our models. On the other hand, such a piecewise analyticity is required to exploit the results of [17] and prove a uniqueness result in the presence of moving (bianisotropic) media. However, any new result of unique continuation [18] (p. 92) requiring weaker assumptions than the indicated piecewise analyticity could be adopted and, in such a case, the deductions of this paper will immediately hold true under these less restrictive hypotheses;

- all media in motion have an electric conductivity equal to zero. We do not assume any restrictive hypothesis on media at rest. The essential reason behind this type of restriction is to avoid the difficulties related to the convective currents which could also become surface electric currents [19,20]. It is important to observe that, apart from the indicated effect on the convective currents, such an assumption does not simplify the problem we have to deal with, since it is well known that losses are of help in proving, for example, the well-posedness of the problems of interest [14,18,21,22]. Moreover, in the presence of conduction losses in the moving media one has usually to consider the no-slip condition for (lossy) viscous fluids [23]. Such a condition neglects the possibility of having any discontinuity of the velocity field and, ultimately, prevents the possibility of using pure two-dimensional models, like the ones we consider in this paper, because close to a boundary the velocity is orthogonal to the boundary itself and this means that, in addition to the axial velocity away from the boundaries, the transverse components of the velocity field are not equal to zero. Finally, this type of assumption has been considered in many contributions dealing with two-dimensional problems in the presence of axial movement $[4,5,11,12,24,25]$, with very few exceptions [3].

The paper is organized as follows. In Section 2 the class of problems of interest is defined and all hypotheses giving a meaning to their standard (i.e., based on Maxwell's equations) mathematical formulations are clearly pointed out. In Section 3 any problem of interest is recast as a variational formulation, which is shown to be equivalent to it. In Section 4 and its subsections sufficient conditions for the well posedness of the variational formulation are found. Under which additional conditions it is possible to prove the convergence of Galerkin and finite element approximations is discussed in Sections 5 and 6, respectively. Finally, before concluding the paper, some practical implications are presented in Section 7. It is shown, in particular, that our sufficient conditions hold true for broad classes of problems involving axially moving media.

\section{Problem DEFinition}

In this paper, we are interested in dealing with problems having a cylindrical geometry. $z$ will denote the axis of such a geometry. The time-harmonic sources and the inhomogeneous boundary conditions involved are independent of $z$, too, so that our problems can be formulated in a two-dimensional domain $\Omega$ contained in the $(x, y)$ plane.

$\Gamma$ denotes the boundary of $\Omega . \mathbf{n}$ and $\mathbf{l}$ are the unit vectors orthogonal (pointing outward) and tangential to $\Gamma$, respectively (see [26], p. 34). We have $\mathbf{n} \times \mathbf{l}=\hat{\mathbf{z}}$ ([26], p. 34). 
We assume that the domain and its boundary are such that:

HD1. $\Omega \subset \mathbb{R}^{2}$ is open, bounded and connected,

HD2. $\Gamma$ is Lipschitz continuous ([26], p. 4).

The media involved in our problems can move in the axial direction with respect to the chosen reference frame. In such a frame a velocity field $v_{z}$ is naturally defined, even though we will often refer to it in terms of the usual ([27], p. 525) real-valued normalized field $\beta=\frac{v_{z}}{c_{0}}$, being $c_{0}$ the speed of light in vacuum. It is assumed that

HV1. $\beta$ is time-invariant.

Different inhomogeneous materials will be modelled in this work. We assume that

HM1. any material involved is linear and time-invariant

Due to the linearity of all media involved (HM1) and the time-invariance of the material characteristics (HM1), of the moving object boundaries and of $\beta$ (HV1), all fields in all media will be time-harmonic, as the considered sources. A factor $\mathrm{e}^{j \omega t}$, common to all fields of interest, is assumed and suppressed.

In order to avoid many cumbersome developments, even though simple in principle, in the following we will consider

HM2. any material involved is isotropic in its rest frame and is there characterized by its relative permittivity $\varepsilon_{r}$, its relative permeability $\mu_{r}$ and its electric conductivity $\sigma$.

In the following, any reference to $\varepsilon_{r}, \mu_{r}$ or $\sigma$ of a moving medium should be interpreted as a reference to the corresponding quantity when the medium is at rest.

Since all moving objects have stationary boundaries, we can also assume, without loss of generality, that from a geometrical point of view

HD3. $\Omega$ can be decomposed into $m$ subdomains (non-empty, open and connected subsets of $\Omega$ having Lipschitz continuous stationary boundaries) denoted $\Omega_{i}, i \in M=\{1, \ldots, m\}$, satisfying $\bar{\Omega}=\bar{\Omega}_{1} \cup \cdots \cup \bar{\Omega}_{m}(\bar{\Omega}$ is the closure of $\Omega$ ) and $\Omega_{i} \cap \Omega_{j}=\emptyset$ for $i \neq j$.

The meaning of this splitting of the domain is clarified by the following additional assumptions on the velocity field and on the media involved

HV2. $\left.\beta\right|_{\Omega_{k}}, k \in M$, is a restriction of a real-valued function which is analytic in an open set $U_{k}$, with $\bar{\Omega}_{k} \subset U_{k}$. HV3. we can find $M_{0} \subset M, M_{0} \neq \emptyset$, such that $\left.\beta\right|_{\Omega_{k}}=0 \forall k \in M_{0} ; M_{\beta}=M \backslash M_{0}$ and $\left.\beta\right|_{\Omega_{k}}$ is not identically zero $\forall k \in M_{\beta}$,

Remark 2.1. When both HV2 and HV3 hold true one easily deduces that $\beta$ is almost everywhere different from zero in $\Omega_{k}$ for all $k \in M_{\beta}$.

HM3. $\left.\varepsilon_{r}\right|_{\Omega_{k}},\left.\mu_{r}\right|_{\Omega_{k}},\left.\sigma\right|_{\Omega_{k}} \in C^{2}\left(\bar{\Omega}_{k}\right) \forall k \in M_{0}$. Otherwise, $\left.\varepsilon_{r}\right|_{\Omega_{k}},\left.\mu_{r}\right|_{\Omega_{k}}$ and $\left.\sigma\right|_{\Omega_{k}}$ are restrictions of real-valued functions which are analytic in an open set $U_{k}$, with $\bar{\Omega}_{k} \subset U_{k}$.

HV3 is considered just to give a meaning to the selected reference frame. The other technical assumptions, HV2 and HM3, explain that the domain splitting is performed on the basis of regularity of the constitutive parameters and of the velocity field. In particular, it is important to observe that the quantities involved in HV2 and HM3 are just piecewise but not globally regular fields. The regularity required in HV2 and HM3 is necessary in order to use some results of unique continuation [18,22] or analytic continuation [17]. In particular, the parts of these hypotheses requiring the analyticity of the corresponding fields can be weakened as soon as a unique continuation result for the electromagnetic field in the presence of moving or bianisotropic media is available and the results of [17] are not anymore necessary. Finally, the constitutive parameters of moving media are considered to be real-valued as it is usually the case in this type of studies [4,28-30]. However, it is important to point out that all moving media will be perceived as bianisotropic media in the selected reference frame and this is the major difficulty to be overcome in this paper. 
Remark 2.2. For any given splitting of the domain satisfying HD3 and such that HV2, HV3 and HM3 are verified, we can define a finer decomposition of $\Omega$. As a matter of fact, it is sufficient to observe that any $\Omega_{i}$, $i \in M$, can be split into subdomains without affecting the validity of the indicated hypotheses. This consideration will be heavily exploited in Section 7, where a particular decomposition of the subdomains $\Omega_{i}$ for $i \in M_{\beta}$ will be considered.

Without some additional hypotheses on the constitutive parameters we would be forced to deal with convective currents which could become surface electric currents in the presence of discontinuities of the velocity field [19,20]. This is what could happen, for example, when a cylinder with finite conductivity moves in the axial direction with a uniform velocity and the background medium is considered to have a zero electric conductivity [3]. Other examples involving real media on both sides can be cited but the conclusions in these cases are more controversial $[23,31]$. For these reasons, in order to avoid these difficulties, in this paper we assume

HM4. $\left.\sigma\right|_{\Omega_{k}}=0$ if $\left.\beta\right|_{\Omega_{k}} \neq 0$.

The reader could observe that HM3 and HM4 imply that all moving media are lossless in their rest frames. We hope to be able to relax this constraint in a future work, so as to include lossy moving materials in our analysis. However, by considering that losses are usually of help in proving the well-posedness of the problem, in this paper we have to deal with the most difficult cases. In particular, we will deal with cases which cannot be managed by using the results provided in [14].

As usual for motionless media, we include the effects of the electric conductivity in their permittivity by defining an equivalent relative permittivity, still denoted by $\varepsilon_{r}$ and given by $\varepsilon_{r}-j \frac{\sigma}{\omega \varepsilon_{0}}$ ([1], p. 359). Thus, taking account of HM4, in the following we will have to deal just with permittivity and permeability (in the chosen frame for media at rest and in their rest frames for moving media).

As pointed out by Cheng and Kong [2], a medium satisfying HM1, HM2, HM3 and HM4, which is in motion with respect to the chosen reference frame according to HV1, can be studied as a stationary bianisotropic material and this fact is especially useful in solving problems involving moving media with stationary boundaries, as in the present work. In particular, by using the subscript " $t$ " to denote the field quantities transverse to the $z$ direction we deduce $[4,28,29]$ :

$$
\begin{gathered}
\mathbf{D}_{t}=\frac{1+\mu_{r} \varepsilon_{r}-\zeta_{1}}{c_{0}^{2} \mu_{0} \mu_{r}} \mathbf{E}_{t}+\frac{\zeta_{2}}{c_{0} \mu_{0} \mu_{r}} \hat{\mathbf{z}} \times \mathbf{B}_{t} \quad \text { (a.e.) in } \Omega, \\
D_{z}=\varepsilon_{0} \varepsilon_{r} E_{z} \quad \text { in } \Omega, \\
\mathbf{H}_{t}=\frac{\zeta_{1}}{\mu_{0} \mu_{r}} \mathbf{B}_{t}+\frac{\zeta_{2}}{c_{0} \mu_{0} \mu_{r}} \hat{\mathbf{z}} \times \mathbf{E}_{t} \quad \text { in } \Omega, \\
H_{z}=\frac{1}{\mu_{0} \mu_{r}} B_{z} \quad \text { in } \Omega,
\end{gathered}
$$

where $\varepsilon_{0}$ and $\mu_{0}$ are, respectively, the permittivity and permeability of vacuum and $[4,29]$ :

$$
\begin{gathered}
\zeta_{1}=\frac{1-\mu_{r} \varepsilon_{r} \beta^{2}}{1-\beta^{2}} \text { in } \Omega, \\
\zeta_{2}=\frac{\beta\left(\mu_{r} \varepsilon_{r}-1\right)}{1-\beta^{2}} \text { in } \Omega .
\end{gathered}
$$

In order to deal with bounded quantities we assume, in addition to HM3, the following additional hypothesis

HM5. we can find $C_{\mu m}>0$ such that $\left|\mu_{r}\right| \geq C_{\mu m}$ in $\Omega_{k}, \forall k \in M$.

HV4. we can find $C_{\beta M}: 0 \leq|\beta| \leq C_{\beta M}<1$ in $\Omega_{k}, \forall k \in M$. 
The latter is closely related, even though stronger, to one of the best known conclusions of the special theory of relativity. The deduced form of the constitutive relations is correct in $\Omega$, even where the involved media are motionless.

In order to define the problem of interest we introduce the following additional notations. Let $\left(L^{2}(\Omega)\right)^{n}$ be the usual Hilbert space of square integrable vector fields on $\Omega$ with values in $\mathbb{C}^{n}, n=2,3$, and with scalar product given by $(\mathbf{u}, \mathbf{v})_{0, \Omega}=\int_{\Omega} \mathbf{v}^{*} \mathbf{u} \mathrm{d} V$, where $\mathbf{v}^{*}$ denotes the conjugate transpose of the column vector $\mathbf{v}$. For a given three-dimensional complex-valued vector field $\mathbf{A}=\left(A_{x}, A_{y}, A_{z}\right) \in\left(L^{2}(\Omega)\right)^{3}$ we consider the operators $\operatorname{curl}_{2 D}$ and $\operatorname{grad}_{2 D}$, defined according to ([32], p. 201),

$$
\begin{aligned}
\operatorname{curl}_{2 D} \mathbf{A}_{t} & =\frac{\partial A_{y}}{\partial x}-\frac{\partial A_{x}}{\partial y}, \\
\operatorname{grad}_{2 D} A_{z} & =\left(\frac{\partial A_{z}}{\partial x}, \frac{\partial A_{z}}{\partial y}\right) .
\end{aligned}
$$

Assumptions HM1, HM2, HM3, HM4 and the hypotheses that will follow on the impressed sources do not allow the presence of surface electric or magnetic currents. For this reason, the Hilbert space where we will seek the transverse part of the electric and magnetic fields is [33]

$$
U_{2 D}=\left\{\mathbf{A}_{t} \in\left(L^{2}(\Omega)\right)^{2} \mid \operatorname{curl}_{2 D} \mathbf{A}_{t} \in L^{2}(\Omega) \text { and } \mathbf{A}_{t} \cdot \mathbf{l} \in L^{2}(\Gamma)\right\},
$$

where, for any $\mathbf{A}_{t} \in H\left(\operatorname{curl}_{2 D}, \Omega\right)=\left\{\mathbf{A}_{t} \in\left(L^{2}(\Omega)\right)^{2} \mid \operatorname{curl}_{2 D} \mathbf{A}_{t} \in L^{2}(\Omega)\right\}$, the meaning of the boundary values $\mathbf{A}_{t} \cdot \mathbf{l}$ is defined in ([26], p. 34).

The inner product in this space is given by

$$
\left(\mathbf{u}_{t}, \mathbf{v}_{t}\right)_{U_{2 D}}=\left(\mathbf{u}_{t}, \mathbf{v}_{t}\right)_{0, \Omega}+\left(\operatorname{curl}_{2 D} \mathbf{u}_{t}, \operatorname{curl}_{2 D} \mathbf{v}_{t}\right)_{0, \Omega}+\left(\mathbf{u}_{t} \cdot \mathbf{l}, \mathbf{v}_{t} \cdot \mathbf{l}\right)_{0, \Gamma}
$$

The axial components of the same fields will be in the Hilbert space $H^{1}(\Omega)$ ([26], p. 2) for the same reasons. For any axial component $A_{z} \in H^{1}(\Omega)$ we denote by $\gamma_{0} A_{z}$ its boundary values ([26], p. 8). $(,)_{1, \Omega}$ will indicate the usual inner product in this space.

In order to shorten many formulas we define also

$$
U=U_{2 D} \times H^{1}(\Omega)
$$

which is a Hilbert space with inner product given by

$$
(\mathbf{u}, \mathbf{v})_{U}=\left(\mathbf{u}_{t}, \mathbf{v}_{t}\right)_{U_{2 D}}+\left(u_{z}, v_{z}\right)_{1, \Omega}
$$

\| $\|_{U}$ will denote the corresponding norm.

The symbol $\omega$ represents the angular frequency. Without loss of generality for the problems of interest, we assume that it is real and positive. Moreover, $\mathbf{J}_{e}, \mathbf{J}_{m} \in\left(L^{2}(\Omega)\right)^{3}$ are the electric and magnetic current densities, respectively, prescribed by the sources. We have already pointed out that the currents induced by the field could be present just in motionless media and are taken into account in the displacement field $\mathbf{D}$.

$Y$ will be the scalar complex admittance involved in impedance boundary conditions; $f_{R l}$ and $f_{R z}$ will be the corresponding inhomogeneous terms. Finally, the complex-valued admittance function $Y$ with domain $\Gamma$ is assumed to satisfy

HB1. $Y$ is piecewise continuous and $|Y|$ is bounded.

We are now in a position to state the electromagnetic boundary value problem we will address in this paper. 
Problem 1. Under the hypotheses HD1-HD3, HV1-HV4, HM1-HM5, HB1, given $\omega>0, \mathbf{J}_{e}, \mathbf{J}_{m} \in\left(L^{2}(\Omega)\right)^{3}$ and $f_{R l}, f_{R z} \in L^{2}(\Gamma)$, find $(\mathbf{E}, \mathbf{B}, \mathbf{H}, \mathbf{D}) \in U \times\left(L^{2}(\Omega)\right)^{3} \times U \times\left(L^{2}(\Omega)\right)^{3}$ which satisfies the following system of Maxwell's equations and impedance boundary conditions

$$
\begin{cases}\operatorname{curl}_{2 D} \mathbf{H}_{t}-j \omega D_{z}=J_{e z} & \text { in } \Omega \\ \operatorname{grad}_{2 D} H_{z} \times \hat{\mathbf{z}}-j \omega \mathbf{D}_{t}=\mathbf{J}_{e t} & \text { in } \Omega \\ \operatorname{curl}_{2 D} \mathbf{E}_{t}+j \omega B_{z}=-J_{m z} & \text { in } \Omega \\ \operatorname{grad}_{2 D} E_{z} \times \hat{\mathbf{z}}+j \omega \mathbf{B}_{t}=-\mathbf{J}_{m t} & \text { in } \Omega \\ \mathbf{H}_{t} \cdot \mathbf{l}+Y\left(\gamma_{0} E_{z}\right)=-f_{R z} & \text { on } \Gamma \\ \gamma_{0} H_{z}-Y\left(\mathbf{E}_{t} \cdot \mathbf{l}\right)=f_{R l} & \text { on } \Gamma\end{cases}
$$

and the constitutive relations $(2.1)-(2.4)$.

The reader should observe that equations $(2.13)_{i}, i=5,6$, with hypothesis HB1 can be used, on the one hand, to enforce lowest order absorbing boundary conditions ([34], p. 9), so that the above model can be thought of as an approximation of a two-dimensional radiation or scattering problem involving objects moving in the direction orthogonal to the domain. On the other hand, they can enforce boundary conditions at imperfectly conducting surfaces ([35], pp. 384-385), so that the model of interest can be thought of as a realistic formulation of a two-dimensional cavity problem involving again moving objects with stationary boundaries.

More complex boundary conditions could be considered as well. However, we chose the above simple model since, on the one hand, the generality of our results is not reduced in a significant way and, on the other hand, the mathematical developments can be limited to a reasonable extent.

\section{VARIATIONAL FORMULATION}

One of the targets of this contribution is to discuss under which conditions it is possible to prove the wellposedness of Problem 1. In order to obtain such a result it is useful to deduce from Problem 1 a variational formulation. This will also be of help to obtain another result: the convergence of Galerkin and finite element approximations.

A variational finite element formulation of the problem of interest can be easily obtained by following the developments reported in Section 3 of [14]. We report here the main steps in order to ease the reader and to introduce many notations that will be heavily exploited later on.

In particular, in analogy to Lemma 1 of [14], we have

Lemma 3.1. Any solution of Problem 1 is fully determined by its $\mathbf{E}$ component through equation (2.2) and the following relations

$$
\begin{gathered}
B_{z}=-\frac{1}{j \omega} J_{m z}-\frac{1}{j \omega} \operatorname{curl}_{2 D} \mathbf{E}_{t}, \\
\mathbf{B}_{t}=-\frac{1}{j \omega} \mathbf{J}_{m t}-\frac{1}{j \omega} \operatorname{grad}_{2 D} E_{z} \times \hat{\mathbf{z}}, \\
H_{z}=-\frac{1}{j \omega \mu_{0} \mu_{r}} J_{m z}-\frac{1}{j \omega \mu_{0} \mu_{r}} \operatorname{curl}_{2 D} \mathbf{E}_{t}, \\
\mathbf{H}_{t}=\frac{\zeta_{2}}{c_{0} \mu_{0} \mu_{r}} \hat{\mathbf{z}} \times \mathbf{E}_{t}-\frac{\zeta_{1}}{j \omega \mu_{0} \mu_{r}} \mathbf{J}_{m t}-\frac{\zeta_{1}}{j \omega \mu_{0} \mu_{r}} \operatorname{grad}_{2 D} E_{z} \times \hat{\mathbf{z}}, \\
\mathbf{D}_{t}=\frac{1+\mu_{r} \varepsilon_{r}-\zeta_{1}}{c_{0}^{2} \mu_{0} \mu_{r}} \mathbf{E}_{t}-\frac{\zeta_{2}}{j \omega c_{0} \mu_{0} \mu_{r}} \hat{\mathbf{z}} \times \mathbf{J}_{m t}-\frac{\zeta_{2}}{j \omega c_{0} \mu_{0} \mu_{r}} \operatorname{grad}_{2 D} E_{z} .
\end{gathered}
$$


Proof. Suppose (E, B, H, D) satisfies Problem 1. Then, as $\omega>0$, from (2.13) 3 we deduce (3.1) and from $(2.13)_{4}$ we deduce (3.2). $\frac{1}{\mu_{r}}$ is bounded by HM5. Thus, from (2.4) and (3.1) we deduce (3.3). Analogously, from (2.3) and (3.2) we deduce (3.4). Finally, we obtain (3.5) from (2.1) and (3.4). Note that $D_{z}$ is directly given in terms of $E_{z}$ by (2.2).

Now, according to [14] we take the $L^{2}$ scalar products of $(2.13)_{1}$ with any field $w_{z} \in H^{1}(\Omega)$ and of $(2.13)_{2}$ with any vector field $\mathbf{w}_{t} \in U_{2 D}$. We get

$$
\begin{cases}\left(\operatorname{curl}_{2 D} \mathbf{H}_{t}-j \omega D_{z}, w_{z}\right)_{0, \Omega}=\left(J_{e z}, w_{z}\right)_{0, \Omega} & \forall w_{z} \in H^{1}(\Omega) \\ \left(\operatorname{grad}_{2 D} H_{z} \times \hat{\mathbf{z}}-j \omega \mathbf{D}_{t}, \mathbf{w}_{t}\right)_{0, \Omega}=\left(\mathbf{J}_{e t}, \mathbf{w}_{t}\right)_{0, \Omega} & \forall \mathbf{w}_{t} \in U_{2 D}\end{cases}
$$

Taking account of HD1 and HD2 we can manipulate the above formulas. In particular, $\operatorname{since} \operatorname{curl}_{2 D} \mathbf{u}_{t}=$ $-\operatorname{div}_{2 D}\left(\hat{\mathbf{z}} \times \mathbf{u}_{t}\right)$ for all $\mathbf{u}_{t} \in\left(L^{2}(\Omega)\right)^{2}$ ([32], p. 205), by using (1.10) of ([32], p. 206) and the additional regularity of the boundary values of fields in $U_{2 D}$ we obtain

$$
\left(\operatorname{curl}_{2 D} \mathbf{H}_{t}, w_{z}\right)_{0, \Omega}=\left(\hat{\mathbf{z}} \times \mathbf{H}_{t}, \operatorname{grad}_{2 D} w_{z}\right)_{0, \Omega}+\left(\mathbf{H}_{t} \cdot \mathbf{l}, \gamma_{0} w_{z}\right)_{0, \Gamma} \quad \forall w_{z} \in H^{1}(\Omega) .
$$

Moreover, since $\left(\operatorname{grad}_{2 D} H_{z} \times \hat{\mathbf{z}}, \mathbf{w}_{t}\right)_{0, \Omega}=\left(\operatorname{grad}_{2 D} H_{z}, \hat{\mathbf{z}} \times \mathbf{w}_{t}\right)_{0, \Omega}$, by using again (1.10) of ([32], p. 206) and the additional regularity of the boundary values of fields in $U_{2 D}$ we deduce

$$
\left(\operatorname{grad}_{2 D} H_{z} \times \hat{\mathbf{z}}, \mathbf{w}_{t}\right)_{0, \Omega}=\left(H_{z}, \operatorname{curl}_{2 D} \mathbf{w}_{t}\right)_{0, \Omega}-\left(\gamma_{0} H_{z}, \mathbf{w}_{t} \cdot \mathbf{l}\right)_{0, \Gamma} \quad \forall \mathbf{w}_{t} \in U_{2 D}
$$

By substituting (3.7) and (3.8) in (3.6) we get

$$
\begin{cases}\left(\hat{\mathbf{z}} \times \mathbf{H}_{t}, \operatorname{grad}_{2 D} w_{z}\right)_{0, \Omega}-j \omega\left(D_{z}, w_{z}\right)_{0, \Omega}+\left(\mathbf{H}_{t} \cdot \mathbf{l}, \gamma_{0} w_{z}\right)_{0, \Gamma}=\left(J_{e z}, w_{z}\right)_{0, \Omega} & \forall w_{z} \in H^{1}(\Omega) \\ \left(H_{z}, \operatorname{curl}_{2 D} \mathbf{w}_{t}\right)_{0, \Omega}-j \omega\left(\mathbf{D}_{t}, \mathbf{w}_{t}\right)_{0, \Omega}-\left(\gamma_{0} H_{z}, \mathbf{w}_{t} \cdot \mathbf{l}\right)_{0, \Gamma}=\left(\mathbf{J}_{e t}, \mathbf{w}_{t}\right)_{0, \Omega} & \forall \mathbf{w}_{t} \in U_{2 D} .\end{cases}
$$

Let us now substitute (3.4), (2.2) and (2.13) $)_{5}$ in equation $(3.9)_{1}$ and multiply it by $-j \omega \mu_{0}$. Analogously, let us substitute $(3.3),(3.5)$ and $(2.13)_{6}$ in equation $(3.9)_{2}$ and multiply it by $-j \omega \mu_{0}$. We obtain:

$$
\left\{\begin{aligned}
\left(\frac{\zeta_{1}}{\mu_{r}}\right. & \left.\operatorname{grad}_{2 D} E_{z}, \operatorname{grad}_{2 D} w_{z}\right)_{0, \Omega}-\frac{\omega^{2}}{c_{0}^{2}}\left(\varepsilon_{r} E_{z}, w_{z}\right)_{0, \Omega} \\
& +j \frac{\omega}{c_{0}}\left(\frac{\zeta_{2}}{\mu_{r}} \mathbf{E}_{t}, \operatorname{grad}_{2 D} w_{z}\right)_{0, \Omega}+j \omega \mu_{0}\left(Y\left(\gamma_{0} E_{z}\right), \gamma_{0} w_{z}\right)_{0, \Gamma} \\
& =-j \omega \mu_{0}\left(J_{e z}, w_{z}\right)_{0, \Omega}-\left(\frac{\zeta_{1}}{\mu_{r}} \hat{\mathbf{z}} \times \mathbf{J}_{m t}, \operatorname{grad}_{2 D} w_{z}\right)_{0, \Omega} \\
& -j \omega \mu_{0}\left(f_{R z}, \gamma_{0} w_{z}\right)_{0, \Gamma} \\
\left(\frac{1}{\mu_{r}}\right. & \left.\operatorname{curl}_{2 D} \mathbf{E}_{t}, \operatorname{curl}_{2 D} \mathbf{w}_{t}\right)_{0, \Omega}-\frac{\omega^{2}}{c_{0}^{2}}\left(\frac{1+\varepsilon_{r} \mu_{r}-\zeta_{1}}{\mu_{r}} \mathbf{E}_{t}, \mathbf{w}_{t}\right)_{0, \Omega} \\
& -j \frac{\omega}{c_{0}}\left(\frac{\zeta_{2}}{\mu_{r}} \operatorname{grad}_{2 D} E_{z}, \mathbf{w}_{t}\right)_{0, \Omega}+j \omega \mu_{0}\left(Y\left(\mathbf{E}_{t} \cdot \mathbf{l}\right), \mathbf{w}_{t} \cdot \mathbf{l}\right)_{0, \Gamma}(\Omega) \\
& =-\left(\frac{1}{\mu_{r}} J_{m z}, \operatorname{curl}_{2 D} \mathbf{w}_{t}\right)_{0, \Omega}-j \omega \mu_{0}\left(\mathbf{J}_{e t}, \mathbf{w}_{t}\right)_{0, \Omega} \\
& +j \frac{\omega}{c_{0}}\left(\frac{\zeta_{2}}{\mu_{r}} \hat{\mathbf{z}} \times \mathbf{J}_{m t}, \mathbf{w}_{t}\right)_{0, \Omega}-j \omega \mu_{0}\left(f_{R l}, \mathbf{w}_{t} \cdot \mathbf{l}\right)_{0, \Gamma} \quad \forall \mathbf{w}_{t} \in U_{2 D},
\end{aligned}\right.
$$

where, taking account of HM3, HM5, HB1 and HV4, all terms have a meaning. 
Thus, by defining the sesquilinear form

$$
\begin{aligned}
a(\mathbf{u}, \mathbf{w})= & \left(\frac{\zeta_{1}}{\mu_{r}} \operatorname{grad}_{2 D} u_{z}, \operatorname{grad}_{2 D} w_{z}\right)_{0, \Omega}+\left(\frac{1}{\mu_{r}} \operatorname{curl}_{2 D} \mathbf{u}_{t}, \operatorname{curl}_{2 D} \mathbf{w}_{t}\right)_{0, \Omega} \\
& +j \frac{\omega}{c_{0}}\left(\frac{\zeta_{2}}{\mu_{r}} \mathbf{u}_{t}, \operatorname{grad}_{2 D} w_{z}\right)_{0, \Omega}-j \frac{\omega}{c_{0}}\left(\frac{\zeta_{2}}{\mu_{r}} \operatorname{grad}_{2 D} u_{z}, \mathbf{w}_{t}\right)_{0, \Omega} \\
& -\frac{\omega^{2}}{c_{0}^{2}}\left(\varepsilon_{r} u_{z}, w_{z}\right)_{0, \Omega}-\frac{\omega^{2}}{c_{0}^{2}}\left(\frac{1+\varepsilon_{r} \mu_{r}-\zeta_{1}}{\mu_{r}} \mathbf{u}_{t}, \mathbf{w}_{t}\right)_{0, \Omega} \\
& +j \omega \mu_{0}\left(Y\left(\gamma_{0} u_{z}\right), \gamma_{0} w_{z}\right)_{0, \Gamma}+j \omega \mu_{0}\left(Y\left(\mathbf{u}_{t} \cdot \mathbf{l}\right), \mathbf{w}_{t} \cdot \mathbf{l}\right)_{0, \Gamma}
\end{aligned}
$$

for all $\mathbf{u}, \mathbf{w} \in U$ and the antilinear form

$$
\begin{aligned}
l(\mathbf{w})= & -j \omega \mu_{0}\left(J_{e z}, w_{z}\right)_{0, \Omega}-\left(\frac{\zeta_{1}}{\mu_{r}} \hat{\mathbf{z}} \times \mathbf{J}_{m t}, \operatorname{grad}_{2 D} w_{z}\right)_{0, \Omega}-j \omega \mu_{0}\left(f_{R z}, \gamma_{0} w_{z}\right)_{0, \Gamma} \\
& -\left(\frac{1}{\mu_{r}} J_{m z}, \operatorname{curl}_{2 D} \mathbf{w}_{t}\right)_{0, \Omega}-j \omega \mu_{0}\left(\mathbf{J}_{e t}, \mathbf{w}_{t}\right)_{0, \Omega}+j \frac{\omega}{c_{0}}\left(\frac{\zeta_{2}}{\mu_{r}} \hat{\mathbf{z}} \times \mathbf{J}_{m t}, \mathbf{w}_{t}\right)_{0, \Omega} \\
& -j \omega \mu_{0}\left(f_{R l}, \mathbf{w}_{t} \cdot \mathbf{l}\right)_{0, \Gamma}
\end{aligned}
$$

for all $\mathbf{w} \in U$, we deduce the variational formulation we were looking for

Problem 2. Under the hypotheses HD1-HD3, HV1-HV4, HM1-HM5, HB1, given $\omega>0, \mathbf{J}_{e}, \mathbf{J}_{m} \in\left(L^{2}(\Omega)\right)^{3}$ and $f_{R l}, f_{R z} \in L^{2}(\Gamma)$, find $\mathbf{E} \in U$ such that

$$
a(\mathbf{E}, \mathbf{w})=l(\mathbf{w}) \quad \forall \mathbf{w} \in U .
$$

By using HM3, HM5, HB1 and HV4 the reader can easily check that the sesquilinear form $a$ and the antilinear form $l$ are bounded.

In deducing Problem 2 from Problem 1, we have shown that the $\mathbf{E}$ component of any solution of Problem 1 satisfies Problem 2.

Vice versa, as shown in details in [14], we can prove that any solution of Problem 2 is the $\mathbf{E}$ component of a solution of Problem 1, the other components being given by (3.1)-(3.5) and (2.2). These details are skipped because, on the one hand, they can be easily deduced from [14], and, on the other hand, all notations of interest have already been introduced.

We simply recall from [14] two important results for our analysis. Their deductions would be trivial because all considerations related to these points and reported in [14] exactly apply in this case, too.

Theorem 3.2. If $(\mathbf{E}, \mathbf{B}, \mathbf{H}, \mathbf{D})$ satisfies Problem 1, then $\mathbf{E}$ satisfies Problem 2. Conversely, if $\mathbf{E}$ satisfies Problem 2 and $\mathbf{B}, \mathbf{H}$ and $\mathbf{D}$ are obtained by (3.1)-(3.5) and (2.2), then $(\mathbf{E}, \mathbf{B}, \mathbf{H}, \mathbf{D})$ satisfies Problem 1.

Theorem 3.3. Problem 1 is well-posed if and only if Problem 2 is well-posed.

\section{WELL POSEDNESS OF THE PROBLEM}

In this Section, by using the generalized Lax-Milgram lemma ([18], p. 21), we prove the well posedness of Problem 2 and, by Theorem 3.3, the well posedness of Problem 1. In order to achieve this result, different properties have to be deduced. Some of these deductions are long and, for this reason, we split this section into some subsections. The first two of these deal with uniqueness. The third is related to the inf-sup condition ([18], p. 21). 


\subsection{Uniqueness of the solution of the problem}

Since Problem 2 is linear, uniqueness can be addressed by showing that the homogeneous version of Problem 2 (i.e., the one with $l=0$ in Eq. (3.13)) admits only the solution $\mathbf{E}=0$ ([36], p. 20, [18], p. 92).

In order to obtain the result of interest in this subsection some additional assumptions should be considered. It is very well-known, for example, that the presence of some losses is crucial in order to obtain a uniqueness result ([18], p. 92). As a matter of fact, if no losses are present in the model of interest uniqueness cannot be guaranteed in general, even for simple problems involving a linear, homogeneous, passive and motionless medium.

In our problem, losses can be modelled in the boundary or in the media. We require in any case that the boundary and the media do not provide active power, that is

HM6. $\operatorname{Im}\left(\varepsilon_{r}\right) \leq 0$ and $\operatorname{Im}\left(\mu_{r}\right) \leq 0$ in $\Omega_{k}, \forall k \in M$

HB2. $\operatorname{Re}(Y) \geq 0$ on $\Gamma$.

To take account of some losses in the media we consider [37]

HM7. we can find $C_{e m M}>0, j \in M_{0}$ and $D \subset \Omega_{j}, D$ open non-empty, such that $\operatorname{Im}\left(\varepsilon_{r}\right) \leq-C_{e m M}$ or $\operatorname{Im}\left(\mu_{r}\right) \leq-C_{e m M}$ in $D$,

where Im denotes the imaginary part. This hypothesis refers to polarization or conduction losses in motionless media.

The other type of losses we could deal with is considered by:

HB3. we can find $C_{Y m}>0$ and a non-empty open part $\Gamma_{l}$ of $\Gamma$ such that $\operatorname{Re}(Y) \geq C_{Y m}$ almost everywhere on $\Gamma_{l}$,

where Re denotes the real part.

These hypotheses can be exploited to achieve a first intermediate result. Its deduction is easily got by using the procedure defined in [22]. We will provide all details because we are in the presence of moving media and we have to take account of a sesquilinear form having more terms than usual.

In addition to the previous assumptions, in order to complete the deduction of interest for motionless media, we consider:

HM8. we can find $C_{\varepsilon m}>0$ such that $\left|\varepsilon_{r}\right| \geq C_{\varepsilon m}$ in $\Omega_{k}, \forall k \in M$.

Finally, the next two hypotheses, expressed in terms of the constitutive parameters of the media involved and of the velocity field, will be exploited to get another intermediate deduction related to a unique continuation result in the presence of moving media:

HMV1. the fields $\mu_{r}, \varepsilon_{r}$ and $\beta$ allow to find $C_{m v 1}>0$ such that $\left|\mu_{r} \varepsilon_{r}-\beta^{2}\right| \geq C_{m v 1}$ in $\Omega_{i}, \forall i \in M$.

Remark 4.1. HMV1 could be written as HMV1i for any specific $\Omega_{i}$ of interest. However, before obtaining the final uniqueness result we have to ask that it holds true for all $i \in M_{\beta}$. HM5 and HM8 directly imply the validity of HMV1 in the subdomains where $\beta=0$.

HMV2. the fields $\mu_{r}, \varepsilon_{r}$ and $\beta$ are such that

$$
\begin{aligned}
16 c_{0}^{2} \max _{\mathbf{x} \in \bar{\Omega}_{i}}\left(\frac{\beta^{2}\left(\mu_{r} \varepsilon_{r}-1\right)^{2}}{\left(\mu_{r} \varepsilon_{r}-\beta^{2}\right)^{2}}\right) & \\
& \quad<\left(-C_{\kappa, s, i}+\sqrt{C_{\kappa, s, i}^{2}+4 C_{\kappa, d, i} C_{\kappa, r, i}}\right)\left(-C_{\nu, s, i}+\sqrt{C_{\nu, s, i}^{2}+4 C_{\nu, d, i} C_{\nu, r, i}}\right)
\end{aligned}
$$


$\forall i \in M_{\beta}$, where

$$
\begin{gathered}
C_{\kappa, s, i}=\frac{1}{\varepsilon_{0}} \max _{\mathbf{x} \in \bar{\Omega}_{i}}\left(\max \left(2\left|\frac{\mu_{r}\left(1-\beta^{2}\right)}{\mu_{r} \varepsilon_{r}-\beta^{2}}\right|,\left|\frac{\mu_{r}\left(1-\beta^{2}\right)}{\mu_{r} \varepsilon_{r}-\beta^{2}}\right|+\frac{1}{\left|\varepsilon_{r}\right|}\right)\right), \\
C_{\nu, s, i}=\frac{1}{\mu_{0}} \max _{\mathbf{x} \in \bar{\Omega}_{i}}\left(\max \left(2\left|\frac{\varepsilon_{r}\left(1-\beta^{2}\right)}{\mu_{r} \varepsilon_{r}-\beta^{2}}\right|,\left|\frac{\varepsilon_{r}\left(1-\beta^{2}\right)}{\mu_{r} \varepsilon_{r}-\beta^{2}}\right|+\frac{1}{\left|\mu_{r}\right|}\right)\right), \\
C_{\kappa, d, i}=\min _{\mathbf{x} \in \bar{\Omega}_{i}}\left(\frac{\mu_{r}^{2}\left(1-\beta^{2}\right)^{2}}{\varepsilon_{0}^{3}\left|\varepsilon_{r}\right|\left(\mu_{r} \varepsilon_{r}-\beta^{2}\right)^{2}}\right), \\
C_{\nu, d, i}=\min _{\mathbf{x} \in \bar{\Omega}_{i}}\left(\frac{\varepsilon_{r}^{2}\left(1-\beta^{2}\right)^{2}}{\mu_{0}^{3}\left|\mu_{r}\right|\left(\mu_{r} \varepsilon_{r}-\beta^{2}\right)^{2}}\right) \\
C_{\nu, r, i}=\varepsilon_{0} \min \left(\min _{\mathbf{x} \in \bar{\Omega}_{i}}\left|\frac{\mu_{r} \varepsilon_{r}-\beta^{2}}{\mu_{r}\left(1-\beta^{2}\right)}\right|, \min _{\mathbf{x} \in \bar{\Omega}_{i}}\left|\varepsilon_{r}\right|\right) \\
\min \left(\min _{\mathbf{x} \in \bar{\Omega}_{i}}\left|\frac{\mu_{r} \varepsilon_{r}-\beta^{2}}{\varepsilon_{r}\left(1-\beta^{2}\right)}\right|, \min _{\mathbf{x} \in \bar{\Omega}_{i}}\left|\mu_{r}\right|\right) .
\end{gathered}
$$

Remark 4.2. Hypotheses HM5, HM8 and HV4 imply $C_{\kappa, d, i}>0$ and $C_{\nu, d, i}>0$. Analogously, hypotheses HM5, HM8 and HMV1 guarantee that $C_{\kappa, r, i}>0$ and $C_{\nu, r, i}>0$. Then the right-hand side of (4.1) is larger than zero. Thus, HMV2 is trivially satisfied in the subdomains where $\beta=0$ and we could consider it for all $i \in M$.

The main result of this subsection is given by:

Theorem 4.3. Under the hypotheses HD1-HD3, HV1-HV4, HM1-HM6, HM8, HB1-HB2, HMV1 and HMV2, Problem 2 admits a unique solution provided that at least one of HM7 and HB3 is satisfied.

In order to prove such a theorem the above indicated intermediate results are necessary. They are deduced with the following lemmas.

Lemma 4.4. Any solution $\mathbf{E}$ of Problem 2 with $l=0$ satisfies

- $\gamma_{0} E_{z}=0$ and $\mathbf{E}_{t} \cdot \mathbf{l}=0$ on $\Gamma_{l}$ if HM6, HB2 and HB3 hold true,

- $E_{z}=0, \operatorname{grad}_{2 D} E_{z}=0$ and $\mathbf{E}_{t}=0$ in $D$ if HM6, HB2 and the part relative to $\varepsilon_{r}$ of HM7 hold true,

- $\operatorname{grad}_{2 D} E_{z}=0, \mathbf{E}_{t}=0$ and $\operatorname{curl}_{2 D} \mathbf{E}_{t}=0$ in $D$ if HM6, HB2 and the part relative to $\mu_{r}$ of HM7 hold true.

Proof. Let us consider a solution $\mathbf{E}$ of the homogeneous problem. By choosing $\mathbf{w}=\mathbf{E}$ we deduce $a(\mathbf{E}, \mathbf{E})=0$ and, then, $\operatorname{Im}(a(\mathbf{E}, \mathbf{E}))=0$. But from equation (3.11) we deduce

$$
\begin{aligned}
0= & \operatorname{Im}(a(\mathbf{E}, \mathbf{E}))=\left(\operatorname{Im}\left(\frac{\zeta_{1}}{\mu_{r}}\right) \operatorname{grad}_{2 D} E_{z}, \operatorname{grad}_{2 D} E_{z}\right)_{0, \Omega} \\
& +\left(\operatorname{Im}\left(\frac{1}{\mu_{r}}\right) \operatorname{curl}_{2 D} \mathbf{E}_{t}, \operatorname{curl}_{2 D} \mathbf{E}_{t}\right)_{0, \Omega} \\
& +\frac{\omega}{c_{0}} \operatorname{Re}\left(\left(\frac{\zeta_{2}}{\mu_{r}} \mathbf{E}_{t}, \operatorname{grad}_{2 D} E_{z}\right)_{0, \Omega}-\left(\frac{\zeta_{2}}{\mu_{r}} \operatorname{grad}_{2 D} E_{z}, \mathbf{E}_{t}\right)_{0, \Omega}\right) \\
& -\frac{\omega^{2}}{c_{0}^{2}}\left(\operatorname{Im}\left(\varepsilon_{r}\right) E_{z}, E_{z}\right)_{0, \Omega}-\frac{\omega^{2}}{c_{0}^{2}}\left(\operatorname{Im}\left(\frac{1+\varepsilon_{r} \mu_{r}-\zeta_{1}}{\mu_{r}}\right) \mathbf{E}_{t}, \mathbf{E}_{t}\right)_{0, \Omega} \\
& +\omega \mu_{0}\left(\operatorname{Re}(Y)\left(\gamma_{0} E_{z}\right), \gamma_{0} E_{z}\right)_{0, \Gamma}+\omega \mu_{0}\left(\operatorname{Re}(Y)\left(\mathbf{E}_{t} \cdot \mathbf{l}\right), \mathbf{E}_{t} \cdot \mathbf{l}\right)_{0, \Gamma} .
\end{aligned}
$$


Taking into account that $\omega>0$ we easily see that the last two addends of the right-hand side member of (4.8) are not negative whenever HB2 holds true and could be strictly positive if $\gamma_{0} E_{z} \neq 0$ or $\mathbf{E}_{t} \cdot \mathbf{l} \neq 0$ on $\Gamma_{l}$ and HB3 is satisfied. The addend involving $\operatorname{Im}\left(\varepsilon_{r}\right)$ is not negative by HM6. It is strictly positive whenever HM6 and the part related to $\varepsilon_{r}$ of HM7 hold true, if $E_{z} \neq 0$ in $D$. The same result is deduced for the addend involving $\operatorname{Im}\left(\frac{1}{\mu_{r}}\right)$ because of the change of the sign of the imaginary part of $\frac{1}{\mu_{r}}$ with respect to that of $\mu_{r}$. In particular, the addend involving $\operatorname{Im}\left(\frac{1}{\mu_{r}}\right)$ is not negative by HM6 and is strictly positive whenever HM6 and the part related to $\mu_{r}$ of HM7 hold true, if $\operatorname{curl}_{2 D} \mathbf{E}_{t} \neq 0$ in $D$. In order to manage the remaining four addends of the right-hand side member of (4.8), let us firstly observe that, by using (2.5), we can deduce

$$
\frac{\zeta_{1}}{\mu_{r}}=\frac{1-\mu_{r} \varepsilon_{r} \beta^{2}}{\mu_{r}\left(1-\beta^{2}\right)}=\frac{1}{\mu_{r}} \frac{1}{1-\beta^{2}}-\varepsilon_{r} \frac{\beta^{2}}{1-\beta^{2}}
$$

and

$$
\frac{1+\varepsilon_{r} \mu_{r}-\zeta_{1}}{\mu_{r}}=\frac{1}{\mu_{r}}+\varepsilon_{r}-\frac{\zeta_{1}}{\mu_{r}}=\frac{1}{\mu_{r}}\left(1-\frac{1}{1-\beta^{2}}\right)+\varepsilon_{r}\left(1+\frac{\beta^{2}}{1-\beta^{2}}\right)=-\frac{1}{\mu_{r}} \frac{\beta^{2}}{1-\beta^{2}}+\varepsilon_{r} \frac{1}{1-\beta^{2}} .
$$

Moreover, by using (2.6) we obtain

$$
\frac{\zeta_{2}}{\mu_{r}}=\frac{\beta\left(\mu_{r} \varepsilon_{r}-1\right)}{\mu_{r}\left(1-\beta^{2}\right)}=-\frac{1}{\mu_{r}} \frac{\beta}{1-\beta^{2}}+\varepsilon_{r} \frac{\beta}{1-\beta^{2}} .
$$

Thus,

$$
\begin{aligned}
& \left(\frac{\zeta_{1}}{\mu_{r}} \operatorname{grad}_{2 D} E_{z}, \operatorname{grad}_{2 D} E_{z}\right)_{0, \Omega} \\
& +j \frac{\omega}{c_{0}}\left(\left(\frac{\zeta_{2}}{\mu_{r}} \mathbf{E}_{t}, \operatorname{grad}_{2 D} E_{z}\right)_{0, \Omega}-\left(\frac{\zeta_{2}}{\mu_{r}} \operatorname{grad}_{2 D} E_{z}, \mathbf{E}_{t}\right)_{0, \Omega}\right) \\
& -\frac{\omega^{2}}{c_{0}^{2}}\left(\frac{1+\varepsilon_{r} \mu_{r}-\zeta_{1}}{\mu_{r}} \mathbf{E}_{t}, \mathbf{E}_{t}\right)_{0, \Omega} \\
& =\int_{\Omega} \frac{1}{\mu_{r}} \frac{1}{1-\beta^{2}}\left(\left|\operatorname{grad}_{2 D} E_{z}\right|^{2}-j \frac{\omega \beta}{c_{0}}\left(\left(\mathbf{E}_{t}, \operatorname{grad}_{2 D} E_{z}\right)_{\mathbb{C}^{2}}-\left(\operatorname{grad}_{2 D} E_{z}, \mathbf{E}_{t}\right)_{\mathbb{C}^{2}}\right)+\frac{\omega^{2} \beta^{2}}{c_{0}^{2}}\left|\mathbf{E}_{t}\right|^{2}\right) \\
& -\int_{\Omega} \varepsilon_{r} \frac{1}{1-\beta^{2}}\left(\beta^{2}\left|\operatorname{grad}_{2 D} E_{z}\right|^{2}-j \frac{\omega \beta}{c_{0}}\left(\left(\mathbf{E}_{t}, \operatorname{grad}_{2 D} E_{z}\right)_{\mathbb{C}^{2}}-\left(\operatorname{grad}_{2 D} E_{z}, \mathbf{E}_{t}\right)_{\mathbb{C}^{2}}\right)+\frac{\omega^{2}}{c_{0}^{2}}\left|\mathbf{E}_{t}\right|^{2}\right),
\end{aligned}
$$

where ()$_{\mathbb{C}^{2}}$ denotes the usual scalar product of vectors in $\mathbb{C}^{2}$. Thus, the corresponding imaginary parts of interest in (4.8) are equal to

$$
\begin{gathered}
\int_{\Omega} \operatorname{Im}\left(\frac{1}{\mu_{r}}\right) \frac{1}{1-\beta^{2}}\left(\left|\operatorname{grad}_{2 D} E_{z}\right|^{2}+2 \frac{\omega \beta}{c_{0}} \operatorname{Im}\left(\left(\mathbf{E}_{t}, \operatorname{grad}_{2 D} E_{z}\right)_{\mathbb{C}^{2}}\right)+\frac{\omega^{2} \beta^{2}}{c_{0}^{2}}\left|\mathbf{E}_{t}\right|^{2}\right) \\
-\int_{\Omega} \operatorname{Im}\left(\varepsilon_{r}\right) \frac{1}{1-\beta^{2}}\left(\beta^{2}\left|\operatorname{grad}_{2 D} E_{z}\right|^{2}+2 \frac{\omega \beta}{c_{0}} \operatorname{Im}\left(\left(\mathbf{E}_{t}, \operatorname{grad}_{2 D} E_{z}\right)_{\mathbb{C}^{2}}\right)+\frac{\omega^{2}}{c_{0}^{2}}\left|\mathbf{E}_{t}\right|^{2}\right) .
\end{gathered}
$$

By using the Cauchy-Schwarz inequality we deduce $\left|\operatorname{Im}\left(\left(\mathbf{a}_{1}, \mathbf{a}_{2}\right)_{\mathbb{C}^{2}}\right)\right| \leq\left|\left(\mathbf{a}_{1}, \mathbf{a}_{2}\right)_{\mathbb{C}^{2}}\right| \leq\left|\mathbf{a}_{1}\right|\left|\mathbf{a}_{2}\right|$. Thus, for any real numbers $C_{1}, C_{2}$ and any $\mathbf{a}_{1}, \mathbf{a}_{2} \in \mathbb{C}^{2}$ we have $C_{1}^{2}\left|\mathbf{a}_{1}\right|^{2}+2 C_{1} C_{2} \operatorname{Im}\left(\left(\mathbf{a}_{1}, \mathbf{a}_{2}\right)_{\mathbb{C}^{2}}\right)+C_{2}^{2}\left|\mathbf{a}_{2}\right|^{2} \geq C_{1}^{2}\left|\mathbf{a}_{1}\right|^{2}-$ $2\left|C_{1}\right|\left|C_{2}\right|\left|\operatorname{Im}\left(\left(\mathbf{a}_{1}, \mathbf{a}_{2}\right)_{\mathbb{C}^{2}}\right)\right|+C_{2}^{2}\left|\mathbf{a}_{2}\right|^{2} \geq C_{1}^{2}\left|\mathbf{a}_{1}\right|^{2}-2\left|C_{1}\right|\left|C_{2}\right|\left|\mathbf{a}_{1}\right|\left|\mathbf{a}_{2}\right|+C_{2}^{2}\left|\mathbf{a}_{2}\right|^{2}=\left(C_{1}\left|\mathbf{a}_{1}\right|-C_{2}\left|\mathbf{a}_{2}\right|\right)^{2} \geq 0$. Thus, by identifying in one case $C_{1}$ with 1 and $C_{2}$ with $\frac{\omega \beta}{c_{0}}$ and, in the other case, $C_{1}$ with $\beta$ and $C_{2}$ with $\frac{\omega}{c_{0}}$ we conclude that the quantities contained in the large brackets in the two integrals in (4.10) are not negative. Moreover, taking account of HV4, the considered integrals provide non-negative results whenever HM6 holds 
true. The same integrals give strictly positive contributions whenever HM6 and HM7 holds true, if $\mathbf{E}_{t} \neq 0$ or $\operatorname{grad}_{2 D} E_{z} \neq 0$ in $D$.

We have verified that all terms appearing in (4.8) are not negative if HM6 and HB2 hold true. Moreover, some of them could be strictly positive if, in addition to HB2, HB3 holds true, provided that $\gamma_{0} E_{z} \neq 0$ or $\mathbf{E}_{t} \cdot \mathbf{l} \neq 0$ on $\Gamma_{l}$. Finally, strictly positive contributions are obtained if, in addition to HM6, the part relative to $\varepsilon_{r}$ of HM7 holds true, provided that $E_{z} \neq 0$ or $\operatorname{grad}_{2 D} E_{z} \neq 0$ or $\mathbf{E}_{t} \neq 0$ in $D$. Analogously, if the part relative to $\mu_{r}$ of HM7 holds true, the same result is obtained provided that $\operatorname{grad}_{2 D} E_{z} \neq 0$ or $\mathbf{E}_{t} \neq 0$ or $\operatorname{curl}_{2 D} \mathbf{E}_{t} \neq 0$ in $D$.

By using Lemma 4.4 one easily gets a stronger result for regions containing motionless media.

Lemma 4.5. Under the additional hypotheses HM6 and HB2, any solution $\mathbf{E}$ of Problem 2 with $l=0$ satisfies $\mathbf{E}=0$ in $\Omega_{k}, k \in M_{0}$, if HB3 or HM7 and HM8 hold true whenever $\Omega_{k}$ contains regions with lossy media or the media in $\Omega_{k}$ are lossless but $\Omega_{k}$ has a non-empty, open, Lipschitz continuous part of its boundary in common with $\Gamma_{l}$ or with the boundary of a subregion $\Omega_{j}$ where $\mathbf{E}=0$ has already been proved.

Proof. First of all, we show that $\mathbf{E}=0$ in $D$ even if just the part relative to $\mu_{r}$ of HM7 holds true. As a matter of fact, let us consider $w_{z}$ as a very regular function having support in $D$ and $\mathbf{w}_{t}=0$. Then, from (3.11), taking account that $\operatorname{grad}_{2 D} E_{z}=0, \mathbf{E}_{t}=0$ and $\operatorname{curl}_{2 D} \mathbf{E}_{t}=0$ in $D$, we deduce $0=a(\mathbf{E}, \mathbf{w})=-\frac{\omega^{2}}{c_{0}^{2}}\left(\varepsilon_{r} E_{z}, w_{z}\right)_{0, D}$ which gives the desired result for the density of the space of the indicated test functions in $L^{2}(D)$, under the indicated hypotheses.

Thus, overall, under hypotheses HM6 and HB2, the solution $\mathbf{E}$ of the homogeneous problem satisfies $\mathbf{E}=0$ in $D$ if HM7 and HM8 hold true and $\gamma_{0} E_{z}=0$ and $\mathbf{E}_{t} \cdot \mathbf{l}=0$ on $\Gamma_{l}$ if HB3 holds true.

Now we proceed as in ([18], p. 92 and [22]) and apply unique continuation results. Our assumptions related to the domain (HD1), its subdomains (HD3), their boundaries (HD2, HD3) and the material properties of motionless media (HM1-HM3 and HM5-HM8) are taken from [22] and are slightly stronger that those considered in ([18], p. 83 and p. 94) as far as the regularity of $\varepsilon_{r}$ is concerned (HM3).

For this reason we can proceed exactly as in $[18,22]$. In this way we can firstly show that $\mathbf{E}=0$ in any subdomain $\Omega_{k}, k \in M_{0}$, which contains regions with lossy motionless media, as considered in hypothesis HM7. Then it is possible to prove that $\mathbf{E}=0$ in any subdomain $\Omega_{k}, k \in M_{0}$, which contains lossless and motionless media and having a non-empty, open Lipschitz continuous part of its boundary in common with $\Gamma_{l}$ if HM6, HB2 and HB3 hold true or with the boundary of a subregion $\Omega_{j}$ where $\mathbf{E}=0$ has already been proved.

Unfortunately, these considerations do not allow to obtain the uniqueness result of interest since we still have to deal with subdomains $\Omega_{j}$ where passive and lossless moving media are present. The reader should remember that we do not consider the trivial case of scatterers moving with a uniform axial speed in vacuum, which can be managed with all matter at rest, as pointed out in the Introduction.

The next lemma gives us the last intermediate result we need to prove Theorem 4.3.

Lemma 4.6. Under the additional hypotheses HB2, HM6, HM8, HMV1 and HMV2 any solution $\mathbf{E}$ of Problem 2 with $l=0$ satisfies $\mathbf{E}=0$ in $\Omega_{i}, i \in M_{\beta}$ whenever $\Omega_{i}$ is adjacent to a region $\Omega_{k}, k \in M$, where it has already been proved that $\mathbf{E}=0$, or $\partial \Omega_{i}$ shares a non-empty, open, Lipschitz continuous part with $\Gamma_{l}$ and HB3 holds true.

Proof. In order to deal with the subdomains involving moving media we study under which conditions it is possible to apply the results proved in [17] and, then, deduce the analyticity of the fields. To the best of authors' knowledge, this is the only way to prove a unique continuation results in the presence of moving (and, then, bianisotropic) media.

With this aim, let us consider the subdomain $\Omega_{i}, i \in M_{\beta}$, containing moving media and suppose that $\mathbf{E}$ is a solution of the homogeneous variational problem, as usual. From it we deduce, $\mathbf{B}, \mathbf{H}$ and $\mathbf{D}$ by using (3.1)(3.5), with $\mathbf{J}_{m}=0$, and (2.2). We consider the three-dimensional domain $\Omega_{i 3 D}=\Omega_{i} \times(0,1), i \in M_{\beta}$, and 
the three-dimensional fields, defined in the three-dimensional domain $\Omega_{i 3 D}$ but independent of the $z$-coordinate $\mathbf{E}_{3 D}=\mathbf{E}, \mathbf{B}_{3 D}=\mathbf{B}, \mathbf{H}_{3 D}=\mathbf{H}$ and $\mathbf{D}_{3 D}=\mathbf{D}$ in $\Omega_{i 3 D}$. Since $\mathbf{E} \in U, \mathbf{B} \in\left(L^{2}(\Omega)\right)^{3}, \mathbf{H} \in U$ and $\mathbf{D} \in\left(L^{2}(\Omega)\right)^{3}$, we easily get $\mathbf{E}_{3 D} \in H\left(\operatorname{curl}, \Omega_{i 3 D}\right), \mathbf{B}_{3 D} \in\left(L^{2}\left(\Omega_{i 3 D}\right)\right)^{3}, \mathbf{H}_{3 D} \in H\left(\operatorname{curl}, \Omega_{i 3 D}\right), \mathbf{D}_{3 D} \in\left(L^{2}\left(\Omega_{i 3 D}\right)\right)^{3}$. Moreover, by using Theorem 3.2 it is almost immediate to deduce that the homogeneous equations $(2.13)_{1},(2.13)_{2},(2.13)_{3}$ and $(2.13)_{4}$ imply the validity of the three-dimensional homogeneous Maxwell equations

$$
\begin{cases}\nabla \times \mathbf{H}_{3 D}-j \omega \mathbf{D}_{3 D}=0 & \text { in } \Omega_{i 3 D} \\ \nabla \times \mathbf{E}_{3 D}+j \omega \mathbf{B}_{3 D}=0 & \text { in } \Omega_{i 3 D} .\end{cases}
$$

Then, since $\omega>0, \mathbf{B}_{3 D} \in H\left(\operatorname{div}^{0}, \Omega_{i 3 D}\right)$ and $\mathbf{D}_{3 D} \in H\left(\operatorname{div}^{0}, \Omega_{i 3 D}\right)$. Thus, $\left(\mathbf{E}_{3 D}, \mathbf{B}_{3 D}, \mathbf{H}_{3 D}, \mathbf{D}_{3 D}\right) \in$ $H\left(\operatorname{curl}, \Omega_{i 3 D}\right) \times H\left(\operatorname{div}^{0}, \Omega_{i 3 D}\right) \times H\left(\operatorname{curl}, \Omega_{i 3 D}\right) \times H\left(\operatorname{div}^{0}, \Omega_{i 3 D}\right)$ as it was assumed in [17] (we identify $\Omega_{i 3 D}$ with $\Omega$ of [17]). $\mathbf{J}_{e}$ and $\mathbf{J}_{m}$ are equal to zero in the homogeneous problem of interest in this section so that the corresponding quantities in (4.11), which were considered in [17], are trivial and we easily get that they have the regularity required by HS1 of [17], that is $\mathbf{J}_{e}, \mathbf{J}_{m} \in H\left(\operatorname{div}^{0}, \Omega_{i 3 D}\right)$, or by any other statement of that paper.

Moreover, from (2.1)-(2.4) one easily gets

$$
\begin{cases}\mathbf{D}_{3 D}=\left(1 / c_{0}\right) P \mathbf{E}_{3 D}+L \mathbf{B}_{3 D} & \text { in } \Omega_{i 3 D} \\ \mathbf{H}_{3 D}=M \mathbf{E}_{3 D}+c_{0} Q \mathbf{B}_{3 D} & \text { in } \Omega_{i 3 D},\end{cases}
$$

where

$$
\begin{aligned}
& P=\sqrt{\frac{\varepsilon_{0}}{\mu_{0}}}\left[\begin{array}{ccc}
\frac{1+\mu_{r} \varepsilon_{r}-\zeta_{1}}{\mu_{r}} & 0 & 0 \\
0 & \frac{1+\mu_{r} \varepsilon_{r}-\zeta_{1}}{\mu_{r}} & 0 \\
0 & 0 & \varepsilon_{r}
\end{array}\right], \\
& Q=\sqrt{\frac{\varepsilon_{0}}{\mu_{0}}}\left[\begin{array}{ccc}
\frac{\zeta_{1}}{\mu_{r}} & 0 & 0 \\
0 & \frac{\zeta_{1}}{\mu_{r}} & 0 \\
0 & 0 & \frac{1}{\mu_{r}}
\end{array}\right], \\
& L=M=\sqrt{\frac{\varepsilon_{0}}{\mu_{0}}}\left[\begin{array}{ccc}
0 & -\frac{\zeta_{2}}{\mu_{r}} & 0 \\
\frac{\zeta_{2}}{\mu_{r}} & 0 & 0 \\
0 & 0 & 0
\end{array}\right] .
\end{aligned}
$$

In equations (4.13)-(4.15) we have not changed the symbols for the fields $\varepsilon_{r}, \mu_{r}, \beta, \zeta_{1}$ and $\zeta_{2}$, but the reader should consider that, in the indicated equations, these fields are defined on $\Omega_{i 3 D}$ and are assumed to be independent of the $z$ coordinate. In the following the reader should refer to the quantities defined on the threedimensional domain when they are involved in considerations aiming at the applications of the results of [17].

By using (5.5) of [17], after some trivial calculation we obtain

$$
\begin{aligned}
& \kappa=\frac{1}{\varepsilon_{0}}\left[\begin{array}{ccc}
\frac{\mu_{r}}{1+\mu_{r} \varepsilon_{r}-\zeta_{1}} & 0 & 0 \\
0 & \frac{\mu_{r}}{1+\mu_{r} \varepsilon_{r}-\zeta_{1}} & 0 \\
0 & 0 & \frac{1}{\varepsilon_{r}}
\end{array}\right], \\
& \nu=\frac{1}{\mu_{0}}\left[\begin{array}{ccc}
\frac{\zeta_{1}}{\mu_{r}}+\frac{\zeta_{2}^{2}}{\mu_{r}\left(1+\mu_{r} \varepsilon_{r}-\zeta_{1}\right)} & 0 & 0 \\
0 & \frac{\zeta_{1}}{\mu_{r}}+\frac{\zeta_{2}^{2}}{\mu_{r}\left(1+\mu_{r} \varepsilon_{r}-\zeta_{1}\right)} & 0 \\
0 & 0 & \frac{1}{\mu_{r}}
\end{array}\right], \\
& \gamma=-\chi=c_{0}\left[\begin{array}{ccc}
0 & -\frac{\zeta_{2}}{1+\mu_{r} \varepsilon_{r}-\zeta_{1}} & 0 \\
-\frac{\zeta_{2}}{1+\mu_{r} \varepsilon_{r}-\zeta_{1}} & 0 & 0 \\
0 & 0 & 0
\end{array}\right] .
\end{aligned}
$$


In [17] it has been assumed that $\kappa, \chi, \gamma$ and $\nu$ satisfy HM1 of [17], that is they have continuous and bounded entries in the subdomain where the regularity of the field is of interest. We know that $\mu_{r}, \frac{1}{\mu_{r}}, \frac{1}{\varepsilon_{r}}$ are very regular and bounded by HM3, HM5 and HM8. $\zeta_{1}$ and $\zeta_{2}$ are very regular and bounded, too, by HV2, HM3 and HV4. Thus we achieve the result of interest if $\left(1+\mu_{r} \varepsilon_{r}-\zeta_{1}\right)^{-1}$ is bounded. By using (2.5) we have $1+\mu_{r} \varepsilon_{r}-\zeta_{1}=1+\mu_{r} \varepsilon_{r}-\frac{1-\mu_{r} \varepsilon_{r} \beta^{2}}{1-\beta^{2}}=\frac{\mu_{r} \varepsilon_{r}-\beta^{2}}{1-\beta^{2}}$. Thus, the required boundedness is guaranteed whenever HMV1 holds true.

From (4.16) and (4.17) it is easy to check that

$$
\begin{gathered}
\text { determinant }(\kappa)=\frac{\mu_{r}^{2}}{\varepsilon_{0}^{3} \varepsilon_{r}\left(1+\mu_{r} \varepsilon_{r}-\zeta_{1}\right)^{2}}=\frac{\mu_{r}^{2}\left(1-\beta^{2}\right)^{2}}{\varepsilon_{0}^{3} \varepsilon_{r}\left(\mu_{r} \varepsilon_{r}-\beta^{2}\right)^{2}} \\
\text { determinant }(\nu)=\frac{1}{\mu_{0}^{3} \mu_{r}^{3}}\left(\zeta_{1}+\frac{\zeta_{2}^{2}}{1+\mu_{r} \varepsilon_{r}-\zeta_{1}}\right)^{2}=\frac{\varepsilon_{r}^{2}\left(1-\beta^{2}\right)^{2}}{\mu_{0}^{3} \mu_{r}\left(\mu_{r} \varepsilon_{r}-\beta^{2}\right)^{2}} .
\end{gathered}
$$

The entries of $\kappa$ and $\nu$ together with the corresponding determinants, determinant $(\kappa)$ and determinant $(\nu)$, can be extended by continuity to $\bar{\Omega}_{i 3 D}$, for all $i \in M$, under hypotheses HV2, HM3, HM5, HM8, HMV1. Thus we can define the constant quantities $C_{\kappa, d}$ and $C_{\nu, d}$ of [17] as $C_{\kappa, d, i}=\min _{\mathbf{x} \in \bar{\Omega}_{i 30}}(|\operatorname{determinant}(\kappa)|), C_{\nu, d, i}=$ $\min _{\mathbf{x} \in \bar{\Omega}_{i 3 D}}(|\operatorname{determinant}(\nu)|), i \in M_{\beta}$. By using (4.19) and (4.20) we easily get (4.4) and (4.5), respectively. Then HM8 of [17], which requires the existence of $C_{\kappa, d}$ and $C_{\nu, d}$, both larger than zero, such that $C_{\kappa, d} \leq$ $\mid$ determinant $(\kappa)\left|, C_{\nu, d} \leq\right|$ determinant $(\nu) \mid$ everywhere in the domain of interest, holds true in $\Omega_{i 3 D}$ for all $i \in M_{\beta}$ under hypotheses HV2, HM3, HM5, HM8, HMV1 and HV4 (see also Rem. 4.2).

Exploiting again the diagonal structure of $\kappa$ and $\nu$ and the continuity on $\bar{\Omega}_{i 3 D}$ of their entries, for all $i \in M$, we can define the constant quantities $C_{\kappa, s}$ and $C_{\nu, s}$ of [17] as

$$
\begin{gathered}
C_{\kappa, s, i}=\frac{1}{\varepsilon_{0}} \max _{\mathbf{x} \in \bar{\Omega}_{i 3 D}}\left(\max \left(2\left|\frac{\mu_{r}}{1+\mu_{r} \varepsilon_{r}-\zeta_{1}}\right|,\left|\frac{\mu_{r}}{1+\mu_{r} \varepsilon_{r}-\zeta_{1}}\right|+\frac{1}{\left|\varepsilon_{r}\right|}\right)\right), \\
C_{\nu, s, i}=\frac{1}{\mu_{0}} \max _{\mathbf{x} \in \bar{\Omega}_{i 3 D}}\left(\max \left(2\left|\frac{\zeta_{1}}{\mu_{r}}+\frac{\zeta_{2}^{2}}{\mu_{r}\left(1+\mu_{r} \varepsilon_{r}-\zeta_{1}\right)}\right|,\left|\frac{\zeta_{1}}{\mu_{r}}+\frac{\zeta_{2}^{2}}{\mu_{r}\left(1+\mu_{r} \varepsilon_{r}-\zeta_{1}\right)}\right|+\frac{1}{\left|\mu_{r}\right|}\right)\right),
\end{gathered}
$$

$i \in M_{\beta}$, and, by using (2.5) and (2.6), we get expressions (4.2) and (4.3), respectively.

Again from (4.16) and (4.17) we easily deduce that $\kappa^{-1}$ and $\nu^{-1}$ are diagonal matrices with continuous entries which can be extended by continuity to $\bar{\Omega}_{i 3 D}$, for all $i \in M$, under hypotheses HV2, HM3, HM5 and HV4. For this reason we can define the constant quantities $C_{\kappa, r}$ and $C_{\nu, r}$ of [17] as

$$
\begin{gathered}
C_{\kappa, r, i}=\varepsilon_{0} \min \left(\min _{\mathbf{x} \in \bar{\Omega}_{i 3 D}}\left|\frac{1+\mu_{r} \varepsilon_{r}-\zeta_{1}}{\mu_{r}}\right|, \min _{\mathbf{x} \in \bar{\Omega}_{i 3 D}}\left|\varepsilon_{r}\right|\right), \\
C_{\nu, r, i}=\mu_{0} \min \left(\min _{\mathbf{x} \in \bar{\Omega}_{i 3 D}}\left|\frac{\mu_{r}}{\zeta_{1}+\frac{\zeta_{2}^{2}}{1+\mu_{r} \varepsilon_{r}-\zeta_{1}}}\right|, \min _{\mathbf{x} \in \bar{\Omega}_{i 3 D}}\left|\mu_{r}\right|\right),
\end{gathered}
$$

$i \in M_{\beta}$. Once more, by using (2.5) and (2.6), we get expressions (4.6) and (4.7), respectively. Then HM9 of [17], that is $\exists \exists C_{\kappa, r}>0, C_{\nu, r}>0:\left|\mathbf{l}_{1,3, n}^{T} \kappa^{-1} \mathbf{l}_{1,3, n}\right| \geq C_{\kappa, r},\left|\mathbf{l}_{1,3, n}^{T} \nu^{-1} \mathbf{l}_{1,3, n}\right| \geq C_{\nu, r}$ everywhere in the domain of interest, $\forall \mathbf{l}_{1,3, n} \in \mathbb{R}^{3}$ having a unit Euclidean norm, holds true in $\Omega_{i 3 D}$ for all $i \in M_{\beta}$ under assumptions HV2, HM3, HM5, HM8, HMV1 and HV4 (see also Rem. 4.2).

Having already verified that HM1, HS1, HM8 and HM9 of [17] hold true in $\Omega_{i 3 D}$ for all $i \in M_{\beta}$, in order to be able to apply Theorem 7.3 of [17], we have to consider condition (7.11) of the same paper. We report here this condition to ease the reader task

$$
\frac{4\left(\left(\sum_{i, j=1}^{3}\left|\gamma_{i j}\right|\right)-\min _{i=1,2,3}\left|\gamma_{i i}\right|\right)\left(\left(\sum_{i, j=1}^{3}\left|\chi_{i j}\right|\right)-\min _{i=1,2,3}\left|\chi_{i i}\right|\right)}{\left(-C_{\kappa, s, i}+\sqrt{C_{\kappa, s, i}^{2}+4 C_{\kappa, d, i} C_{\kappa, r, i}}\right)\left(-C_{\nu, s, i}+\sqrt{C_{\nu, s, i}^{2}+4 C_{\nu, d, i} C_{\nu, r, i}}\right)}<1 \text { in } \Omega_{i 3 D}, \forall i \in M_{\beta} .
$$


In the case of interest, by using (4.18) we deduce $\gamma_{i i}=\chi_{i i}=0, i=1,2,3$, and

$$
\sum_{i, j=1}^{3}\left|\gamma_{i j}\right|=\sum_{i, j=1}^{3}\left|\chi_{i j}\right|=2 c_{0}\left|\frac{\zeta_{2}}{1+\mu_{r} \varepsilon_{r}-\zeta_{1}}\right|=2 c_{0}\left|\frac{\beta\left(\mu_{r} \varepsilon_{r}-1\right)}{\mu_{r} \varepsilon_{r}-\beta^{2}}\right| .
$$

Taking account of (4.26) condition (4.25) (that is condition (7.11) of [17]) becomes condition (4.1).

Now we can fully exploit the results of [17] and prove that $\left(\mathbf{E}_{3 D}, \mathbf{B}_{3 D}, \mathbf{H}_{3 D}, \mathbf{D}_{3 D}\right)$ is done of fields equal to zero in $\Omega_{i 3 D}, i \in M_{\beta}$, if

- $\Omega_{i}$ is adjacent to a region $\Omega_{k}, k \in M$, where it has already been proved that $\mathbf{E}=0$; or

- $\partial \Omega_{i}$ shares a non-empty, open, Lipschitz continuous part with $\Gamma_{l}$.

As a matter of fact, we proceed as in ([18], p. 94). In both cases we introduce a sufficiently small open ball $B \subset \mathbb{R}^{2}$ centered on a point of $\bar{\Omega}_{i} \cap \bar{\Omega}_{k}$ or on a point of $\partial \Omega_{i} \cap \Gamma_{l}$.

First of all, we remember that $\mathbf{E}$ is a solution of the homogeneous version of Problem 2 (i.e., the one with $l=0$ in Eq. (3.13)). From this solution, by using (3.1)-(3.5) and (2.2), we deduce $\mathbf{B}, \mathbf{H}$ and $\mathbf{D}$. By Theorem 3.2 $(\mathbf{E}, \mathbf{B}, \mathbf{H}, \mathbf{D})$ is a solution of the homogeneous version of Problem 1 (i.e., the one $\mathbf{J}_{e}=0, \mathbf{J}_{m}=0, f_{R l}=0$, $f_{R z}=0$ in Eq. (2.13)). Since in both cases we have to deal with we have $\gamma_{0} E_{z}=0$ and $\mathbf{E}_{t} \cdot \mathbf{l}=0$ on $B \cap \partial \Omega_{i}$, by $(2.13)_{5}$ and $(2.13)_{6}$ (with $f_{R l}=0$ and $f_{R z}=0$ ), we deduce $\gamma_{0} H_{z}=0$ and $\mathbf{H}_{t} \cdot \mathbf{l}=0$ on $B \cap \partial \Omega_{i}$.

Moreover, taking account of $(2.13)_{2}$ and $(2.13)_{4}$ with $\mathbf{J}_{e t}=0$ and $\mathbf{J}_{m t}=0$, the conditions $\gamma_{0} E_{z}=0$ and $\gamma_{0} H_{z}=0$ on $B \cap \partial \Omega_{i}$ imply $\gamma_{n} \mathbf{D}_{t}=0$ and $\gamma_{n} \mathbf{B}_{t}=0$ on $B \cap \partial \Omega_{i}$, where $\gamma_{n} \mathbf{A}_{t}$ denotes the boundary values of the normal component to the boundary of a sufficiently regular $\mathbf{A}_{t}$ ([32], p. 204). The indicated boundary conditions have a meaning because $\hat{\mathbf{z}} \times \mathbf{D}_{t}=\frac{1}{j \omega} \operatorname{grad}_{2 D} H_{z}$ and $\hat{\mathbf{z}} \times \mathbf{B}_{t}=-\frac{1}{j \omega} \operatorname{grad}_{2 D} E_{z}$ are in $\left(L^{2}(\Omega)\right)^{2}$ and have a well defined $\operatorname{curl}_{2 D}$ (equal to 0 in $\Omega$ ).

Finally, let us define $B_{3 D}=B \times(0,1)$ and extend in an analytical way from $\Omega_{i 3 D}$ to $B_{3 D} \backslash \bar{\Omega}_{i 3 D}$ the fields $\varepsilon_{r}$, $\mu_{r}$ and $\beta$ (this is possible by HV2 and HM3 and by taking account of HM4). In both cases we have to consider $\mathbf{E}_{3 D}, \mathbf{B}_{3 D}, \mathbf{H}_{3 D}$, and $\mathbf{D}_{3 D}$ are either trivial fields in $B_{3 D} \backslash \bar{\Omega}_{i 3 D}$ (in the first of the two cases) or can be extended to $B_{3 D} \backslash \bar{\Omega}_{i 3 D}$ as trivial fields (in the second of the two cases). Then, by Theorem 7.3 of [17] we obtain $\mathbf{E}_{3 D}=0$, $\mathbf{B}_{3 D}=0, \mathbf{H}_{3 D}=0$, and $\mathbf{D}_{3 D}=0$ in $B_{3 D}$ since these fields are analytic in $B_{3 D}$ and they are equal to zero in $B_{3 D} \backslash \bar{\Omega}_{i 3 D}$. We can deduce the analyticity of the fields in $B_{3 D}$ if HMV1 and HMV2 are satisfied because:

- the fields $\mathbf{E}_{3 D}, \mathbf{B}_{3 D}, \mathbf{H}_{3 D}, \mathbf{D}_{3 D}$ satisfy (4.11) in $B_{3 D}$ since (4.11) holds true in $\Omega_{i 3 D}$ and in $B_{3 D} \backslash \bar{\Omega}_{i 3 D}$;

- the fields $\mathbf{E}_{3 D}, \mathbf{B}_{3 D}, \mathbf{H}_{3 D}, \mathbf{D}_{3 D}$ satisfy (4.12) in $B_{3 D}$ since (4.12) holds true in $\Omega_{i 3 D}$ and in $B_{3 D} \backslash \bar{\Omega}_{i 3 D}$;

- by using the properties of the boundary values deduced above we easily conclude that in both cases of interest $\left(\mathbf{E}_{3 D}, \mathbf{B}_{3 D}, \mathbf{H}_{3 D}, \mathbf{D}_{3 D}\right) \in H\left(\operatorname{curl}, B_{3 D}\right) \times H\left(\operatorname{div}^{0}, B_{3 D}\right) \times H\left(\operatorname{curl}, B_{3 D}\right) \times H\left(\operatorname{div}^{0}, B_{3 D}\right)$;

- HS1 of [17] is satisfied in any case;

- HM1, HM8 and HM9 of [17] are satisfied by the fields $\varepsilon_{r}, \mu_{r}$ and $\beta$ extended as indicated above in any sufficiently small ball $B_{3 D}$ since we have verified them in $\bar{\Omega}_{i 3 D}$ and all the extended quantities involved are at least $C^{2}\left(\bar{\Omega}_{i 3 D}\right)$;

- HMV2 implies that (7.11) of [17] is satisfied by the fields $\varepsilon_{r}, \mu_{r}$ and $\beta$ extended as indicated above in any sufficiently small ball $B_{3 D}$ since we have verified it in $\bar{\Omega}_{i 3 D}$ and all the extended quantities involved are at least $C^{2}\left(\bar{\Omega}_{i 3 D}\right)$.

Once the fields $\mathbf{E}_{3 D}, \mathbf{B}_{3 D}, \mathbf{H}_{3 D}, \mathbf{D}_{3 D}$ are proved to be equal to zero in $B_{3 D}$ we easily get $\mathbf{E}_{3 D}=0, \mathbf{B}_{3 D}=0$, $\mathbf{H}_{3 D}=0$, and $\mathbf{D}_{3 D}=0$ in $\Omega_{i 3 D}$ by the analyticity of the indicated fields in $\Omega_{i 3 D}$. This property is easily deduced under hypotheses HMV1 and HMV2 by a direct and easier application of Theorem 7.3 of [17] since in $\Omega_{i 3 D}$ all its hypotheses have been directly verified. Then $\mathbf{E}=0, \mathbf{B}=0, \mathbf{H}=0$, and $\mathbf{D}=0$ in $\Omega_{i}$.

Thus we have shown that we are able to manage regions involving motionless or moving media. Therefore, the result of interest, that is $\mathbf{E}=0$ in $\Omega$, can be deduced by considering all subdomains, as shown in ([18], p. 95). This completes the proof of Theorem 4.3. 


\subsection{Uniqueness of the solution for the problem defined by the adjoint sesquilinear form $a^{*}$}

The adjoint sesquilinear form $a^{*}$ is defined by ([38], p. 353)

$$
a^{*}(\mathbf{w}, \mathbf{u})=(a(\mathbf{u}, \mathbf{w}))^{*} \quad \forall \mathbf{u}, \mathbf{w} \in U
$$

where $(p)^{*}$ denotes the complex conjugate of $p \in \mathbb{C}$. In the particular case of interest we have

$$
\begin{aligned}
a^{*}(\mathbf{w}, \mathbf{u})= & \left(\left(\frac{\zeta_{1}}{\mu_{r}}\right)^{*} \operatorname{grad}_{2 D} w_{z}, \operatorname{grad}_{2 D} u_{z}\right)_{0, \Omega}+\left(\left(\frac{1}{\mu_{r}}\right)^{*} \operatorname{curl}_{2 D} \mathbf{w}_{t}, \operatorname{curl}_{2 D} \mathbf{u}_{t}\right)_{0, \Omega} \\
& -j \frac{\omega}{c_{0}}\left(\left(\frac{\zeta_{2}}{\mu_{r}}\right)^{*} \mathbf{w}_{t}, \operatorname{grad}_{2 D} u_{z}\right)_{0, \Omega}+j \frac{\omega}{c_{0}}\left(\left(\frac{\zeta_{2}}{\mu_{r}}\right)^{*} \operatorname{grad}_{2 D} w_{z}, \mathbf{u}_{t}\right)_{0, \Omega} \\
& -\frac{\omega^{2}}{c_{0}^{2}}\left(\left(\varepsilon_{r}\right)^{*} w_{z}, u_{z}\right)_{0, \Omega}-\frac{\omega^{2}}{c_{0}^{2}}\left(\left(\frac{1+\varepsilon_{r} \mu_{r}-\zeta_{1}}{\mu_{r}}\right)^{*} \mathbf{w}_{t}, \mathbf{u}_{t}\right)_{0, \Omega} \\
& -j \omega \mu_{0}\left((Y)^{*}\left(\gamma_{0} w_{z}\right), \gamma_{0} u_{z}\right)_{0, \Gamma}-j \omega \mu_{0}\left((Y)^{*}\left(\mathbf{w}_{t} \cdot \mathbf{l}\right), \mathbf{u}_{t} \cdot \mathbf{l}\right)_{0, \Gamma} .
\end{aligned}
$$

With this definition one can refer to the following adjoint homogeneous problem.

Problem 3. Under the hypotheses HD1-HD3, HV1-HV4, HM1-HM4, HB1, given $\omega>0$, find $\mathbf{w} \in U$ such that

$$
a^{*}(\mathbf{w}, \mathbf{u})=0 \quad \forall \mathbf{u} \in U .
$$

Like in [22], it is now extremely simple to deduce

Theorem 4.7. Under the hypotheses HD1-HD3, HV1-HV4, HM1-HM6, HM8, HB1-HB2, HMV1 and HMV2, Problem 3 admits a unique solution $\mathbf{w}=0$ provided that at least one of HM7 and HB3 is satisfied.

Proof. If HB3 is satisfied we deduce $\gamma_{0} E_{z}=0$ and $\mathbf{E}_{t} \cdot \mathbf{l}=0$ on $\Gamma_{l}$ because all imaginary parts of $a^{*}$ retain the same signs as before (even though all these signs are opposite with respect to those of $a$ ). In the subdomains involving motionless and lossy media, where HM7 holds true, we can proceed exactly as in [22] and deduce $\mathbf{w}=0$ there (essentially, for the common sign of the imaginary parts of $a^{*}$ as before). In the other regions, including those where the media are in motion, all constitutive parameters are real-valued fields and all deductions obtained in Section 4.1 apply in this case, too.

With this result we immediately deduce that

Theorem 4.8. Under the hypotheses HD1-HD3, HV1-HV4, HM1-HM6, HM8, HB1-HB2, HMV1 and HMV2, the following condition holds true

$$
\text { for every } \mathbf{w} \in U, \mathbf{w} \neq 0, \quad \sup _{\mathbf{u} \in U}|a(\mathbf{u}, \mathbf{w})|>0
$$

provided that at least one of HM7 and HB3 is satisfied.

Proof. Suppose that (4.30) is not satisfied. Then we can find $\mathbf{w} \in U, \mathbf{w} \neq 0$ such that $\sup _{\mathbf{u} \in U}|a(\mathbf{u}, \mathbf{w})|=0$. But by using (4.27) $|a(\mathbf{u}, \mathbf{w})|=\left|(a(\mathbf{u}, \mathbf{w}))^{*}\right|=\left|a^{*}(\mathbf{w}, \mathbf{u})\right|$. Then, if (4.30) does not hold true, we can find $\mathbf{w} \in U, \mathbf{w} \neq 0$ such that $\sup _{\mathbf{u} \in U}\left|a^{*}(\mathbf{w}, \mathbf{u})\right|=0$. Thus, for the indicated $\mathbf{w} \neq 0,\left|a^{*}(\mathbf{w}, \mathbf{u})\right|=0 \forall \mathbf{u} \in U$. This is at odds with Theorem 4.7, since we have assumed the same hypotheses. 


\subsection{Additional hypotheses for proving the inf-sup condition}

The first of the new hypotheses we will consider in this subsection is:

HMV3. the fields $\mu_{r}, \varepsilon_{r}$ and $\beta$ allow to find $C_{m v 3}>0$ such that $\frac{\mu_{r} \varepsilon_{r}-\beta^{2}}{\mu_{r}\left(1-\beta^{2}\right)} \geq C_{m v 3}$ in $\Omega_{k}, \forall k \in M_{\beta}$, and $\operatorname{Re}\left(\varepsilon_{r}\right) \geq C_{m v 3}$ in $\Omega_{k}, \forall k \in M_{0}$.

Remark 4.9. Where the media are at rest we have $\frac{1+\varepsilon_{r} \mu_{r}-\zeta_{1}}{\mu_{r}}=\frac{\mu_{r} \varepsilon_{r}-\beta^{2}}{\mu_{r}\left(1-\beta^{2}\right)}=\varepsilon_{r}$. In $\Omega_{k}, \forall k \in M_{\beta}, \frac{\mu_{r} \varepsilon_{r}-\beta^{2}}{\mu_{r}\left(1-\beta^{2}\right)}$ is real valued by HM3 and HV2.

Remark 4.10. HMV3 is a new independent hypothesis. For example, it prevents the presence of traditional media and the so-called epsilon-negative or double-negative materials [37] among those which are at rest in the selected reference frame. All other hypotheses encountered so far do not exclude such a combination of materials.

Remark 4.11. Hypothesis HMV3 could also be written to include cases in which the fields $\mu_{r}, \varepsilon_{r}$ and $\beta$ are such that $\frac{\mu_{r} \varepsilon_{r}-\beta^{2}}{\mu_{r}\left(1-\beta^{2}\right)} \leq-C_{m v 3}$ in $\Omega_{k}, \forall k \in M_{\beta}$, and $\operatorname{Re}\left(\varepsilon_{r}\right) \leq-C_{m v 3}$ in $\Omega_{k}, \forall k \in M_{0}$. We do not consider these cases because, on the one hand, the generality of our results is not reduced in a significant way and, on the other hand, the mathematical developments can be limited to a reasonable extent. An analogous consideration applies, for example, to the following condition HM9.

The following two additional hypotheses refer, respectively, to the relative permeability and to the admittance involved in boundary conditions:

HM9. the field $\mu_{r}$ is such that $\operatorname{Re}\left(\frac{1}{\mu_{r}}\right) \geq C_{\mu M k}>0$ in $\Omega_{k}, k \in M . C_{\mu M}=\min _{k \in M} C_{\mu M k}$.

This hypothesis prevents the involvement of the so-called mu-negative or double-negative materials [37] in the class of problems we deal with. Together with HMV3 (see also Rem. 4.10) it prevents the possibility of dealing with all types of isotropic metamaterials [37]. However, the reader should notice that the main difficulties we had to overcome in this paper are not due to the type of materials but to their axial movements (which make these media appear as bianisotropic materials in the chosen reference frame). Moreover, it is now very wellknown [39-44], that the presence of traditional media and metamaterials can give rise to ill-posed problems even when all media are at rest in the selected reference frame.

HB3S. we can find $C_{Y m}>0$ such that $\operatorname{Re}(Y) \geq C_{Y m}$ almost everywhere on $\Gamma$.

This condition is a strengthened version of hypothesis HB3, which does not introduce any significant restriction for practical applications, since it allows the considerations of absorbing boundary condition and boundary conditions at imperfectly conducting surfaces $[14,37]$.

The last two conditions introduced in this subsection are expressed in terms of the constitutive parameters of the media involved and of the velocity field. In particular, the first of these is:

HMV4. the fields $\mu_{r}, \varepsilon_{r}$ and $\beta$ allow to find $C_{m v 4}>0$ such that $\frac{1-\mu_{r} \varepsilon_{r} \beta^{2}}{\mu_{r}\left(1-\beta^{2}\right)} \geq C_{m v 4}$ in $\Omega_{k}, \forall k \in M_{\beta}$.

Remark 4.12. Where the media are at rest we have $\frac{\zeta_{1}}{\mu_{r}}=\frac{1-\mu_{r} \varepsilon_{r} \beta^{2}}{\mu_{r}\left(1-\beta^{2}\right)}=\frac{1}{\mu_{r}}$. Then, in these media $\operatorname{Re}\left(\frac{\zeta_{1}}{\mu_{r}}\right)=$ $\operatorname{Re}\left(\frac{1}{\mu_{r}}\right) \geq C_{\mu M}$ by HM9. In $\Omega_{k}, \forall k \in M_{\beta}, \frac{\zeta_{1}}{\mu_{r}}=\frac{1-\mu_{r} \varepsilon_{r} \beta^{2}}{\mu_{r}\left(1-\beta^{2}\right)}$ is real valued by HM3.

Before stating the last condition of interest in this subsection we define

$$
C_{m v 4 m}=\min \left(\left(\min _{k \in M_{0}} C_{\mu M k}\right), C_{m v 4}\right)
$$


and observe that, by using HM3, HM5 and HV4

$$
\exists C_{g l 2} \in \mathbb{R}:\left|\frac{\zeta_{2}}{\mu_{r}}\right| \leq C_{g l 2} \text { in } \Omega_{i}, \forall i \in M .
$$

Now the last hypothesis we require in this subsection reads

HMV5. the fields $\mu_{r}, \varepsilon_{r}$ and $\beta$ are such that $\left(1-\frac{C_{g l 2}^{2}}{C_{m v 3} C_{m v 4 m}}\right)>0$.

For the next developments it is also important to introduce a decomposition of $U_{2 D}$. In order to do so, let us consider the function space

$$
U_{2 D 0}=\left\{\mathbf{A}_{t} \in U_{2 D} \mid \operatorname{curl}_{2 D} \mathbf{A}_{t}=0 \text { and } \mathbf{A}_{t} \cdot \mathbf{l}=0\right\} .
$$

It is a closed subspace of $U_{2 D}$ and of $\left(L^{2}(\Omega)\right)^{2}[45]$.

The sesquilinear form $\left(\frac{1+\varepsilon_{r} \mu_{r}-\zeta_{1}}{\mu_{r}} \mathbf{u}_{t}, \mathbf{v}_{t}\right)_{0, \Omega}=\left(\frac{\mu_{r} \varepsilon_{r}-\beta^{2}}{\mu_{r}\left(1-\beta^{2}\right)} \mathbf{u}_{t}, \mathbf{v}_{t}\right)_{0, \Omega}$, defined on $\left(L^{2}(\Omega)\right)^{2} \times\left(L^{2}(\Omega)\right)^{2}$, is continuous if we assume HM3, HM5 and HV4. In particular, by assuming HM3, HM5 and HV4 we get

$$
\left|\frac{1+\varepsilon_{r} \mu_{r}-\zeta_{1}}{\mu_{r}}\right| \leq C_{l 4} \text { in } \Omega_{i}, \forall i \in M \text {. }
$$

The indicated sesquilinear form is coercive ([18], p. 20) whenever HMV3 is satisfied. As a matter of fact,

$$
\begin{aligned}
\left|\left(\frac{\mu_{r} \varepsilon_{r}-\beta^{2}}{\mu_{r}\left(1-\beta^{2}\right)} \mathbf{u}_{t}, \mathbf{u}_{t}\right)_{0, \Omega}\right|^{2} & =\left.\left.\left|\int_{\Omega} \frac{\mu_{r} \varepsilon_{r}-\beta^{2}}{\mu_{r}\left(1-\beta^{2}\right)}\right| \mathbf{u}_{t}\right|^{2}\right|^{2} \\
& =\left.\left|\int_{\Omega} \operatorname{Re}\left(\frac{\mu_{r} \varepsilon_{r}-\beta^{2}}{\mu_{r}\left(1-\beta^{2}\right)}\right)\right| \mathbf{u}_{t}\right|^{2}+\left.j \int_{\Omega} \operatorname{Im}\left(\frac{\mu_{r} \varepsilon_{r}-\beta^{2}}{\mu_{r}\left(1-\beta^{2}\right)}\right)\left|\mathbf{u}_{t}\right|^{2}\right|^{2} \\
& \geq\left.\left.\left|\int_{\Omega} \operatorname{Re}\left(\frac{\mu_{r} \varepsilon_{r}-\beta^{2}}{\mu_{r}\left(1-\beta^{2}\right)}\right)\right| \mathbf{u}_{t}\right|^{2}\right|^{2} \geq C_{m v 3}^{2}\left\|\mathbf{u}_{t}\right\|_{0, \Omega}^{4} .
\end{aligned}
$$

Then, under hypotheses HD1, HM3, HM5, HV4 and HMV3, we easily obtain the following Helmholtz decomposition ([18], Lem. 4.5, p. 86)

$$
U_{2 D}=U_{2 D 0} \oplus U_{2 D 1}
$$

where

$$
U_{2 D 1}=\left\{\mathbf{u}_{t} \in U_{2 D} \mid\left(\frac{1+\varepsilon_{r} \mu_{r}-\zeta_{1}}{\mu_{r}} \mathbf{u}_{t}, \mathbf{v}_{t}\right)_{0, \Omega}=0 \quad \forall \mathbf{v}_{t} \in U_{2 D 0}\right\}
$$

is a closed subspace of $U_{2 D}$.

The main conclusion of this subsection is given by

Theorem 4.13. Under the hypotheses HD1-HD3, HV1-HV4, HM1-HM6, HM8, HM9, HB1-HB2, HB3S, HMV1HMV5 the sesquilinear form a satisfies the so-called "inf-sup" condition ([18], p. 21)

$$
\text { we can find } \alpha: \inf _{\mathbf{u} \in U,\|\mathbf{u}\|_{U}=1} \sup _{\mathbf{v} \in U,\|\mathbf{v}\|_{U} \leq 1}|a(\mathbf{u}, \mathbf{v})| \geq \alpha>0 \text {. }
$$

We will prove Theorem 4.13 by contradiction, that is by assuming that under the indicated hypotheses condition (4.38) does not hold true. One can easily deduce that when (4.38) is not satisfied

$$
\exists\left\{\mathbf{u}_{n}\right\}, \mathbf{u}_{n} \in U \text { and }\left\|\mathbf{u}_{n}\right\|_{U}=1 \forall n \in \mathbb{N} \text {, such that } \lim _{n \rightarrow \infty} \sup _{\mathbf{v} \in U,\|\mathbf{v}\|_{U} \leq 1}\left|a\left(\mathbf{u}_{n}, \mathbf{v}\right)\right|=0 .
$$

The proof is split into several parts. Most of these are proved with the following lemmas. 
Lemma 4.14. Under the hypotheses HD1-HD3, HV1-HV4, HM1-HM6, HM8, HB1-HB2, HMV1, HMV2, HMV3 and HM7 or HB3, for any sequence $\left\{\mathbf{u}_{n}\right\}$ satisfying (4.39) we can find, on a common subsequence of indeces, $\left\{\mathbf{u}_{n t 0}\right\}$ weakly converging to zero in $U_{2 D},\left\{u_{n z}\right\}$ weakly converging to zero in $H^{1}(\Omega)$ and strongly converging to zero in $L^{2}(\Omega)$, and $\left\{\mathbf{u}_{n t 1}\right\}$ weakly converging to zero in $U_{2 D}$ and strongly converging to zero in $\left(L^{2}(\Omega)\right)^{2}$.

Proof. Let us consider a sequence $\left\{\mathbf{u}_{n}\right\}$ satisfying (4.39) and the sesquilinear form $a$ defined in (3.11). In Section 3 we deduced that under hypotheses HM3, HM5, HB1 and HV4 the sesquilinear form $a$ is bounded. Then, for any given $\mathbf{v} \in U$ we have that $a(\mathbf{u}, \mathbf{v})$ define a linear and bounded functional on $U$. By a classical property of Hilbert spaces ([38], pp. 287, 289 and 303), $\left\{\mathbf{u}_{n}\right\}$ contains a subsequence (still denoted by $\left\{\mathbf{u}_{n}\right\}$ ) weakly converging in $U$ to $\tilde{\mathbf{u}} \in U$, so that

$$
\forall \mathbf{v} \in U: \lim _{n \rightarrow \infty} a\left(\mathbf{u}_{n}, \mathbf{v}\right)=a(\tilde{\mathbf{u}}, \mathbf{v}) .
$$

One can wonder if $\tilde{\mathbf{u}}$ could be different from zero. This is not possible, however, under the additional hypotheses which guarantee uniqueness of the solution. As a matter of fact, under these hypotheses we have, for $\tilde{\mathbf{u}} \neq 0$

$$
\exists \tilde{\mathbf{v}} \in U \text { such that } a(\tilde{\mathbf{u}}, \tilde{\mathbf{v}}) \neq 0
$$

and, by (4.40)

$$
\lim _{n \rightarrow \infty} a\left(\mathbf{u}_{n}, \tilde{\mathbf{v}}\right) \neq 0
$$

which is at odds with (4.39). Then, under the hypotheses HD1-HD3, HV1-HV4, HM1-HM6, HM8, HB1-HB2, HMV1, HMV2, and HM7 or HB3, for any sequence $\left\{\mathbf{u}_{n}\right\}$ satisfying (4.39) we can find a subsequence weakly converging to zero in $U$, that is the subsequence $\left\{\mathbf{u}_{n t}\right\}$ weakly converges to zero in $U_{2 D}$ and $\left\{u_{n z}\right\}$ weakly converges to zero in $H^{1}(\Omega)$.

Under hypotheses HD1 and HD2 it is very well-known ([26], pp. 5 and 7$)$ that $H^{1}(\Omega)$ is compactly imbedded in $L^{2}(\Omega)$. Thus, from the previous subsequence of $\left\{u_{n z}\right\}$ we can extract another subsequence which converges strongly in $L^{2}(\Omega)$ to $\hat{u}_{z}$. Since both weak convergence in $H^{1}(\Omega)$ and strong convergence in $L^{2}(\Omega)$ imply weak convergence in $L^{2}(\Omega)$ to the same limit, we immediately deduce $\hat{u}_{z}=0$.

A similar result can be achieved on a part of $\left\{\mathbf{u}_{n t}\right\}$. As a matter of fact, by using hypotheses HD1, HD2, HM3, HM5, HV4 and HMV3 one can easily prove that $U_{2 D 1}$ is compactly imbedded in $\left(L^{2}(\Omega)\right)^{2}([18]$, pp. 8788, [45]). But by using the Helmholtz decomposition (4.36) and the inequalities (4.34) and (4.35), from any sequence $\left\{\mathbf{u}_{n}\right\}$ satisfying condition (4.39) we obtain two bounded sequences $\left\{\mathbf{u}_{n t 0}\right\},\left\{\mathbf{u}_{n t 1}\right\}, \mathbf{u}_{n t 0} \in U_{2 D 0}$, $\mathbf{u}_{n t 1} \in U_{2 D 1},\left\|\mathbf{u}_{n t 0}\right\|_{U_{2 D}} \leq \frac{C_{l 4}}{C_{m v 3}},\left\|\mathbf{u}_{n t 1}\right\|_{U_{2 D}} \leq \frac{C_{m v 3}+C_{l 4}}{C_{m v 3}}$ for all $n \in \mathbb{N}$. Then, from $\left\{\mathbf{u}_{n t 1}\right\}$ we can extract a subsequence which converges strongly in $\left(L^{2}(\Omega)\right)^{2}$ to $\hat{\mathbf{u}}_{t 1}$. But we know that $\left\{\mathbf{u}_{n t}\right\}$ weakly converges to zero in $U_{2 D}$ and this implies that both $\left\{\mathbf{u}_{n t 0}\right\}$ and $\left\{\mathbf{u}_{n t 1}\right\}$ weakly converge to zero in $U_{2 D}$. Now, since both weak convergence in $U_{2 D}$ and strong convergence in $\left(L^{2}(\Omega)\right)^{2}$ imply weak convergence in $\left(L^{2}(\Omega)\right)^{2}$ to the same limit, we immediately deduce $\hat{\mathbf{u}}_{t 1}=0$.

Another property of any sequence $\left\{\mathbf{u}_{n}\right\}$ satisfying (4.39) is given by the next lemma.

Lemma 4.15. Under the hypotheses HD1-HD3, HV1-HV4, HM1-HM6, HM8, HM9, HB1-HB2, HMV1, HMV2, HMV3 and HB3S, we deduce that for any sequence $\left\{\mathbf{u}_{n}\right\}$ satisfying (4.39) we can find a subsequence $\left\{\mathbf{u}_{n t 1}\right\}$ strongly converging to zero in $U_{2 D}$.

Proof. By using $\mathbf{v} \in U$ such that $\mathbf{v}_{t}=\mathbf{u}_{n t 1}, v_{z}=0$, from (3.11), (4.36) and (4.33) we deduce, for any $n$ of the sequence

$$
\begin{aligned}
& a\left(\mathbf{u}_{n},\left(\mathbf{u}_{n t 1}, 0\right)\right)=\left(\frac{1}{\mu_{r}} \operatorname{curl}_{2 D} \mathbf{u}_{n t 1}, \operatorname{curl}_{2 D} \mathbf{u}_{n t 1}\right)_{0, \Omega} \\
& -j \frac{\omega}{c_{0}}\left(\frac{\zeta_{2}}{\mu_{r}} \operatorname{grad}_{2 D} u_{n z}, \mathbf{u}_{n t 1}\right)_{0, \Omega}-\frac{\omega^{2}}{c_{0}^{2}}\left(\frac{1+\varepsilon_{r} \mu_{r}-\zeta_{1}}{\mu_{r}} \mathbf{u}_{n t}, \mathbf{u}_{n t 1}\right)_{0, \Omega} \\
& +j \omega \mu_{0}\left(Y\left(\mathbf{u}_{n t 1} \cdot \mathbf{l}\right), \mathbf{u}_{n t 1} \cdot \mathbf{l}\right)_{0, \Gamma} .
\end{aligned}
$$


Then

$$
\begin{aligned}
& C_{\mu M}\left\|\operatorname{curl}_{2 D} \mathbf{u}_{n t 1}\right\|_{0, \Omega}^{2} \leq\left|\left(\frac{1}{\mu_{r}} \operatorname{curl}_{2 D} \mathbf{u}_{n t 1}, \operatorname{curl}_{2 D} \mathbf{u}_{n t 1}\right)_{0, \Omega}\right| \\
& \leq\left|a\left(\mathbf{u}_{n},\left(\mathbf{u}_{n t 1}, 0\right)\right)\right|+\left|\frac{\omega}{c_{0}}\left(\frac{\zeta_{2}}{\mu_{r}} \operatorname{grad}_{2 D} u_{n z}, \mathbf{u}_{n t 1}\right)_{0, \Omega}\right| \\
& +\left|\frac{\omega^{2}}{c_{0}^{2}}\left(\frac{1+\varepsilon_{r} \mu_{r}-\zeta_{1}}{\mu_{r}} \mathbf{u}_{n t}, \mathbf{u}_{n t 1}\right)_{0, \Omega}\right|+\left|\omega \mu_{0}\left(Y\left(\mathbf{u}_{n t 1} \cdot \mathbf{l}\right), \mathbf{u}_{n t 1} \cdot \mathbf{l}\right)_{0, \Gamma}\right|
\end{aligned}
$$

Taking account that our hypotheses guarantee the validity of (4.32) and that, by HB1, we have

$$
|Y| \leq C_{Y M} \text { on } \Gamma
$$

we deduce

$$
\begin{aligned}
& C_{\mu M}\left\|\operatorname{curl}_{2 D} \mathbf{u}_{n t 1}\right\|_{0, \Omega}^{2} \\
& \leq\left|a\left(\mathbf{u}_{n},\left(\mathbf{u}_{n t 1}, 0\right)\right)\right|+\frac{\omega}{c_{0}} C_{g l 2}\left\|\operatorname{grad}_{2 D} u_{n z}\right\|_{0, \Omega}\left\|\mathbf{u}_{n t 1}\right\|_{0, \Omega} \\
& +\frac{\omega^{2}}{c_{0}^{2}} C_{l 4}\left\|\mathbf{u}_{n t}\right\|_{0, \Omega}\left\|\mathbf{u}_{n t 1}\right\|_{0, \Omega}+\omega \mu_{0} C_{Y M}\left\|\mathbf{u}_{n t} \cdot \mathbf{l}\right\|_{0, \Gamma}^{2} .
\end{aligned}
$$

But under hypothesis HB3S, HM6 and HV4 we immediately obtain

$$
\begin{aligned}
& \left|a\left(\mathbf{u}_{n}, \mathbf{u}_{n}\right)\right| \\
& \quad \geq \omega \mu_{0}\left(\operatorname{Re}(Y)\left(\gamma_{0} u_{n z}\right), \gamma_{0} u_{n z}\right)_{0, \Gamma}+\omega \mu_{0}\left(\operatorname{Re}(Y)\left(\mathbf{u}_{n t} \cdot \mathbf{l}\right), \mathbf{u}_{n t} \cdot \mathbf{l}\right)_{0, \Gamma} \\
& \geq \omega \mu_{0} C_{Y m}\left(\left\|\gamma_{0} u_{n z}\right\|_{0, \Gamma}^{2}+\left\|\mathbf{u}_{n t} \cdot \mathbf{l}\right\|_{0, \Gamma}^{2}\right)
\end{aligned}
$$

so that, since $\omega>0$ and $C_{Y m}>0$, for any sequence $\left\{\mathbf{u}_{n}\right\}$ satisfying (4.39)

$$
\lim _{n \rightarrow \infty}\left\|\gamma_{0} u_{n z}\right\|_{0, \Gamma}=0
$$

and

$$
\lim _{n \rightarrow \infty}\left\|\mathbf{u}_{n t} \cdot \mathbf{l}\right\|_{0, \Gamma}=0 .
$$

If we now consider the terms on the right-hand side of inequality (4.46) we can easily verify that all of them converge to zero on a subsequence. As a matter of fact, $\left\{\mathbf{u}_{n}\right\}$ satisfies (4.39), $\left\{\mathbf{u}_{n t 1}\right\}$ is bounded in $U_{2 D}$ and then $\left|a\left(\mathbf{u}_{n},\left(\mathbf{u}_{n t 1}, 0\right)\right)\right| \rightarrow 0$ as $n \rightarrow \infty$; moreover, $\left\|\mathbf{u}_{n}\right\|_{U}=1$ so that $\left\|\operatorname{grad}_{2 D} u_{n z}\right\|_{0, \Omega} \leq 1$ and $\left\|\mathbf{u}_{n t}\right\|_{0, \Omega} \leq 1$; since we know that $\left\|\mathbf{u}_{n t 1}\right\|_{0, \Omega} \rightarrow 0$ as $n \rightarrow \infty$ on the indicated subsequence the second and the third addend on the right-hand side of inequality (4.46) becomes smaller and smaller; finally, by (4.49), the fourth addend goes to zero. Then, by inequality (4.46) we deduce that, for any $\left\{\mathbf{u}_{n}\right\}$ satisfying (4.39), we have $\left\|\operatorname{curl}_{2 D} \mathbf{u}_{n t 1}\right\|_{0, \Omega}^{2} \rightarrow 0$ as $n \rightarrow \infty$ on a subsequence.

At the moment the deduced properties for $\left\{\mathbf{u}_{n t 0}\right\}$ and $\left\{\operatorname{grad}_{2 D} u_{n z}\right\}$ are not enough for our purpose. To overcome this difficulty, we state the third lemma of this subsection.

Lemma 4.16. Under the hypotheses HD1-HD3, HV1-HV4, HM1-HM6, HM8, HM9, HB1-HB2, HMV1, HMV2, HMV3, HB3S and HMV4 we have that for any sequence $\left\{\mathbf{u}_{n}\right\}$ satisfying (4.39) we can find a subsequence such that $\left\|\mathbf{u}_{n t 0}\right\|_{0, \Omega} \rightarrow 0$ as $n \rightarrow \infty$ if and only if $\left\|\operatorname{grad}_{2 D} u_{n z}\right\|_{0, \Omega} \rightarrow 0$ as $n \rightarrow \infty$. 
Proof. Let us consider $\mathbf{v} \in U$ such that $\mathbf{v}_{t}=\mathbf{u}_{n t 0}, v_{z}=0$. From (3.11) and (4.33) we deduce, for any $n$ of the sequence

$$
a\left(\mathbf{u}_{n},\left(\mathbf{u}_{n t 0}, 0\right)\right)=-j \frac{\omega}{c_{0}}\left(\frac{\zeta_{2}}{\mu_{r}} \operatorname{grad}_{2 D} u_{n z}, \mathbf{u}_{n t 0}\right)_{0, \Omega}-\frac{\omega^{2}}{c_{0}^{2}}\left(\frac{1+\varepsilon_{r} \mu_{r}-\zeta_{1}}{\mu_{r}} \mathbf{u}_{n t}, \mathbf{u}_{n t 0}\right)_{0, \Omega},
$$

so that, by considering (4.36) and (4.37)

$$
\begin{aligned}
C_{m v 3}\left\|\mathbf{u}_{n t 0}\right\|_{0, \Omega}^{2} & \leq\left|\left(\frac{1+\varepsilon_{r} \mu_{r}-\zeta_{1}}{\mu_{r}} \mathbf{u}_{n t 0}, \mathbf{u}_{n t 0}\right)_{0, \Omega}\right| \\
& \leq \frac{c_{0}^{2}}{\omega^{2}}\left|a\left(\mathbf{u}_{n},\left(\mathbf{u}_{n t 0}, 0\right)\right)\right|+\frac{c_{0}}{\omega}\left|\left(\frac{\zeta_{2}}{\mu_{r}} \operatorname{grad}_{2 D} u_{n z}, \mathbf{u}_{n t 0}\right)_{0, \Omega}\right| \\
& \leq \frac{c_{0}^{2}}{\omega^{2}}\left|a\left(\mathbf{u}_{n},\left(\mathbf{u}_{n t 0}, 0\right)\right)\right|+\frac{c_{0}}{\omega} C_{g l 2}\left\|\operatorname{grad}_{2 D} u_{n z}\right\|_{0, \Omega}\left\|\mathbf{u}_{n t 0}\right\|_{0, \Omega} .
\end{aligned}
$$

Then, since $\left\{\mathbf{u}_{n t 0}\right\}$ is bounded in $U_{2 D}$ and then also in $\left(L^{2}(\Omega)\right)^{2}$, for any sequence $\left\{\mathbf{u}_{n}\right\}$ satisfying (4.39) we have that $\left\|\operatorname{grad}_{2 D} u_{n z}\right\|_{0, \Omega} \rightarrow 0$ on any subsequence implies $\left\|\mathbf{u}_{n t 0}\right\|_{0, \Omega} \rightarrow 0$ on the same subsequence. It is possible to show that the opposite implication holds true as well. As a matter of fact, we consider $\mathbf{v} \in U$ such that $\mathbf{v}_{t}=\mathbf{0}$ and $v_{z}=u_{n z}$. From (3.11) we deduce, for any $n$ of the sequence

$$
\begin{aligned}
a\left(\mathbf{u}_{n},\left(\mathbf{0}, u_{n z}\right)\right)= & \left(\frac{\zeta_{1}}{\mu_{r}} \operatorname{grad}_{2 D} u_{n z}, \operatorname{grad}_{2 D} u_{n z}\right)_{0, \Omega} \\
& +j \frac{\omega}{c_{0}}\left(\frac{\zeta_{2}}{\mu_{r}} \mathbf{u}_{n t}, \operatorname{grad}_{2 D} u_{n z}\right)_{0, \Omega}-\frac{\omega^{2}}{c_{0}^{2}}\left(\varepsilon_{r} u_{n z}, u_{n z}\right)_{0, \Omega}+j \omega \mu_{0}\left(Y\left(\gamma_{0} u_{n z}\right), \gamma_{0} u_{n z}\right)_{0, \Gamma} .
\end{aligned}
$$

From (4.31) we get

$$
\begin{aligned}
C_{m v 4 m}\left\|\operatorname{grad}_{2 D} u_{n z}\right\|_{0, \Omega}^{2} \leq & \left|\left(\frac{\zeta_{1}}{\mu_{r}} \operatorname{grad}_{2 D} u_{n z}, \operatorname{grad}_{2 D} u_{n z}\right)_{0, \Omega}\right| \\
\leq & \left|a\left(\mathbf{u}_{n},\left(\mathbf{0}, u_{n z}\right)\right)\right|+\frac{\omega}{c_{0}}\left|\left(\frac{\zeta_{2}}{\mu_{r}}\left(\mathbf{u}_{n t 0}+\mathbf{u}_{n t 1}\right), \operatorname{grad}_{2 D} u_{n z}\right)_{0, \Omega}\right| \\
& +\frac{\omega^{2}}{c_{0}^{2}}\left|\left(\varepsilon_{r} u_{n z}, u_{n z}\right)_{0, \Omega}\right|+\omega \mu_{0}\left|\left(Y\left(\gamma_{0} u_{n z}\right), \gamma_{0} u_{n z}\right)_{0, \Gamma}\right| \\
\leq & \left|a\left(\mathbf{u}_{n},\left(\mathbf{0}, u_{n z}\right)\right)\right|+\frac{\omega}{c_{0}} C_{g l 2}\left\|\mathbf{u}_{n t 0}\right\|_{0, \Omega}\left\|\operatorname{grad}_{2 D} u_{n z}\right\|_{0, \Omega} \\
& +\frac{\omega}{c_{0}} C_{g l 2}\left\|\mathbf{u}_{n t 1}\right\|_{0, \Omega}\left\|\operatorname{grad}_{2 D} u_{n z}\right\|_{0, \Omega}+\frac{\omega^{2}}{c_{0}^{2}} C_{\varepsilon M}\left\|u_{n z}\right\|_{0, \Omega}^{2}+\omega \mu_{0} C_{Y M}\left\|\gamma_{0} u_{n z}\right\|_{0, \Gamma}^{2},
\end{aligned}
$$

where, by HM3, we can find $C_{\varepsilon M}$ such that $\left|\varepsilon_{r}\right| \leq C_{\varepsilon M}$ in $\Omega_{i}, \forall i \in M$. By using (4.48), the strong convergence to zero in $L^{2}(\Omega)$ of a subsequence of $\left\{u_{n z}\right\}$, the strong convergence to zero in $U_{2 D}$ of the same subsequence of $\left\{\mathbf{u}_{n t 1}\right\}$ and the convergence to zero of $\left|a\left(\mathbf{u}_{n},\left(\mathbf{0}, u_{n z}\right)\right)\right|$, we deduce that $\left\|\mathbf{u}_{n t 0}\right\|_{0, \Omega} \rightarrow 0$ implies $\left\|\operatorname{grad}_{2 D} u_{n z}\right\|_{0, \Omega} \rightarrow 0$ on the same subsequence.

With these results it is easy to complete the proof of Theorem 4.13. As a matter of fact, the set of hypotheses considered in the statement of the theorem contains those considered in all the above lemmas and we can use all their conclusions for any sequence $\left\{\mathbf{u}_{n}\right\}$ satisfying (4.39). Let us now consider a subsequence having 
all properties guaranteed by Lemmas 4.14-4.16. Suppose that both $\left\|\mathbf{u}_{n t 0}\right\|_{0, \Omega} \rightarrow 0$ and $\left\|\operatorname{grad}_{2 D} u_{n z}\right\|_{0, \Omega} \rightarrow 0$ as $n \rightarrow \infty$ on the indicated subsequence. By taking account also of (4.48), we immediately deduce that the indicated subsequence of $\left\{\mathbf{u}_{n}\right\}$ converges strongly to zero in $U$ and this is at odds with (4.39). Then, we deduce that on the indicated subsequence (giving the strong convergence to zero in $L^{2}(\Omega)$ of $\left\{u_{n z}\right\}$ and the strong convergence to zero in $U_{2 D}$ of $\left.\left\{\mathbf{u}_{n t 1}\right\}\right)$ both $\left\{\mathbf{u}_{n t 0}\right\}$ and $\left\{\operatorname{grad}_{2 D} u_{n z}\right\}$ do not converge to zero in $\left(L^{2}(\Omega)\right)^{2}$.

It is then possible to find $\epsilon>0$ such that for any $\tilde{n} \in \mathbb{N}$ there exist an integer $n \geq \tilde{n}$ such that $\left\|\mathbf{u}_{n t 0}\right\|_{0, \Omega} \geq \epsilon$. This permits to define a subsequence of the previous subsequence guaranteeing strong convergence to zero in $L^{2}(\Omega)$ of $\left\{u_{n z}\right\}$, strong convergence to zero in $U_{2 D}$ of $\left\{\mathbf{u}_{n t 1}\right\}$ and such that $\left\|\mathbf{u}_{n t 0}\right\|_{0, \Omega} \geq \epsilon$ for any $n$ of this subsequence.

On this subsequence, from (4.51) we obtain

$$
C_{m v 3}\left\|\mathbf{u}_{n t 0}\right\|_{0, \Omega} \leq \frac{c_{0}^{2}}{\omega^{2}}\left|a\left(\mathbf{u}_{n},\left(\frac{\mathbf{u}_{n t 0}}{\left\|\mathbf{u}_{n t 0}\right\|_{0, \Omega}}, 0\right)\right)\right|+\frac{c_{0}}{\omega} C_{g l 2}\left\|\operatorname{grad}_{2 D} u_{n z}\right\|_{0, \Omega} .
$$

Let us substitute this result in inequality (4.53)

$$
\begin{aligned}
\left\|\operatorname{grad}_{2 D} u_{n z}\right\|_{0, \Omega}^{2} \leq & \frac{1}{C_{m v 4 m}}\left|a\left(\mathbf{u}_{n},\left(\mathbf{0}, u_{n z}\right)\right)\right|+\frac{\omega C_{g l 2}}{c_{0} C_{m v 4 m}}\left\|\operatorname{grad}_{2 D} u_{n z}\right\|_{0, \Omega} \\
& \times\left(\frac{c_{0}^{2}}{\omega^{2} C_{m v 3}}\left|a\left(\mathbf{u}_{n},\left(\frac{\mathbf{u}_{n t 0}}{\left\|\mathbf{u}_{n t 0}\right\|_{0, \Omega}}, 0\right)\right)\right|+\frac{c_{0} C_{g l 2}}{\omega C_{m v 3}}\left\|\operatorname{grad}_{2 D} u_{n z}\right\|_{0, \Omega}\right) \\
& +\frac{\omega C_{g l 2}}{c_{0} C_{m v 4 m}}\left\|\mathbf{u}_{n t 1}\right\|_{0, \Omega}\left\|\operatorname{grad}_{2 D} u_{n z}\right\|_{0, \Omega}+\frac{\omega^{2} C_{\varepsilon M}}{c_{0}^{2} C_{m v 4 m}}\left\|u_{n z}\right\|_{0, \Omega}^{2}+\frac{\omega \mu_{0} C_{Y M}}{C_{m v 4 m}}\left\|\gamma_{0} u_{n z}\right\|_{0, \Gamma}^{2} \\
= & \frac{1}{C_{m v 4 m}}\left|a\left(\mathbf{u}_{n},\left(\mathbf{0}, u_{n z}\right)\right)\right|+\frac{c_{0} C_{g l 2}}{\omega C_{m v 3} C_{m v 4 m}}\left\|\operatorname{grad}_{2 D} u_{n z}\right\|_{0, \Omega}\left|a\left(\mathbf{u}_{n},\left(\frac{\mathbf{u}_{n t 0}}{\left\|\mathbf{u}_{n t 0}\right\|_{0, \Omega}}, 0\right)\right)\right| \\
& +\frac{C_{g l 2}^{2}}{C_{m v 3} C_{m v 4 m}}\left\|\operatorname{grad}_{2 D} u_{n z}\right\|_{0, \Omega}^{2} \\
& +\frac{\omega C_{g l 2}}{c_{0} C_{m v 4 m}}\left\|\mathbf{u}_{n t 1}\right\|_{0, \Omega}\left\|\operatorname{grad}_{2 D} u_{n z}\right\|_{0, \Omega}+\frac{\omega^{2} C_{\varepsilon M}}{c_{0}^{2} C_{m v 4 m}}\left\|u_{n z}\right\|_{0, \Omega}^{2}+\frac{\omega \mu_{0} C_{Y M}}{C_{m v 4 m}}\left\|\gamma_{0} u_{n z}\right\|_{0, \Gamma}^{2} .
\end{aligned}
$$

Then

$$
\begin{aligned}
& \left(1-\frac{C_{g l 2}^{2}}{C_{m v 3} C_{m v 4 m}}\right)\left\|\operatorname{grad}_{2 D} u_{n z}\right\|_{0, \Omega}^{2} \\
& \leq \frac{1}{C_{m v 4 m}}\left|a\left(\mathbf{u}_{n},\left(\mathbf{0}, u_{n z}\right)\right)\right|+\frac{c_{0} C_{g l 2}}{\omega C_{m v 3} C_{m v 4 m}}\left\|\operatorname{grad}_{2 D} u_{n z}\right\|_{0, \Omega}\left|a\left(\mathbf{u}_{n},\left(\frac{\mathbf{u}_{n t 0}}{\left\|\mathbf{u}_{n t 0}\right\|_{0, \Omega}}, 0\right)\right)\right| \\
& +\frac{\omega C_{g l 2}}{c_{0} C_{m v 4 m}}\left\|\mathbf{u}_{n t 1}\right\|_{0, \Omega}\left\|\operatorname{grad}_{2 D} u_{n z}\right\|_{0, \Omega}+\frac{\omega^{2} C_{\varepsilon M}}{c_{0}^{2} C_{m v 4 m}}\left\|u_{n z}\right\|_{0, \Omega}^{2}+\frac{\omega \mu_{0} C_{Y M}}{C_{m v 4 m}}\left\|\gamma_{0} u_{n z}\right\|_{0, \Gamma}^{2} .
\end{aligned}
$$

Since the right-hand side goes to zero on the indicated subsequence, by using HMV5 we again conclude $\left\|\operatorname{grad}_{2 D} u_{n z}\right\|_{0, \Omega}^{2} \rightarrow 0$ as $n \rightarrow \infty$ and this is at odds with our starting hypothesis.

This completes the proof of Theorem 4.13 since there are no other possibilities to consider.

We are now in a position to state the main Theorem of this section.

Theorem 4.17. Under the hypotheses HD1-HD3, HV1-HV4, HM1-HM6, HM8, HM9, HB1-HB2, HB3S, HMV1HMV5 Problem 2 is well posed.

Proof. Under the indicated hypotheses the sesquilinear form involved in Problem 2 is bounded. Moreover, it satisfies (4.30). Finally, condition (4.38) is satisfied as well. Thus, we can apply the generalized Lax-Milgram lemma ([18], p. 21) to obtain the result of interest. 


\section{Galerkin approximation}

In order to approximate Problem 2 Galerkin's method ([46], p. 59) is widely adopted. In this method Problem 2 is substituted by a similar problem, the so-called discrete problem. It is posed in a finite dimensional subspace $U_{h}$ of $U$.

Any satisfactory approximation should at least guarantee convergence ([46], p. 112). Such a property is defined in terms of sequences of solutions of the discrete problems. These sequences of solutions are obtained by considering sequences $\left\{U_{h}\right\}, h \in I$, of finite dimensional subspaces of $U$, where the set $I$ is a denumerable and bounded set of strictly positive indexes having zero as the only limit point ([46], p. 112).

For any $h \in I$ and for any set of approximate sources $\mathbf{J}_{e h}, \mathbf{J}_{m h} \in\left(L^{2}(\Omega)\right)^{3}$ and $f_{R l h}, f_{R z h} \in L^{2}(\Gamma)$ we define the antilinear form

$$
\begin{aligned}
l_{h}(\mathbf{w})= & -j \omega \mu_{0}\left(J_{e z h}, w_{z}\right)_{0, \Omega}-\left(\frac{\zeta_{1}}{\mu_{r}} \hat{\mathbf{z}} \times \mathbf{J}_{m t h}, \operatorname{grad}_{2 D} w_{z}\right)_{0, \Omega}-j \omega \mu_{0}\left(f_{R z h}, \gamma_{0} w_{z}\right)_{0, \Gamma} \\
& -\left(\frac{1}{\mu_{r}} J_{m z h}, \operatorname{curl}_{2 D} \mathbf{w}_{t}\right)_{0, \Omega}-j \omega \mu_{0}\left(\mathbf{J}_{e t h}, \mathbf{w}_{t}\right)_{0, \Omega}+j \frac{\omega}{c_{0}}\left(\frac{\zeta_{2}}{\mu_{r}} \hat{\mathbf{z}} \times \mathbf{J}_{m t h}, \mathbf{w}_{t}\right)_{0, \Omega} \\
& -j \omega \mu_{0}\left(f_{R l h}, \mathbf{w}_{t} \cdot \mathbf{l}\right)_{0, \Gamma} \mathbf{w} \in U .
\end{aligned}
$$

Then, the aforementioned discrete version of Problem 2 reads as follows

Problem 4. Under the hypotheses HD1-HD3, HV1-HV4, HM1-HM5, HB1, given $\omega>0, \mathbf{J}_{e h}, \mathbf{J}_{m h} \in\left(L^{2}(\Omega)\right)^{3}$ and $f_{R l h}, f_{R z h} \in L^{2}(\Gamma)$, find $\mathbf{E}_{h} \in U_{h}$ such that

$$
a\left(\mathbf{E}_{h}, \mathbf{w}_{h}\right)=l_{h}\left(\mathbf{w}_{h}\right) \quad \forall \mathbf{w}_{h} \in U_{h} .
$$

Remark 5.1. In order to avoid additional difficulties in this work we assume that the sesquilinear form used in the definition of Problem 4 is identical to that of Problem 2 ([46], p. 183). By the same token, the weights appearing in the $\left(L^{2}(\Omega)\right)^{2}$ scalar products defining $l_{h}$ are identical to those used in the definition of $l$.

At this point one usually has to prove that for sufficiently small values of $h$ (that is $\exists h_{0}>0$ such that $\forall h \in I$, $h<h_{0}$ ([18], p. 169)) the solution of the discrete problem is unique. We skip this part, however, and, under some additional hypotheses related to the sequence $\left\{U_{h}\right\}$ of approximating spaces, we prove that the so-called discrete inf-sup condition holds uniformly in $h$ for the sesquilinear form $a$, that is

$$
\exists \exists \alpha, h_{0} \text { such that } \forall h \in I, h<h_{0}, \inf _{\mathbf{u}_{h} \in U_{h},\left\|\mathbf{u}_{h}\right\|_{U}=1} \sup _{\mathbf{v}_{h} \in U_{h},\left\|\mathbf{v}_{h}\right\|_{U} \leq 1}\left|a\left(\mathbf{u}_{h}, \mathbf{v}_{h}\right)\right| \geq \alpha>0 .
$$

In order to obtain this result, we need to introduce some additional notations and conditions. In particular, we define

$$
\begin{aligned}
U_{2 D h} & =\left\{\mathbf{u}_{h t} \mid \mathbf{u}_{h} \in U_{h}\right\} \subset U_{2 D}, \\
U_{h z} & =\left\{u_{h z} \mid \mathbf{u}_{h} \in U_{h}\right\} \subset H^{1}(\Omega) .
\end{aligned}
$$

For the subspace $U_{2 D h}$, under hypotheses HD1, HM3, HM5, HV4 and HMV3, we easily obtain the Helmholtz decomposition [45] corresponding to (4.36)

$$
U_{2 D h}=U_{2 D 0 h} \oplus U_{2 D 1 h}
$$

where

$$
U_{2 D 0 h}=\left\{\mathbf{A}_{h t} \in U_{2 D h} \mid \operatorname{curl}_{2 D} \mathbf{A}_{h t}=0 \text { and } \mathbf{A}_{h t} \cdot \mathbf{l}=0\right\} \subset U_{2 D 0}
$$

and

$$
U_{2 D 1 h}=\left\{\mathbf{u}_{h t} \in U_{2 D h} \mid\left(\frac{1+\varepsilon_{r} \mu_{r}-\zeta_{1}}{\mu_{r}} \mathbf{u}_{h t}, \mathbf{v}_{h t}\right)_{0, \Omega}=0 \quad \forall \mathbf{v}_{h t} \in U_{2 D 0 h}\right\} .
$$

We assume that the sequence $\left\{U_{h}\right\}$ of approximating spaces satisfies 
HSAS1. $\lim _{h \rightarrow 0} \inf _{\mathbf{u}_{h} \in U_{h}}\left\|\mathbf{u}-\mathbf{u}_{h}\right\|_{U}=0, \quad \forall \mathbf{u} \in U$, denoted by (CAS) in [45], and

HSAS2. from any subsequence of elements $\mathbf{u}_{h t} \in U_{2 D 1 h}$ which is bounded in $U_{2 D}$, one can extract a subsequence converging strongly in $\left(L^{2}(\Omega)\right)^{2}$ to an element of $U_{2 D}$,

denoted by (DCP) in [45] (see also [47-49]). We are now ready to state the result of interest related to the discrete inf-sup condition

Theorem 5.2. Under the hypotheses HD1-HD3, HV1-HV4, HM1-HM6, HM8, HM9, HB1-HB2, HB3S, HMV1HMV5, HSAS1 and HSAS2 the sesquilinear form a satisfies condition (5.3).

Proof. By contradiction, we assume it does not. Then, in analogy to what we have seen in Section 4.3, we know that

$$
\exists\left\{\mathbf{u}_{h}\right\}_{h \in J \subset I}, \mathbf{u}_{h} \in U_{h} \text { and }\left\|\mathbf{u}_{h}\right\|_{U}=1 \forall h \in J, \text { such that } \lim _{h \rightarrow 0} \sup _{\mathbf{v}_{h} \in U_{h},\left\|\mathbf{v}_{h}\right\|_{U} \leq 1}\left|a\left(\mathbf{u}_{h}, \mathbf{v}_{h}\right)\right|=0 .
$$

As in Section 4.3, taking account of its boundedness, we can find a subsequence of $\left\{\mathbf{u}_{h}\right\}_{h \in J}$ (still denoted by $\left\{\mathbf{u}_{h}\right\}_{h \in J}$ ) weakly converging in $U$ to $\tilde{\mathbf{u}} \in U$. If $\tilde{\mathbf{u}} \neq 0$, since our hypotheses guarantee uniqueness of the solution of the continuous problem, we can find, as in Section 4.3, $\tilde{\mathbf{v}} \in U$ such that $a(\tilde{\mathbf{u}}, \tilde{\mathbf{v}}) \neq 0$. Then, the weak convergence implies $\lim _{h \rightarrow 0} a\left(\mathbf{u}_{h}, \tilde{\mathbf{v}}\right) \neq 0$ (on the indicated subsequence). By using HSAS1 we can define $\left\{\mathbf{w}_{h}\right\}$ strongly converging in $U$ to $\tilde{\mathbf{v}}$ and then, by the continuity of the sesquilinear form, $C\left\|\mathbf{w}_{h}-\tilde{\mathbf{v}}\right\|_{U}=$ $C\left\|\mathbf{u}_{h}\right\|_{U}\left\|\mathbf{w}_{h}-\tilde{\mathbf{v}}\right\|_{U} \geq\left|a\left(\mathbf{u}_{h}, \mathbf{w}_{h}-\tilde{\mathbf{v}}\right)\right|=\left|a\left(\mathbf{u}_{h}, \mathbf{w}_{h}\right)-a\left(\mathbf{u}_{h}, \tilde{\mathbf{v}}\right)\right|$ and we see that the left-hand side goes to zero while the right-hand side does not (on the indicated subsequence). Thus $\tilde{\mathbf{u}} \neq 0$ is wrong and, then, $\tilde{\mathbf{u}}=0$.

The consequences of the compact imbeddings of Section 4.3 can be achieved in this case, too, as a consequence of (5.5) and of HSAS2 (the boundedness of the subsequence of $\mathbf{u}_{h t 1} \in U_{2 D 1 h}$ is deduced exactly as in Sect. 4.3). Then, as before, we can find a common subsequence of indeces such that $\left\{\mathbf{u}_{h z}\right\}$ and $\left\{\mathbf{u}_{h t 1}\right\}$ strongly converge to zero in $L^{2}(\Omega)$ and $\left(L^{2}(\Omega)\right)^{2}$, respectively.

Finally, all other considerations of Section 4.3 hold provided that we replace the subscript $n$ with $h$ everywhere, since all hypotheses considered in that subsection are assumed to hold true here, as well. In particular, equations (4.46) and (4.49) hold true with the indicated substitution and we deduce that we can find a subsequence $\left\{\mathbf{u}_{h t 1}\right\}$ strongly converging to zero in $U_{2 D}$. Equations (4.48), (4.50)-(4.56) hold true as well with the same substitution. Then on a subsequence we have that $\left\{\mathbf{u}_{h}\right\}$ converges strongly to zero in $U$ and this is at odds with (5.9). Thus condition (5.3) holds true for the sesquilinear form $a$.

One can immediately verify that condition (5.3) guarantees that the solution of the discrete problem is unique in the sense indicated above, that is $\exists h_{0}$ such that $\forall h \in I, h<h_{0}$, the solution of the discrete problem is unique. We denote by $I_{1}$ such a subset of $I$. Since $U_{h}$ is finite dimensional for all values of $h \in I$, from uniqueness we deduce existence of the solution of the discrete problem, under the same conditions.

From now on, under the hypotheses assumed in Theorem 5.2, we can talk of a uniquely defined sequence $\left\{\mathbf{E}_{h}\right\}_{h \in I_{1}}$ of solutions of the discrete problems, for any set of sequences $\left(\left\{\mathbf{J}_{e h}\right\},\left\{\mathbf{J}_{m h}\right\},\left\{f_{R l h}\right\},\left\{f_{R z h}\right\}\right), h \in I_{1}$.

Moreover, if we assume, in addition to the hypotheses of Theorem 5.2, that the sequences of discrete sources satisfy

HSDS1. $\left\{\mathbf{J}_{e h}\right\},\left\{\mathbf{J}_{m h}\right\}$ are bounded in $\left(L^{2}(\Omega)\right)^{2}$ and $\left\{f_{R l h}\right\},\left\{f_{R z h}\right\}$ are bounded in $L^{2}(\Gamma)$,

we easily conclude, again by contradiction, that the sequence $\left\{\mathbf{E}_{h}\right\}_{h \in I_{1}}$ of solutions of the discrete problems is bounded. If it was not we could define a subsequence satisfying condition (5.9) and this is at odds with the assumptions guaranteeing the validity of Theorem 5.2.

In order to consider Problem 4 as an approximation of Problem 2 one usually assumes that the sources of the discrete problem satisfy

HSDS2. $\lim _{h \rightarrow 0}\left\|\mathbf{J}_{e}-\mathbf{J}_{e h}\right\|_{0, \Omega}=0$, 
HSDS3. $\lim _{h \rightarrow 0}\left\|\mathbf{J}_{m}-\mathbf{J}_{m h}\right\|_{0, \Omega}=0$

HSDS4. $\lim _{h \rightarrow 0}\left\|\mathbf{f}_{R l}-\mathbf{f}_{R l h}\right\|_{0, \Gamma}=0$,

HSDS5. $\lim _{h \rightarrow 0}\left\|\mathbf{f}_{R z}-\mathbf{f}_{R z h}\right\|_{0, \Gamma}=0$,

so that HSDS1 is satisfied and, by the triangle and Cauchy-Schwartz inequalities, from (3.12) and (5.1) one gets

$$
\lim _{h \rightarrow 0} \sup _{\mathbf{w} \in U,\|\mathbf{w}\|_{U} \leq 1}\left|l(\mathbf{w})-l_{h}(\mathbf{w})\right|=0 .
$$

Thus, if we assume that all the hypotheses of Theorem 5.2 are satisfied and suppose, moreover, that HSDS2HSDS5 hold true for the considered sequences of discrete sources, we can consider the well defined and bounded (in $U$ ) sequence $\left\{\mathbf{E}-\mathbf{E}_{h}\right\}_{h \in I_{1}}$, where $\mathbf{E}$ is the solution of Problem 2 and $\left\{\mathbf{E}_{h}\right\}_{h \in I_{1}}$ is the sequence of solutions of Problem 4 for the indicated sequences of sources. As before, for the indicated boundedness in $U$, from the sequence $\left\{\mathbf{E}-\mathbf{E}_{h}\right\}$ it is possible to extract a subsequence which converges weakly in $U$ to $\tilde{\mathbf{u}} \in U$, so that

$$
\forall \mathbf{w} \in U: \lim _{h \rightarrow 0} a\left(\mathbf{E}-\mathbf{E}_{h}, \mathbf{w}\right)=a(\tilde{\mathbf{u}}, \mathbf{w}) .
$$

But by using (5.10), (3.13), (5.2) and $U_{h} \subset U, \forall h \in I$, one finds for $h \in I_{1}$

$$
\lim _{h \rightarrow 0} \sup _{\mathbf{w}_{h} \in U_{h},\left\|\mathbf{w}_{h}\right\|_{U} \leq 1}\left|a\left(\mathbf{E}, \mathbf{w}_{h}\right)-a\left(\mathbf{E}_{h}, \mathbf{w}_{h}\right)\right|=0 .
$$

Then, as in the proof of Theorem 5.2, by the uniqueness of the solution of the continuous problem, HSAS1 and the continuity of the sesquilinear form $a$, we immediately obtain that $\left\{\mathbf{E}-\mathbf{E}_{h}\right\}$ weakly converges to zero in $U$ on a subsequence.

If $\left\{\mathbf{E}-\mathbf{E}_{h}\right\}$ does not converge strongly in $U$ to zero, taking account of (5.11) with the right-hand side equal to zero and of (5.12), it is easy to deduce

$$
\begin{aligned}
& \exists \epsilon>0 \text { and a subsequence }\left\{\mathbf{E}-\mathbf{E}_{h}\right\}_{h \in J \subset I_{1}}, \text { with }\left\|\mathbf{E}-\mathbf{E}_{h}\right\|_{U}>\epsilon \quad \forall h \in J, \\
& \text { such that } \forall \mathbf{w} \in U: \lim _{h \rightarrow 0} \sup _{\mathbf{w}_{h} \in U_{h},\left\|\mathbf{w}_{h}\right\|_{U} \leq 1}\left|a\left(\mathbf{E}-\mathbf{E}_{h}, \mathbf{w}-\mathbf{w}_{h}\right)\right|=0 .
\end{aligned}
$$

This property allows us to repeat once more our procedure of Section 4.3 (see also the proof of Thm. 5.2). As a matter of fact, from the previous weak convergence it is easy to deduce that $\left\{E_{z}-E_{h z}\right\}$ weakly converges to zero in $H^{1}(\Omega)$ and $\left\{\mathbf{E}_{t}-\mathbf{E}_{h t}\right\}$ weakly converges to zero in $U_{2 D}$ on the same subsequence. Then, taking account of (5.5), we can find a subsequence of $\left\{E_{z}-E_{h z}\right\}$ strongly converging to zero in $L^{2}(\Omega)$. Moreover, by using the Helmholtz decompositions (4.36) and (5.6) we get, for any $h \in I_{1}, \mathbf{E}_{t}-\mathbf{E}_{h t}=\mathbf{E}_{t 0}+\mathbf{E}_{t 1}-\mathbf{E}_{h t 0}-\mathbf{E}_{h t 1}=$ $\left(\mathbf{E}_{t 0}-\mathbf{E}_{h t 0}\right)+\left(\mathbf{E}_{t 1}-\mathbf{E}_{h t 1}\right)$, where $\mathbf{E}_{t 0} \in U_{2 D 0}, \mathbf{E}_{h t 0} \in U_{2 D 0 h} \subset U_{2 D 0}, \mathbf{E}_{t 1} \in U_{2 D 1}$ and $\mathbf{E}_{h t 1} \in U_{2 D 1 h}$, so that if to the previous assumptions we add

HSAS3. $\lim _{h \rightarrow 0} \inf _{\mathbf{u}_{h t} \in U_{2 D 0 h}}\left\|\mathbf{u}-\mathbf{u}_{h t}\right\|_{U}=0, \quad \forall \mathbf{u} \in U_{2 D 0}$,

which was denoted by (CDK) in [45], we can deduce that, on a subsequence, $\left\{\mathbf{E}_{t 1}-\mathbf{E}_{h t 1}\right\}$ strongly converges to zero in $\left(L^{2}(\Omega)\right)^{2}[18,45]$. As a matter of fact, HSAS2 and HSAS3 imply $[18,45]$ that a subsequence of $\left\{\mathbf{E}_{t 1}-\mathbf{E}_{h t 1}\right\}$ strongly converges in $\left(L^{2}(\Omega)\right)^{2}$ to $\tilde{\mathbf{E}}_{t 1} \in U_{2 D 1}$. This deduction and the already proved weak convergence to zero in $U_{2 D}$ of $\left\{\mathbf{E}_{t}-\mathbf{E}_{h t}\right\}$ give the anticipated result.

Now, we can consider inequality (4.47) with $\mathbf{u}_{n}$ substituted by $\mathbf{E}-\mathbf{E}_{h}$ and deduce, by using (5.13) with $\mathbf{w}-\mathbf{w}_{h}$ replaced by $\mathbf{E}-\mathbf{E}_{h}$, the conditions corresponding to (4.48) and (4.49), that is

$$
\lim _{h \rightarrow 0}\left\|\gamma_{0}\left(E_{z}-E_{h z}\right)\right\|_{0, \Gamma}=0
$$

and

$$
\lim _{h \rightarrow 0}\left\|\left(\mathbf{E}_{t}-\mathbf{E}_{h t}\right) \cdot \mathbf{l}\right\|_{0, \Gamma}=0 .
$$


Analogously, from equation (4.43), with $\mathbf{u}_{n}$ and $\left(\mathbf{u}_{n t 1}, 0\right)$ substituted by $\mathbf{E}-\mathbf{E}_{h}$ and $\left(\mathbf{E}_{t 1}-\mathbf{E}_{h t 1}, 0\right)$, respectively, one gets (4.44) and (4.46) with the same substitutions, so that, taking account of (5.13) with $\mathbf{w}-\mathbf{w}_{h}$ substituted by $\left(\mathbf{E}_{t 1}-\mathbf{E}_{h t 1}, 0\right)$ and of (5.15) it is possible to deduce that one can find a subsequence of $\left\{\mathbf{E}_{t 1}-\mathbf{E}_{h t 1}\right\}_{h \in I_{1}}$ strongly converging to zero in $U_{2 D}$.

Finally, we consider equations (4.50) and (4.52), with $\mathbf{u}_{n}$ substituted by $\mathbf{E}-\mathbf{E}_{h}$. In the former we consider $\left(\mathbf{u}_{n t 0}, 0\right)$ replaced by $\left(\mathbf{E}_{t 0}-\mathbf{E}_{h t 0}, 0\right)$. It is easy to deduce, taking account of the strong convergence to zero of $\left\{\mathbf{E}_{t 1}-\mathbf{E}_{h t 1}\right\}_{h \in I_{1}}$ (in $\left(L^{2}(\Omega)\right)^{2}$ is enough), that inequality (4.51) holds true with the same substitutions. In (4.52) $\left(\mathbf{0}, E_{z}-E_{h z}\right)$ substitutes $\left(\mathbf{0}, u_{n z}\right)$ so that we obtain inequality (4.53), again with the same replacements. By using (5.13) with $\mathbf{w}-\mathbf{w}_{h}$ replaced by $\left(\mathbf{E}_{t 0}-\mathbf{E}_{h t 0}, 0\right)$ in one case and again (5.13) with $\mathbf{w}-\mathbf{w}_{h}$ replaced by $\left(\mathbf{0}, E_{z}-E_{h z}\right)$ one can proceed as in Section 4.3. In particular, we know that the considered subsequence is always larger than $\epsilon$ and we can achieve the results corresponding to inequality (4.55) and then to inequality (4.56) with $u_{n z}, \mathbf{u}_{n}, \mathbf{u}_{n t 0}$ and $\mathbf{u}_{n t 1}$ replaced respectively by $E_{z}-E_{h z}, \mathbf{E}-\mathbf{E}_{h}, \mathbf{E}_{t 0}-\mathbf{E}_{h t 0}$ and $\mathbf{E}_{t 1}-\mathbf{E}_{h t 1}$. The latter and the previously deduced inequalities give a result which is at odds with (5.13) under the assumed hypotheses, showing convergence of the Galerkin approximation.

Thus, in particular, we can state

Theorem 5.3. Under the hypotheses HD1-HD3, HV1-HV4, HM1-HM6, HM8, HM9, HB1-HB2, HB3S, HMV1HMV5, HSAS1-HSAS3, HSDS2-HSDS5 the sequence $\left\{\mathbf{E}_{h}\right\}_{h \in I_{1}}$ of solutions of Problem 4 strongly converges to $\mathbf{E}$ in $U$, being $\mathbf{E}$ the unique solution of Problem 2.

As we did in [14] it is worth spending a few words about the approximability of Problem 1. In particular, if $\mathbf{B}_{h}, \mathbf{H}_{h}$ and $\mathbf{D}_{h}$ are obtained as functions of $\mathbf{E}_{h}$ and $\mathbf{J}_{m h}$ through formulas that exactly parallel (3.1)-(3.5) and (2.2), then we get $\mathbf{B}_{h} \rightarrow \mathbf{B}, \mathbf{H}_{h} \rightarrow \mathbf{H}$ and $\mathbf{D}_{h} \rightarrow \mathbf{D}$ strongly in $\left(L^{2}(\Omega)\right)^{3}$ as $h \rightarrow 0$. Notice that, even if $\mathbf{H} \in U$, in general, $\mathbf{H}_{h}$ does not converge to $\mathbf{H}$ in $U$ because, in general, $H_{h z} \notin H^{1}(\Omega)$ (see Eq. (3.3)) and $\mathbf{H}_{h t} \notin U_{2 D}$ (see Eq. (3.4)). Hence, the convergence of $\left(\mathbf{E}_{h}, \mathbf{B}_{h}, \mathbf{H}_{h}, \mathbf{D}_{h}\right)$ to the solution $(\mathbf{E}, \mathbf{B}, \mathbf{H}, \mathbf{D})$ of Problem 1 takes place in $U \times\left(L^{2}(\Omega)\right)^{3} \times\left(L^{2}(\Omega)\right)^{3} \times\left(L^{2}(\Omega)\right)^{3}$, even if $(\mathbf{E}, \mathbf{B}, \mathbf{H}, \mathbf{D}) \in U \times\left(L^{2}(\Omega)\right)^{3} \times U \times\left(L^{2}(\Omega)\right)^{3}$ and Problem 1 is well posed in this space.

\section{Finite ElEMENT APPRoximation}

Very often in practice the sequence of finite dimensional subspaces considered in Galerkin method is built by using the finite element method [46]. This is done, as usual, by considering a sequence of triangulations $\left\{\mathcal{T}_{h}\right\}, h \in I$, of $\bar{\Omega}\left([46]\right.$, p. 59) and a specific finite element on each triangulation $\mathcal{T}_{h}$ [46].

In order to avoid the many technicalities involved when curved boundaries are considered ([46], Chap. VI) we assume that ([46], p. 65)

HD4. $\Omega$ is a polygon (i.e., $\left.\bar{\Omega}=\bigcup_{T \in \mathcal{T}_{h}} T\right)$.

For finite element approximations it is usual to make the following basic assumption ([46], p. 131) meaning that no element of the triangulation degenerates as $h \rightarrow 0$

HFE1. the family $\left\{\mathcal{T}_{h}\right\}$ of triangulations is regular.

Finally, the computational electromagnetics community is well aware that Lagrangian and edge elements defined on triangles $[18,34]$ are often the best choices to approximate, respectively, scalar fields belonging to $H^{1}(\Omega)$ and vector fields in any subspace of $H\left(\operatorname{curl}_{2 D}, \Omega\right)$. For these reasons we assume

HFE2. $\mathcal{T}_{h}$ is made up of triangles $\forall h \in I$,

HFE3. edge elements of a given order defined on triangles are used to build $U_{2 D h}, \forall h \in I$.

HFE4. Lagrangian elements of a given order defined on triangles are used to build $U_{h z}, \forall h \in I$.

Now by using classical finite element results we conclude that 
Theorem 6.1. Whenever HD1, HD2, HD4, HFE1, HFE2, HFE3 and HFE4 are satisfied, the space sequence $\left\{U_{h}\right\}$ satisfies condition HSAS1 and HSAS3.

Condition HSAS2 requires additional considerations. In particular, HSAS2 is known to be satisfied for edge elements whenever $\varepsilon_{r}=\mu_{r}=1$ everywhere in $\Omega$ ([18], p. 180). However, it is possible to prove that it holds true for all problems of interest provided that condition HMV3 is satisfied. This is an almost trivial consequence of Proposition 2.27 of ([45], see also pp. 184-185 of [18] taking account that $\varepsilon_{r}$ in those pages should be replaced by $\left.\frac{1+\varepsilon_{r} \mu_{r}-\zeta_{1}}{\mu_{r}}=\frac{\mu_{r} \varepsilon_{r}-\beta^{2}}{\mu_{r}\left(1-\beta^{2}\right)}\right)$.

Taking account of these results, by using Theorem 5.3, we draw the following conclusion for the finite element approximations considered.

Theorem 6.2. Whenever hypotheses HD1-HD4, HV1-HV4, HM1-HM6, HM8, HM9, HB1-HB2, HB3S, HMV1HMV5, HSDS2-HSDS5, HFE1-HFE4 are satisfied, Problem 4 is a convergent approximation of Problem 2.

Remark 6.3. In the above statement we refer to the classical h-version of the finite element method. However, the same conclusions can be achieved also for the p- and hp-version [50] of the finite element method. The interested reader should refer for example to $[48,51]$.

\section{Practical implications}

In this section we would like to show that the developed theory can be used to prove, for the first time to the best of authors's knowledge, the well posedness and finite element approximability of two-dimensional timeharmonic electromagnetic boundary value problems involving non-conducting moving objects with stationary boundaries. The considerations that follow could be useful to show, on the one hand, how such a theory can be exploited. In particular, how one can check the validity of the crucial yet complex conditions HMV2 and HMV5 in cases of practical interest. On the other hand, the same considerations prove that our theory cover broad classes of problems, requiring very weak limitations on the magnitude of the axial velocities of the media involved.

In order to achieve such an outcome we need to deduce some upper or lower bounds for the quantities involved in the indicated conditions. These bounds are obtained by the following lemmas for problems characterized by $\mu_{r}=1$ and $\operatorname{Re}\left(\varepsilon_{r}\right) \geq 1$ in $\Omega_{i}, \forall i \in M$. The same results can be achieved in general, with simple even though much longer calculations.

We start from lemmas which could be of help in understanding when HMV5 holds true.

Lemma 7.1. Let $\varepsilon_{r} \geq 1$ in $\Omega_{i}, i \in M_{\beta}$. Then, under hypotheses HV2, HM3 and HV4 an upper bound for $\left.\frac{|\beta|\left(\varepsilon_{r}-1\right)}{1-\beta^{2}}\right|_{\Omega_{i}}, i \in M_{\beta}$, is given by $\frac{\max _{\mathbf{x} \in \bar{\Omega}_{i}}|\beta|}{1-\max _{\mathbf{x} \in \bar{\Omega}_{i}} \beta^{2}}\left(-1+\max _{\mathbf{x} \in \bar{\Omega}_{i}} \varepsilon_{r}\right)$.

Proof. Let us define the function of two real variables

$$
f:[0,1) \times[1,+\infty) \ni(x, y) \mapsto \frac{x(y-1)}{1-x^{2}} \in \mathbb{R} .
$$

We deduce that $\frac{\partial f}{\partial x}=\frac{\left(1+x^{2}\right)(y-1)}{\left(1-x^{2}\right)^{2}}$ and $\frac{\partial f}{\partial y}=\frac{x}{1-x^{2}}$. Then, in the indicated domain $f$ is not decreasing as a function of $x$ and $y$.

By HV2 (HM3) $\beta\left(\varepsilon_{r}\right)$ is a real-valued function in $\Omega_{i}, \forall i \in M_{\beta}$. Moreover, $0 \leq|\beta| \leq C_{\beta M}<1$ by HV4 and we have assumed $\varepsilon_{r} \geq 1$ in $\Omega_{i}, \forall i \in M_{\beta}$. Thus, we can replace $x$ with $|\beta|, y$ with $\varepsilon_{r}$ and $\beta^{2}$ with $x^{2}$. Moreover, by HV2 (HM3) we know that $\beta\left(\varepsilon_{r}\right)$ has a maximum in $\bar{\Omega}_{i}, \forall i \in M_{\beta}$, so that

$$
\left.\frac{|\beta|\left(\varepsilon_{r}-1\right)}{1-\beta^{2}}\right|_{\Omega_{i}}=f\left(|\beta|, \varepsilon_{r}\right) \leq \frac{\max _{\mathbf{x} \in \bar{\Omega}_{i}}|\beta|}{1-\max _{\mathbf{x} \in \bar{\Omega}_{i}} \beta^{2}}\left(-1+\max _{\mathbf{x} \in \bar{\Omega}_{i}} \varepsilon_{r}\right), \quad i \in M_{\beta} .
$$


Then, for all problems characterized by $\mu_{r}=1$ and $\operatorname{Re}\left(\varepsilon_{r}\right) \geq 1$ in $\Omega_{i}, \forall i \in M$, we can immediately define (see (4.32), (2.6) and HV4)

$$
C_{g l 2}=\frac{C_{\beta M}}{1-C_{\beta M}^{2}}\left(-1+\max _{i \in M_{\beta}}\left(\max _{\mathbf{x} \in \bar{\Omega}_{i}} \varepsilon_{r}\right)\right)
$$

since $\left.\beta\right|_{\Omega_{k}}=0$ and, then, $\left.\zeta_{2}\right|_{\Omega_{k}}=0 \forall k \in M_{0}$.

The proofs of the next two lemmas are very similar to the one of Lemma 3 and, for this reason, they are omitted.

Lemma 7.2. Let $\varepsilon_{r} \geq 1$ in $\Omega_{i}, i \in M_{\beta}$. Then, under hypotheses HV2, HM3 and HV4 a lower bound for $\left.\frac{\varepsilon_{r}-\beta^{2}}{1-\beta^{2}}\right|_{\Omega_{i}}, i \in M_{\beta}$, is given by $\left(\frac{\min _{\mathbf{x} \in \bar{\Omega}_{i}} \varepsilon_{r}-\min _{\mathbf{x} \in \bar{\Omega}_{i}} \beta^{2}}{1-\min _{\mathbf{x} \in \bar{\Omega}_{i}} \beta^{2}}\right) \geq \min _{\mathbf{x} \in \bar{\Omega}_{i}} \varepsilon_{r}$.

In this case, taking account of the definition of $C_{m v 3}$ in hypothesis HMV3 and of the fact that the moving media are characterized by real-valued $\varepsilon_{r}$, we set

$$
C_{m v 3}=\min _{i \in M}\left(\min _{\mathbf{x} \in \bar{\Omega}_{i}} \operatorname{Re}\left(\varepsilon_{r}\right)\right)
$$

again for problems characterized by $\mu_{r}=1$ and $\operatorname{Re}\left(\varepsilon_{r}\right) \geq 1$ in $\Omega_{i}, \forall i \in M$.

Lemma 7.3. Let $\varepsilon_{r} \geq 1$ in $\Omega_{i}, i \in M_{\beta}$. Then, under hypotheses HV2, HM3 and HV4 a lower bound for $\left.\frac{1-\varepsilon_{r} \beta^{2}}{1-\beta^{2}}\right|_{\Omega_{i}}, i \in M_{\beta}$, is given by $\frac{1-\left(\max _{\mathbf{x} \in \bar{\Omega}_{i}} \beta^{2}\right) \max _{\mathbf{x} \in \bar{\Omega}_{i}} \varepsilon_{r}}{1-\max _{\mathbf{x} \in \bar{\Omega}_{i}} \beta^{2}} \leq 1$.

By using Lemma 7.3, whenever $\mu_{r}=1$ and $\varepsilon_{r} \geq 1$ in $\Omega_{i}, \forall i \in M_{\beta}$, and

$$
C_{\beta M}^{2} \max _{i \in M_{\beta}}\left(\max _{\mathbf{x} \in \bar{\Omega}_{i}} \varepsilon_{r}\right)<1
$$

we define (see hypothesis HMV4)

$$
0<C_{m v 4}=\frac{1-C_{\beta M}^{2} \max _{i \in M_{\beta}}\left(\max _{\mathbf{x} \in \bar{\Omega}_{i}} \varepsilon_{r}\right)}{1-C_{\beta M}^{2}} \leq 1 .
$$

Moreover, whenever $\mu_{r}=1$ in $\Omega_{i}, \forall i \in M_{0}$, we can choose $C_{\mu M k}=1 \forall k \in M_{0}$ and, by using (4.31), we define

$$
C_{m v 4 m}=C_{m v 4} \text {. }
$$

We are now ready to deduce one of the most important lemmas of this section.

Lemma 7.4. Let us assume that the hypotheses we have considered in the definitions of Problems 1 and 2 are satisfied. Then, if $\mu_{r}=1$ and $\operatorname{Re}\left(\varepsilon_{r}\right) \geq 1$ in $\Omega_{i}, \forall i \in M$, condition HMV5 is satisfied if the following inequality, involving just $\max _{i \in M_{\beta}}\left(\max _{\mathbf{x} \in \bar{\Omega}_{i}} \varepsilon_{r}\right), \min _{i \in M}\left(\min _{\mathbf{x} \in \bar{\Omega}_{i}} \operatorname{Re}\left(\varepsilon_{r}\right)\right)$ and $C_{\beta M}$, holds true

$$
\frac{C_{\beta M}^{2}\left(-1+\max _{i \in M_{\beta}}\left(\max _{\mathbf{x} \in \bar{\Omega}_{i}} \varepsilon_{r}\right)\right)^{2}}{\left(1-C_{\beta M}^{2}\right)\left(1-C_{\beta M}^{2} \max _{i \in M_{\beta}}\left(\max _{\mathbf{x} \in \bar{\Omega}_{i}} \varepsilon_{r}\right)\right) \min _{i \in M}\left(\min _{\mathbf{x} \in \bar{\Omega}_{i}} \operatorname{Re}\left(\varepsilon_{r}\right)\right)}<1,
$$

provided that $C_{\beta M}$ and $\varepsilon_{r}$ satisfy (7.5).

Proof. Our assumptions allows us to exploit the previous results. Then, (7.8) is a direct consequence of the definition of HMV5, where $C_{g l 2}, C_{m v 3}$ and $C_{m v 4}$ are replaced by the quantities provided by (7.3), (7.4) and (7.7) (see also (7.6)), respectively. 
Now we focus on lemmas which will be useful to show that HMV2 holds true. Their proofs are not reported since they are very similar to the one of Lemma 3.

Lemma 7.5. Let $\varepsilon_{r} \geq 1$ in $\Omega_{i}, i \in M_{\beta}$. Then, under hypotheses HV2, HM3 and HV4 a lower bound for $\left.\frac{\left(1-\beta^{2}\right)^{2}}{\varepsilon_{r}\left(\varepsilon_{r}-\beta^{2}\right)^{2}}\right|_{\Omega_{i}}, i \in M_{\beta}$, is given by $\frac{\left(1-\max _{\mathbf{x} \in \overline{\Omega_{i}}} \beta^{2}\right)^{2}}{\left(\max _{\mathbf{x} \in \bar{\Omega}_{i}} \varepsilon_{r}\right)\left(\max _{\mathbf{x} \in \bar{\Omega}_{i}} \beta^{2}-\max _{\mathbf{x} \in \bar{\Omega}_{i}} \varepsilon_{r}\right)^{2}}$.

Then, for all problems characterized by $\mu_{r}=1$ and $\varepsilon_{r} \geq 1$ in $\Omega_{i}, \forall i \in M_{\beta}$, we immediately deduce (see (4.4))

$$
C_{k, d, i} \geq \frac{\left(1-\max _{\mathbf{x} \in \bar{\Omega}_{i}} \beta^{2}\right)^{2}}{\varepsilon_{0}^{3}\left(\max _{\mathbf{x} \in \bar{\Omega}_{i}} \varepsilon_{r}\right)\left(\max _{\mathbf{x} \in \bar{\Omega}_{i}} \beta^{2}-\max _{\mathbf{x} \in \bar{\Omega}_{i}} \varepsilon_{r}\right)^{2}}, i \in M_{\beta} .
$$

Moreover, under the same conditions (that is $\mu_{r}=1, \varepsilon_{r} \geq 1$ in $\Omega_{i}, \forall i \in M_{\beta}$ ), from Definitions (4.6) and (4.2), taking account of Lemma 7.2, we get

$$
C_{k, r, i} \geq \varepsilon_{0} \min _{\mathbf{x} \in \bar{\Omega}_{i}} \varepsilon_{r}, i \in M_{\beta},
$$

and

$$
C_{k, s, i} \leq \frac{1}{\varepsilon_{0}}\left(\frac{1-\min _{\mathbf{x} \in \bar{\Omega}_{i}} \beta^{2}}{\min _{\mathbf{x} \in \bar{\Omega}_{i}} \varepsilon_{r}-\min _{\mathbf{x} \in \bar{\Omega}_{i}} \beta^{2}}+\frac{1}{\min _{\mathbf{x} \in \bar{\Omega}_{i}} \varepsilon_{r}}\right), i \in M_{\beta} .
$$

We now deduce the corresponding results for $C_{\nu, d, i}, C_{\nu, r, i}$ and $C_{\nu, s, i}$.

Lemma 7.6. Let $\varepsilon_{r} \geq 1$ in $\Omega_{i}, i \in M_{\beta}$. Then, under hypotheses HV2, HM3 and HV4 a lower bound for

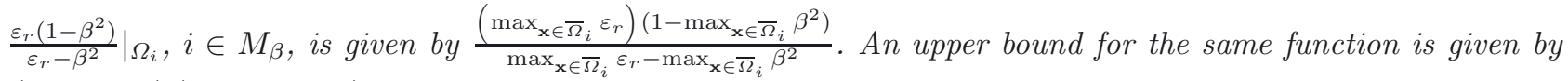
$\frac{\left(\min _{\mathbf{x} \in \bar{\Omega}_{i}} \varepsilon_{r}\right)\left(1-\min _{\mathbf{x} \in \bar{\Omega}_{i}} \beta^{2}\right)}{\min _{\mathbf{x} \in \bar{\Omega}_{i}} \varepsilon_{r}-\min _{\mathbf{x} \in \bar{\Omega}_{i}} \beta^{2}} \leq 1$.

Then, in analogy to what we have seen before, for all problems characterized by $\mu_{r}=1$ and $\varepsilon_{r} \geq 1$ in $\Omega_{i}$, $\forall i \in M_{\beta}$, we get (see (4.5))

$$
C_{\nu, d, i} \geq \frac{\left(\max _{\mathbf{x} \in \bar{\Omega}_{i}} \varepsilon_{r}\right)^{2}\left(1-\max _{\mathbf{x} \in \bar{\Omega}_{i}} \beta^{2}\right)^{2}}{\mu_{0}^{3}\left(\max _{\mathbf{x} \in \bar{\Omega}_{i}} \beta^{2}-\max _{\mathbf{x} \in \bar{\Omega}_{i}} \varepsilon_{r}\right)^{2}}, i \in M_{\beta} .
$$

As before, under the same conditions (that is $\mu_{r}=1, \varepsilon_{r} \geq 1$ in $\Omega_{i}, \forall i \in M_{\beta}$ ), from Definitions (4.7) and (4.3), taking account of Lemma 7.6, we get

$$
C_{\nu, r, i} \geq \mu_{0}, i \in M_{\beta},
$$

and

$$
C_{\nu, s, i} \leq \frac{1}{\mu_{0}}\left(1+\frac{\left(\min _{\mathbf{x} \in \bar{\Omega}_{i}} \varepsilon_{r}\right)\left(1-\min _{\mathbf{x} \in \bar{\Omega}_{i}} \beta^{2}\right)}{\min _{\mathbf{x} \in \bar{\Omega}_{i}} \varepsilon_{r}-\min _{\mathbf{x} \in \bar{\Omega}_{i}} \beta^{2}}\right) \leq \frac{2}{\mu_{0}}, i \in M_{\beta} .
$$

Finally, for the left-hand side of inequality (4.1) we have:

Lemma 7.7. Let $\varepsilon_{r} \geq 1$ in $\Omega_{i}, i \in M_{\beta}$. Then, under hypotheses HV2, HM3 and HV4 an upper bound for $\left.\frac{|\beta|\left(\varepsilon_{r}-1\right)}{\varepsilon_{r}-\beta^{2}}\right|_{\Omega_{i}}, i \in M_{\beta}$, is given by $\frac{\left(\max _{\mathbf{x} \in \bar{\Omega}_{i}}|\beta|\right)\left(1-\max _{\mathbf{x} \in \bar{\Omega}_{i}} \varepsilon_{r}\right)}{\max _{\mathbf{x} \in \bar{\Omega}_{i}} \beta^{2}-\max _{\mathbf{x} \in \bar{\Omega}_{i}} \varepsilon_{r}}$.

Thus, for all problems characterized by $\mu_{r}=1$ and $\varepsilon_{r} \geq 1$ in $\Omega_{i}, \forall i \in M_{\beta}$, we deduce

$$
\max _{\mathbf{x} \in \bar{\Omega}_{i}}\left(\frac{\beta^{2}\left(\mu_{r} \varepsilon_{r}-1\right)^{2}}{\left(\mu_{r} \varepsilon_{r}-\beta^{2}\right)^{2}}\right) \leq \frac{\left(\max _{\mathbf{x} \in \bar{\Omega}_{i}} \beta^{2}\right)\left(1-\max _{\mathbf{x} \in \bar{\Omega}_{i}} \varepsilon_{r}\right)^{2}}{\left(\max _{\mathbf{x} \in \bar{\Omega}_{i}} \beta^{2}-\max _{\mathbf{x} \in \bar{\Omega}_{i}} \varepsilon_{r}\right)^{2}}, i \in M_{\beta} .
$$

With these results we can easily obtain 
Lemma 7.8. Let us assume that the hypotheses we have considered in the definitions of Problems 1 and 2 are satisfied. Then, if $\mu_{r}=1$ and $\varepsilon_{r} \geq 1$ in $\Omega_{i}, \forall i \in M_{\beta}$, condition HMV2 is satisfied if the following inequality holds true for all $i \in M_{\beta}$

$$
\begin{aligned}
& \frac{16\left(\max _{\mathbf{x} \in \bar{\Omega}_{i}} \beta^{2}\right)\left(1-\max _{\mathbf{x} \in \bar{\Omega}_{i}} \varepsilon_{r}\right)^{2}}{\left(\max _{\mathbf{x} \in \bar{\Omega}_{i}} \beta^{2}-\max _{\mathbf{x} \in \bar{\Omega}_{i}} \varepsilon_{r}\right)^{2}} \\
& <\left(-\frac{1-\min _{\mathbf{x} \in \bar{\Omega}_{i}} \beta^{2}}{\min _{\mathbf{x} \in \bar{\Omega}_{i}} \varepsilon_{r}-\min _{\mathbf{x} \in \bar{\Omega}_{i}} \beta^{2}}-\frac{1}{\min _{\mathbf{x} \in \bar{\Omega}_{i}} \varepsilon_{r}}\right. \\
& +\sqrt{\left(\frac{1-\min _{\mathbf{x} \in \bar{\Omega}_{i}} \beta^{2}}{\min _{\mathbf{x} \in \bar{\Omega}_{i}} \varepsilon_{r}-\min _{\mathbf{x} \in \bar{\Omega}_{i}} \beta^{2}}+\frac{1}{\min _{\mathbf{x} \in \bar{\Omega}_{i}} \varepsilon_{r}}\right)^{2}+\frac{4\left(1-\max _{\mathbf{x} \in \bar{\Omega}_{i}} \beta^{2}\right)^{2} \min _{\mathbf{x} \in \bar{\Omega}_{i}} \varepsilon_{r}}{\left(\max _{\mathbf{x} \in \bar{\Omega}_{i}} \varepsilon_{r}\right)\left(\max _{\mathbf{x} \in \bar{\Omega}_{i}} \beta^{2}-\max _{\mathbf{x} \in \bar{\Omega}_{i}} \varepsilon_{r}\right)^{2}}} \\
& \times\left(-1-\frac{\left(\min _{\mathbf{x} \in \bar{\Omega}_{i}} \varepsilon_{r}\right)\left(1-\min _{\mathbf{x} \in \bar{\Omega}_{i}} \beta^{2}\right)}{\min _{\mathbf{x} \in \bar{\Omega}_{i}} \varepsilon_{r}-\min _{\mathbf{x} \in \bar{\Omega}_{i}} \beta^{2}}\right. \\
& +\sqrt{\left(1+\frac{\left(\min _{\mathbf{x} \in \bar{\Omega}_{i}} \varepsilon_{r}\right)\left(1-\min _{\mathbf{x} \in \bar{\Omega}_{i}} \beta^{2}\right)}{\min _{\mathbf{x} \in \bar{\Omega}_{i}} \varepsilon_{r}-\min _{\mathbf{x} \in \bar{\Omega}_{i}} \beta^{2}}\right)^{2}+\frac{4\left(1-\max _{\mathbf{x} \in \bar{\Omega}_{i}} \beta^{2}\right)^{2}\left(\max _{\mathbf{x} \in \bar{\Omega}_{i}} \varepsilon_{r}\right)^{2}}{\left(\max _{\mathbf{x} \in \bar{\Omega}_{i}} \beta^{2}-\max _{\mathbf{x} \in \bar{\Omega}_{i}} \varepsilon_{r}\right)^{2}}} .
\end{aligned}
$$

Proof. Our assumptions allows us to exploit the previous results. Moreover, if we define the function of three real variables

$$
f:(0,+\infty)^{3} \ni(x, y, z) \mapsto-x+\sqrt{x^{2}+4 y z} \in \mathbb{R}
$$

we can easily deduce that $\frac{\partial f}{\partial x}=-1+\frac{x}{\sqrt{x^{2}+4 y z}}, \frac{\partial f}{\partial y}=\frac{2 z}{\sqrt{x^{2}+4 y z}}$ and $\frac{\partial f}{\partial z}=\frac{2 y}{\sqrt{x^{2}+4 y z}}$. Therefore, in the indicated domain $f$ is increasing as a function of the variable $y$ and $z$ and decreasing as a function of the variable $x$. From this property, taking account of (7.9)-(7.14), we get that for any $i \in M_{\beta}$ the right-hand side of equation (7.16) is smaller than the right-hand side of (4.1). Moreover, by using (7.15), the left-hand side of equation (7.16) is larger than or equal to the left-hand side of (4.1). Thus, if condition (7.16) is satisfied for all $i \in M_{\beta}$, the validity of HMV2 is guaranteed.

Now we observe that, whenever $\mu_{r}=1$ and $\operatorname{Re}\left(\varepsilon_{r}\right) \geq 1$ in $\Omega_{i}, \forall i \in M$, and (7.5) holds true, conditions HMV3 and HMV4 are satisfied (see, respectively, (7.4) and (7.6)). Moreover, $\mu_{r}=1$ and $\operatorname{Re}\left(\varepsilon_{r}\right) \geq 1$ in $\Omega_{i}, \forall i \in M$, together with HV4, imply that HMV1 holds true as well.

With these results, by recalling Theorems 4.17 and 6.2 , it is now trivial to deduce

Corollary 7.9. Under the hypotheses HD1-HD3, HV1-HV4, HM1-HM6, HM8, HM9, HB1-HB2 and HB3S, Problem 2 is well posed whenever $\mu_{r}=1$ and $\operatorname{Re}\left(\varepsilon_{r}\right) \geq 1$ in $\Omega_{i}, \forall i \in M$, and $\varepsilon_{r}$ and $\beta$ satisfy (7.5), (7.8) and (7.16).

Corollary 7.10. Under the hypotheses HD1-HD4, HV1-HV4, HM1-HM6, HM8, HM9, HB1-HB2, HB3S, HSDS2-HSDS5 and HFE1-HFE4 Problem 4 is a convergent approximation of Problem 2 whenever $\mu_{r}=1$ and $\operatorname{Re}\left(\varepsilon_{r}\right) \geq 1$ in $\Omega_{i}, \forall i \in M$, and $\varepsilon_{r}$ and $\beta$ satisfy (7.5), (7.8) and (7.16).

The three conditions (7.5), (7.8) and (7.16) are still rather complex and abstract. We now focus on more practical applications and propose a simple approach to manage them, which allows to deduce very interesting results. In particular, for any specific problem of interest and any fixed value of $\mathrm{n}, n \in \mathbb{N}, n \geq 1$, by using Remark 2.2, we introduce a possibly finer decomposition of $\Omega$ satisfying HD3 and such that

$$
\max _{i \in M_{\beta}}\left(\max _{\mathbf{x} \in \bar{\Omega}_{i}} \varepsilon_{r}-\min _{\mathbf{x} \in \bar{\Omega}_{i}} \varepsilon_{r}\right) \leq \frac{1}{n} .
$$


Our aim is to find a set of values, $C_{\beta M i}=\max _{\mathbf{x} \in \bar{\Omega}_{i}}|\beta|\left(\leq C_{\beta M}<1\right.$ by HV4) $i \in M_{\beta}$, allowing to apply our theory whenever $0 \leq|\beta|_{\Omega_{i}} \mid \leq C_{\beta M i} \forall i \in M_{\beta}$.

To fix the ideas, we assume that $\min _{i \in M_{0}}\left(\min _{\mathbf{x} \in \bar{\Omega}_{i}} \operatorname{Re}\left(\varepsilon_{r}\right)\right)=1$ (having in mind scattering problems in free space), in addition to $\mu_{r}=1$ and $\operatorname{Re}\left(\varepsilon_{r}\right) \geq 1$ in $\Omega_{i}, \forall i \in M$.

As the reader can easily check, the bounds provided by the global inequalities (7.5) and (7.8) determines the upper bound $C_{\beta M}$ in terms of the maximum of the relative permittivity in the moving media, when $\min _{i \in M_{0}}\left(\min _{\mathbf{x} \in \bar{\Omega}_{i}} \operatorname{Re}\left(\varepsilon_{r}\right)\right)=1$. This is completely independent of the possibly finer decomposition of $\Omega$ introduced above since, by definition, any additional splitting of $\Omega_{i}$ for $i \in M_{\beta}$ is not able to change $\max _{i \in M_{\beta}}\left(\max _{\mathbf{x} \in \bar{\Omega}_{i}} \varepsilon_{r}\right)$.

On the contrary, inequality (7.16) provides a bound for any specific $i \in M_{\beta}$. Since we assume no restriction on $\min _{\mathbf{x} \in \bar{\Omega}_{i}} \beta^{2}$ in order to carry out a worst-case analysis we set this quantity to zero, for all $i \in M_{\beta}$. Then, if we substitute $C_{\varepsilon M i}$ for $\max _{\mathbf{x} \in \bar{\Omega}_{i}} \varepsilon_{r}, i \in M_{\beta}$, inequality (7.16) reduces to

$$
\begin{aligned}
\frac{4 C_{\beta M i}^{2}\left(1-C_{\varepsilon M i}\right)^{2}}{\left(C_{\beta M i}^{2}-C_{\varepsilon M i}\right)^{2}} & \left.<-\frac{1}{\min _{\mathbf{x} \in \bar{\Omega}_{i}} \varepsilon_{r}}+\sqrt{\frac{1}{\left(\min _{\mathbf{x} \in \bar{\Omega}_{i}} \varepsilon_{r}\right)^{2}}+\frac{\left(1-C_{\beta M i}^{2}\right)^{2} \min _{\mathbf{x} \in \bar{\Omega}_{i} \varepsilon_{r}}}{C_{\varepsilon M i}\left(C_{\beta M i}^{2}-C_{\varepsilon M i}\right)^{2}}}\right) \\
& \times\left(-1+\sqrt{1+\frac{\left(1-C_{\beta M i}^{2}\right)^{2} C_{\varepsilon M i}^{2}}{\left(C_{\beta M i}^{2}-C_{\varepsilon M i}\right)^{2}}}\right), \quad i \in M_{\beta} .
\end{aligned}
$$

Moreover, taking account of (7.18), we get $\min _{\mathbf{x} \in \bar{\Omega}_{i}} \varepsilon_{r} \geq C_{\varepsilon M i}-\frac{1}{n}$. Thus, by using again the same arguments adopted in the proof of Lemma 7.8, we perform a worst-case analysis by replacing $\min _{\mathbf{x} \in \bar{\Omega}_{i}} \varepsilon_{r}$ with $C_{\varepsilon M i}-\frac{1}{n}$. After the substitution, the following sufficient condition for the validity of inequality (7.19) is obtained

$$
\begin{aligned}
\frac{4 C_{\beta M i}^{2}\left(1-C_{\varepsilon M i}\right)^{2}}{\left(C_{\beta M i}^{2}-C_{\varepsilon M i}\right)^{2}} & \left.<-\frac{1}{C_{\varepsilon M i}-\frac{1}{n}}+\sqrt{\frac{1}{\left(C_{\varepsilon M i}-\frac{1}{n}\right)^{2}}+\frac{\left(1-C_{\beta M i}^{2}\right)^{2}\left(C_{\varepsilon M i}-\frac{1}{n}\right)}{C_{\varepsilon M i}\left(C_{\beta M i}^{2}-C_{\varepsilon M i}\right)^{2}}}\right) \\
& \times\left(-1+\sqrt{1+\frac{\left(1-C_{\beta M i}^{2}\right)^{2} C_{\varepsilon M i}^{2}}{\left(C_{\beta M i}^{2}-C_{\varepsilon M i}\right)^{2}}}\right), \quad i \in M_{\beta} .
\end{aligned}
$$

The results deduced by using this approach are shown in Figure 1. The two plots deduced from inequalities (7.5) and (7.8) provides $C_{\beta M}$ as a function of $\max _{i \in M_{\beta}} C_{\varepsilon M i}$ (indicated as $\max _{i} C_{\varepsilon M i}$ in Fig. 1), while the three plots obtained by using inequality (7.20) gives $C_{\beta M i}$ versus $C_{\varepsilon M i}$.

As it is trivial to check, we can neglect the plot due to inequality (7.5), since inequality (7.8) provides in any case more restrictive results.

On the contrary, all plots deduced by inequality (7.20) are very similar. The only significant differences can be noticed for small values of $C_{\varepsilon M i}$ between the plot deduced when $n=1$ and the ones obtained when $n=10$ or $n=100$. This is related to the fact that when $C_{\varepsilon M i}$ is small $n=1$ allows the moving media to be significantly inhomogeneous (i.e., $C_{\varepsilon M i}=2$ and $n=1$ means that $\min _{\mathbf{x} \in \bar{\Omega}_{i}} \varepsilon_{r}$ could be equal to 1 ) while this is not possible when $n=10$ or $n=100$. Moreover, for $n=1$ the plot starts from $C_{\varepsilon M i} \geq 2$, in order to satisfy the hypothesis $\operatorname{Re}\left(\varepsilon_{r}\right) \geq 1$ in $\Omega_{i}$. However, it is important to notice that, on the one hand, for any problem of interest we can always set $n=10$ or $n=100$ and that, on the other hand, it is almost useless to consider larger values of $n$. Thus, of the three plots of Figure 1 related to inequality (7.20) we can focus just on the one obtained for $n=100$. 


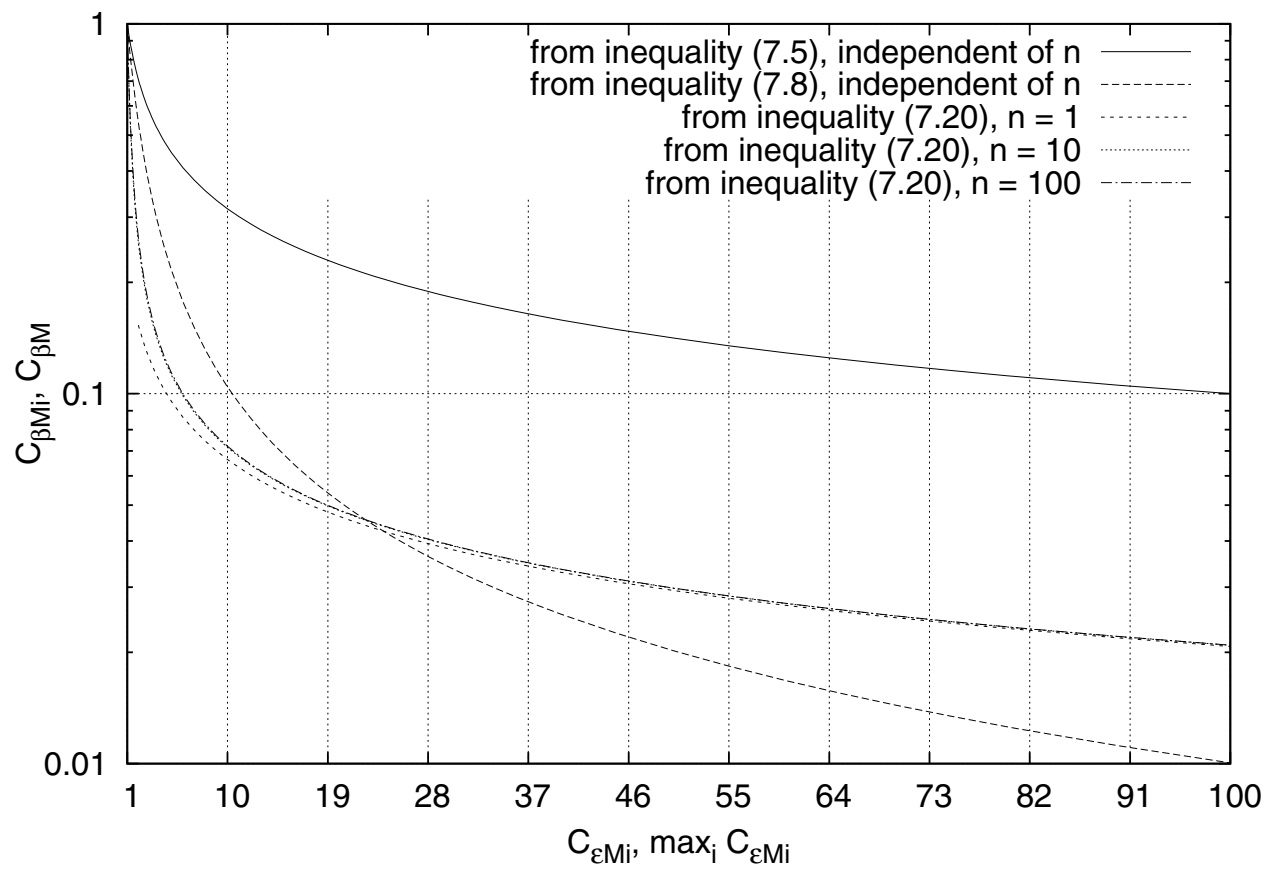

FiguRE 1. Different upper bounds for $|\beta|$ required by inequalities (7.5), (7.8) and (7.20). Inequalities (7.5) and (7.8) provide $C_{\beta M}$ versus $\max _{i \in M_{\beta}} C_{\varepsilon M i}$, while inequality (7.20) gives $C_{\beta M i}$ versus $C_{\varepsilon M i}$ for different values of $n$.

For these reasons, our next comments will be related just to the two plots deduced from inequality (7.20) for $n=100$ and inequality (7.8).

The first trivial comment we deduce from Figure 1 is that for almost all non-conducting media we can find in nature (satisfying the hypotheses considered in this section, that is $\mu_{r}=1$ and $\operatorname{Re}\left(\varepsilon_{r}\right) \geq 1$ in $\Omega_{i}$, $\forall i \in M)$ we can apply our theory whenever $|\beta| \leq 0.01$, that is to say when the axial velocity is not larger than $0.01 c_{0} \simeq 3000 \mathrm{~km} / \mathrm{s}$, since $\varepsilon_{r} \leq 100$ is not at all restrictive. This speed limit is already so large to cover most problems of practical interest without any additional consideration.

In order to find sharper results it is crucial to observe that the lines deduced from inequalities (7.8) and (7.20) for $n=100$ meet when $C_{\varepsilon M i}=\max _{i \in M_{\beta}} C_{\varepsilon M i} \simeq 22.36$.

For problems involving media having $\max _{i \in M_{\beta}} C_{\varepsilon M i} \geq 22.36$ we deduce one global upper bound for the magnitude of the axial speed of all moving materials: the one given by inequality (7.8). To give an example, if $\max _{i \in M_{\beta}} C_{\varepsilon M i}=46$ we deduce $C_{\beta M} \simeq 0.022$ and $C_{\beta M i}=C_{\beta M}$ for all $i \in M_{\beta}$ even though in a subdomain $\Omega_{j}$, $j \in M_{\beta}$, we have $C_{\varepsilon M i}=2$.

On the contrary, if $\max _{i \in M_{\beta}} C_{\varepsilon M i}<22.36$ we can deduce different values for $C_{\beta M i}, i \in M_{\beta}$. In these cases we have a global bound, again given by inequality (7.8), and more specific bounds given by inequality (7.20) for $n=100$. For example, if $\max _{i \in M_{\beta}} C_{\varepsilon M i}=10$ we get $C_{\beta M} \leq 0.104$ (from inequality (7.8)). However, all subdomains $\Omega_{j}, j \in M_{\beta}$, where $\varepsilon_{r}$ reaches its maximum value the right bound for the magnitude of the axial speed is $C_{\beta M j}=0.072$ (from inequality (7.20) for $n=100$ ). If, in the same problem, we have a subdomain $\Omega_{k}$, $k \in M_{\beta}$, where $C_{\varepsilon M k}$ is equal to 6 , then we get $C_{\beta M k}=0.1$ (from (7.20) for $n=100$ ). But in case we had also subdomains $\Omega_{l}, l \in M_{\beta}$, characterized by $C_{\varepsilon M k}=2$ we are not allowed to deduce $C_{\beta M l}=0.26$ (from (7.20) for $n=100$ ) because the upper bound determined by inequality (7.8) gives, as already pointed out, $C_{\beta M i} \leq 0.104$ for all $i \in M_{\beta}$. 


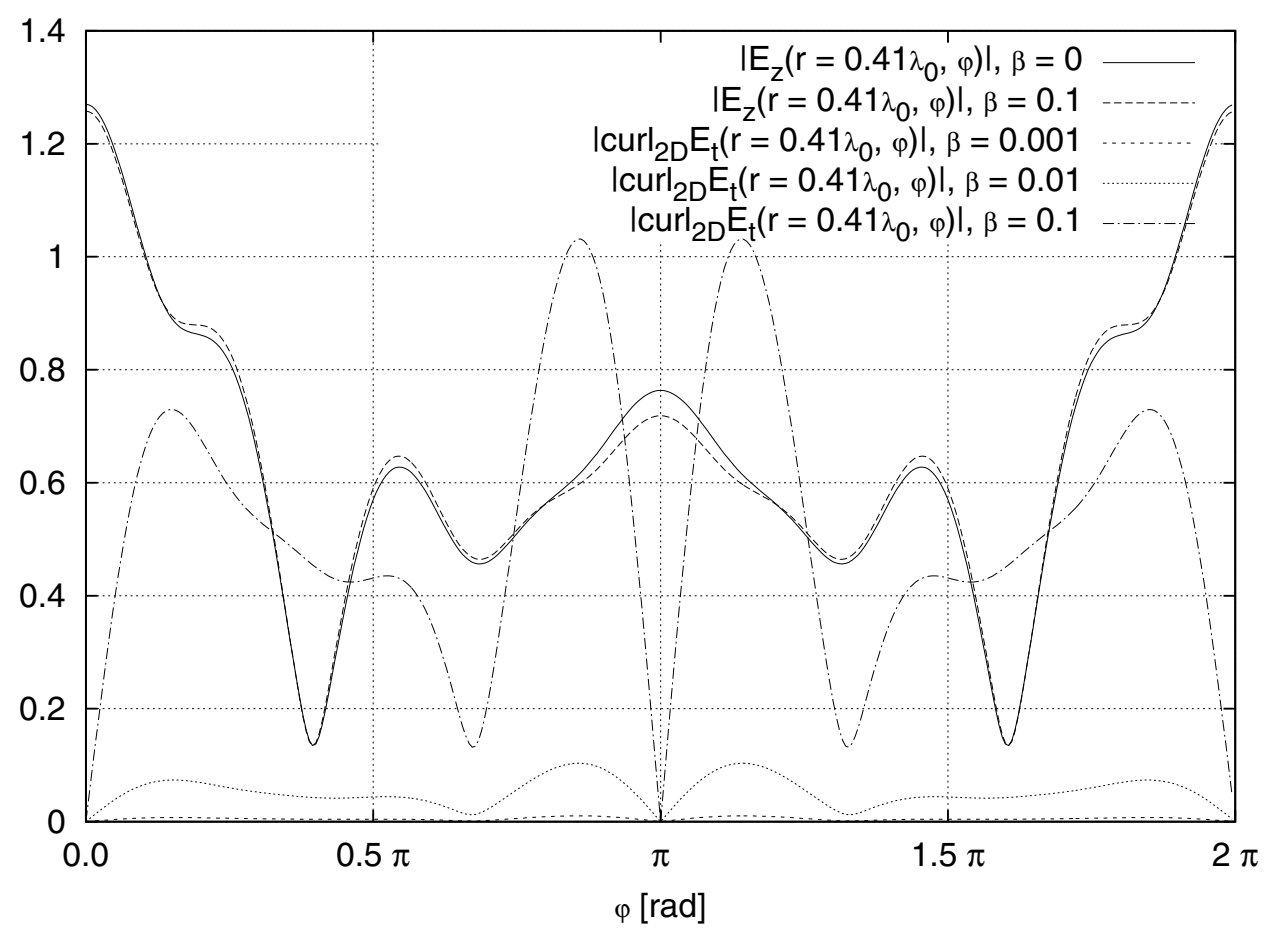

Figure 2. Behaviours of $\left|E_{z}\right|$ and $\left|\operatorname{curl}_{2 D} \mathbf{E}_{t}\right|$ for different values of $\beta$ along the circle of radius $0.41 \lambda_{0} . r$ and $\varphi$ are the usual polar coordinates.

Remark 7.11. So far in this section we have considered $\varepsilon_{r}$ as a quantity defined in advance and have deduced the set of bounds $C_{\beta M i}, i \in M_{\beta}$. However, analogous results can be deduced if one considers $\beta$ as a field defined in advance and look for the set of bounds $C_{\varepsilon M i}, i \in M_{\beta}$.

Finally, in order to conclude our work, we present some additional results for problems involving canonical scatterers in axial movement [11]. These results will be useful, on the one hand, to show the qualitative effects of the presence of axially moving materials and, on the other hand, to point out that in many cases covered by our theory the solutions which can be obtained by neglecting all axial movements cannot be considered as approximations of the solutions of interest, not even as rough ones.

We consider, in particular, an elliptic pipe, that is a two-layer elliptic cylinder hosted in free space. The center of gravity of the cylinder is placed at the origin of the coordinate system and the semimajor axes are assumed to lay on the $x$-axis. The semifocal distance is set to $0.2 \lambda_{0}$ and the semimajor axes are $0.4 \lambda_{0}$ and $0.5 \lambda_{0}$, where $\lambda_{0}$ is the wavelength in vacuum. The constitutive parameters of the involved media are (in their rest frames) $\varepsilon_{r}=5.0, \mu_{r}=1.0, \sigma=0.0$ for the inner layer of the scatterer and $\varepsilon_{r}=2.0, \mu_{r}=1.0, \sigma=0.0$ for the exterior one. In any case the exterior layer of the scatterer is at rest in the selected reference frame (the pipe is at rest) while the interior one can move at different velocities so that $|\beta|$ varies in the range $[0,0.1]$ (the material inside the pipe is in motion). For the indicated parameters our theory applies. As a matter of fact, we can apply the approach presented in this section. Since we are in the presence of piecewise homogeneous materials, no additional subdomains need to be defined, even for $n \rightarrow+\infty$. Therefore, from Figure 1 we get $C_{\beta M}=0.113$. We assume that the scatterer is illuminated by a plane wave having $\mathbf{E}=E_{z} \hat{\mathbf{z}}$, with $E_{z}=e^{-j \frac{2 \pi x}{\lambda_{0}}}$. With this illumination we get $\mathbf{E}_{t}=0$ everywhere whenever all materials are at rest. However, in the presence of an axial movement, this is not true anymore. In particular, in Figure 2 we show the behaviours of $\left|E_{z}\right|$ and $\left|\operatorname{curl}_{2 D} \mathbf{E}_{t}\right|$ along the circle of radius $0.41 \lambda_{0}$ (in the pipe region) for different values of $\beta$. The results clearly show the effect 
of the motion. Such an effect is relatively small for $\left|E_{z}\right|$ and, for this reason, just two plots related to $\left|E_{z}\right|$ are shown in Figure 2: one for $\beta=0$ and the other for $\beta$ equal to the maximum value considered. On the contrary, the effect of $\beta$ on $\left|\operatorname{curl}_{2 D} \mathbf{E}_{t}\right|$ is really impressive. In particular, $\left|\operatorname{curl}_{2 D} \mathbf{E}_{t}\right|$ is equal to zero when all media are motionless, is not negligible for all values of $\beta$ considered in Figure 2, at least in parts of the considered circle, and becomes even larger than $\left|E_{z}\right|$ for $\beta=0.1$, again on a subset of the circle. Now, we easily get the result we looked for. As a matter of fact, $\left|\operatorname{curl}_{2 D} \mathbf{E}_{t}\right|$ determines a part of the $U$ norm of the difference between the solution obtained when the motion is neglected and the one computed by considering it.

\section{Conclusions}

In this work we have dealt with a two-dimensional time-harmonic electromagnetic boundary value problem involving non-conducting moving objects with stationary boundaries. Sufficient conditions for well-posedness and finite element approximability of this problem have been presented. The crucial constraints of this set of sufficient conditions involve just the constitutive parameters of the media and the velocity field. Practical implications of the developed theory have been discussed. It has been shown, in particular, that many problems of practical interest are covered by the proposed theory.

\section{REFERENCES}

[1] J.G. Van Bladel, Electromagnetic Fields, 2nd edition. IEEE Press, Piscataway, NJ, USA (2007).

[2] D.K. Cheng and J.-A. Kong, Covariant descriptions of bianisotropic media. Proc. IEEE 56 (1968) $248-251$.

[3] A.M. Messiaen and P.E. Vandenplas, High-frequency effect due to the axial drift velocity of a plasma column. Phys. Rev. 149 (1966) 131-140.

[4] C. Yeh, Scattering obliquely incident microwaves by a moving plasma column. J. Appl. Phys. 40 (1969) 5066-5075.

[5] T. Shiozawa and S. Seikai, Scattering of electromagnetic waves from an inhomogeneous magnetoplasma column moving in the axial direction. IEEE Trans. Antennas Propag. 20 (1972) 455-463.

[6] J.V. Parker, J.C. Nickel and R.W. Gould, Resonance oscillations in a hot nonuniform plasma. Phys. Fluids 7 (1964) 1489-1500.

[7] D. Censor, Scattering of electromagnetic waves by a cylinder moving along its axis. IEEE Trans. Microwave Theory Tech. 17 (1969) 154-158.

[8] Y. Yan, Mass flow measurement of bulk solids in pneumatic pipelines. Meas. Sci. Technol. 7 (1996) 1687

[9] C. Yeh, Reflection and transmission of electromagnetic waves by a moving dielectric medium. J. Appl. Phys. 36 (1965) 3513-3517.

[10] V.P. Pyati, Reflection and refraction of electromagnetic waves by a moving dielectric medium. J. Appl. Phys. 38 (1967) $652-655$.

[11] M. Pastorino and M. Raffetto, Scattering of electromagnetic waves from a multilayer elliptic cylinder moving in the axial direction. IEEE Trans. Antennas Propag. 61 (2013) 4741-4753.

[12] A. Freni, C. Mias and R.L. Ferrari, Finite element analysis of electromagnetic wave scattering by a cylinder moving along its axis surrounded by a longitudinal corrugated structure. IEEE Trans. Magn. 32 (1996) 874-877.

[13] A. Sommerfeld, Electrodynamics. Lectures on theoretical physics. Academic Press (1959).

[14] P. Fernandes and M. Raffetto, Well posedness and finite element approximability of time-harmonic electromagnetic boundary value problems involving bianisotropic materials and metamaterials. Math. Models Methods Appl. Sci. 19 (2009) $2299-2335$.

[15] P. Cocquet, P. Mazet and V. Mouysset, On the existence and uniqueness of a solution for some frequency-dependent partial differential equations coming from the modeling of metamaterials. SIAM J. Math. Anal. 44 (2012) 3806-3833.

[16] T. Kato, Perturbation theory for linear operators, 2nd edition. Springer-Verlag, Berlin (1995).

[17] P. Fernandes, M. Ottonello and M. Raffetto, Regularity of time-harmonic electromagnetic fields in the interior of bianisotropic materials and metamaterials. IMA J. Appl. Math. 79 (2014) 54-93.

[18] P. Monk, Finite element methods for Maxwell's equations. Oxford Science Publications, Oxford (2003).

[19] R.C. Costen and D. Adamson, Three-dimensional derivation of the electrodynamic jump conditions and momentum-energy laws at a moving boundary. Proc. IEEE 53 (1965) 1181-1196.

[20] D. De Zutter, Scattering by a rotating circular cylinder with finite conductivity. IEEE Trans. Antennas Propag. 31 (1983) $166-169$.

[21] C. Hazard and M. Lenoir, On the solution of time-harmonic scattering problems for Maxwell's equations. SIAM J. Math. Anal. 27 (1996) 1597-1630.

[22] A. Alonso and M. Raffetto, Unique solvability for electromagnetic boundary value problems in the presence of partly lossy inhomogeneous anisotropic media and mixed boundary conditions. Math. Models Methods Appl. Sci. 13 (2003) $597-611$.

[23] M.A. Day, The no-slip condition of fluid dynamics. Erkenntnis 33 (1990) 285-296.

[24] T. Shiozawa and I. Kawano, Electromagnetic scattering by an infinitely long cylinder moving along its axis. Electron. Commun. Jpn 53-B (1970) 45-51. 
[25] B.V. Stanić and N.B. Nešković, Electromagnetic reflectivity and scattering by non-uniformly moving plane and cylindrical jet streams. Int. J. Electronics 41 (1976) 351-363.

[26] V. Girault and P.A. Raviart, Finite element methods for Navier-Stokes equations. Springer-Verlag, Berlin (1986).

[27] J.D. Jackson, Classical electrodynamics, 3rd edition. Wiley, New York (1999).

[28] C. Tai, The dyadic Green's function for a moving isotropic medium. IEEE Trans. Antennas Propag. 13 (1965) $322-323$.

[29] J.A. Kong and D.K. Cheng, On guided waves in moving anisotropic media. IEEE Trans. Microwave Theory Tech. 19 (1968) 99-103.

[30] L.J. Du and R.T. Compton Jr., Cutoff phenomena for guided waves in moving media. IEEE Trans. Microwave Theory Tech. 14 (1966) 358-363.

[31] Y. Zhu and S. Granick, Limits of the hydrodynamic no-slip boundary condition. Phys. Rev. Lett. 88 (2002) 106102.

[32] R. Dautray and J. L. Lions, Mathematical analysis and numerical methods for science and technology. Vol. 3: Spectral theory and applications. Springer-Verlag, Berlin (1988).

[33] F. Ben Belgacem, C. Bernardi, M. Costabel and M. Dauge, Un résultat de densité pour les équations de Maxwell, $C$. R. Acad. Sci. Paris Sér. I 324 (1997) 731-736.

[34] J. Jin. The finite element method in electromagnetics. John Wiley \& Sons, New York (1993).

[35] G. Franceschetti, Electromagnetics: theory, techniques and engineering paradigms. Plenum Press, New York (1997).

[36] A.E. Taylor, Introduction to functional analysis. John Wiley \& Sons, New York (1958).

[37] P. Fernandes and M. Raffetto, Existence, uniqueness and finite element approximation of the solution of time-harmonic electromagnetic boundary value problems involving metamaterials. COMPEL 24 (2005) 1450-1469.

[38] R. Dautray and J.L. Lions, Mathematical analysis and numerical methods for science and technology. Vol. 2: Functional and variational methods. Springer-Verlag, Berlin (1988).

[39] M. Raffetto, Ill posed waveguide discontinuity problem involving metamaterials with impedance boundary conditions on the two ports. IET Sci. Measur. Technol. 1 (2007) 232-239.

[40] G. Oliveri and M. Raffetto, A warning about metamaterials for users of frequency-domain numerical simulators. IEEE Trans. Antennas Propag. 56 (2008) 792-798.

[41] P. Fernandes and M. Raffetto, Plain models of very simple waveguide junctions without any solution for very rich sets of excitations. IEEE Trans. Antennas Propag. 58 (2010) 1989-1996.

[42] P. Fernandes and M. Raffetto, Realistic and correct models of impressed sources for time-harmonic electromagnetic boundary value problems involving metamaterials. Int. J. Model. Simul. Sci. Comput. (2013) 1-43.

[43] A.S. Bonnet-Ben Dhia, P. Ciarlet Jr. and C.M. Zwölf, Two- and three-field formulations for wave transmission between media with opposite sign dielectric constants. J. Comput. Appl. Math. 204 (2007) 408-417.

[44] A.S. Bonnet-Ben Dhia, P. Ciarlet Jr. and C.M. Zwölf, Time harmonic wave diffraction problems in materials with sign-shifting coefficients. J. Comput. Appl. Math. 234 (2010) 1912-1919.

[45] S. Caorsi, P. Fernandes and M. Raffetto, On the convergence of Galerkin finite element approximations of electromagnetic eigenproblems. SIAM J. Numer. Anal. 38 (2000): 580-607.

[46] P.G. Ciarlet, Basic error estimates for elliptic problems. Elsevier Science Publishers B. V., Amsterdam, North-Holland (1991).

[47] F. Kikuchi, On a discrete compactness property for the Nedelec finite elements. Journal of the Faculty of Science, University of Tokyo 36 (1989) 479-490.

[48] D. Boffi, Finite element approximation of eigenvalue problems. Acta Numerica (2010) 1-120.

[49] S.H. Christiansen and R. Winther, On variational eigenvalue approximation of semidefinite operators. IMA J. Numer. Anal. 33 (2013) 1-120.

[50] I. Babuska, B. Szabo and I. Katz, The p-version of the finite element method. SIAM J. Numer. Anal. 18 (1981) 515-545.

[51] D. Boffi, Approximation of eigenvalues in mixed form, discrete compactness property, and application to hp mixed finite elements. Comput. Methods Appl. Mech. Eng. 196 (2007) 3672-3681. 